\title{
Theoretical studies of Bose-Hubbard and discrete nonlinear Schrödinger models - Localization, vortices, and quantum-classical correspondence
}

\author{
Peter Jason
}

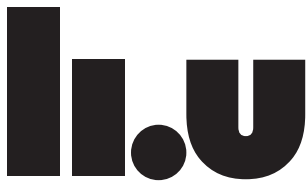

LINKÖPING UNIVERSITY 
ISBN 978-91-7685-735-9

ISSN 0345-7524

Printed by LiU-Tryck, Linköping 2016 
Till minne av min morbror 



\section{Abstract}

This thesis is mainly concerned with theoretical studies of two types of models: quantum mechanical Bose-Hubbard models and (semi-)classical discrete nonlinear Schrödinger (DNLS) models.

Bose-Hubbard models have in the last few decades been widely used to describe Bose-Einstein condensates placed in periodic optical potentials, a hot research topic with promising future applications within quantum computations and quantum simulations. The Bose-Hubbard model, in its simplest form, describes the competition between tunneling of particles between neighboring potential wells ('sites') and their on-site interactions (can be either repulsive or attractive). We will also consider extensions of the basic models, with additional interactions and tunneling processes.

While Bose-Hubbard models describe the behavior of a collection of particles in a lattice, the DNLS description is in terms of a classical field on each site. DNLS models can also be applicable for Bose-Einstein condensates in periodic potentials, but in the limit of many bosons per site, where quantum fluctuations are negligible and a description in terms of average values is valid. The particle interactions of the Bose-Hubbard models become nonlinearities in the DNLS models, so that the DNLS model, in its simplest form, describes a competition between on-site nonlinearity and tunneling to neighboring sites. DNLS models are however also applicable for several other physical systems, most notably for nonlinear waveguide arrays, another rapidly evolving research field.

The research presented in this thesis can be roughly divided into two parts:

1) We have studied certain families of solutions to the DNLS model. First, we have considered charge flipping vortices in DNLS trimers and hexamers. Vortices represent a rotational flow of energy, and a charge flipping vortex is one where the rotational direction (repeatedly) changes. We have found that charge flipping vortices indeed exist in these systems, and that they belong to continuous families of solutions located between two stationary solutions.

Second, we have studied discrete breathers, which are spatially localized and time-periodic solutions, in a DNLS models with the geometry of a ring coupled to an additional, central site. We found under which parameter values these solutions exist, and also studied the properties of their continuous solution families. We found that these families undergo different bifurcations, and that, for example, the discrete breathers which have a peak on one and two (neighboring) sites, respectively, belong to the same family below a critical value of the ring-to-central- 
site coupling, but to separate families for values above it.

2) Since Bose-Hubbard models can be approximated with DNLS models in the limit of a large number of bosons per site, we studied signatures of certain classical solutions and structures of DNLS models in the corresponding Bose-Hubbard models.

These studies have partly focused on quantum lattice compactons. The corresponding classical lattice compactons are solutions to an extended DNLS model, and consist of a cluster of excited sites, with the rest of the sites exactly zero (generally localized solutions have nonzero 'tails'). We find that only one-site classical lattice compactons remain compact for the Bose-Hubbard model, while for several-site classical compactons there are nonzero probabilities to find particles spread out over more sites in the quantum model. We have furthermore studied the dynamics, with emphasize on mobility, of quantum states that correspond to the classical lattice compactons. The main result is that it indeed is possible to see signatures of the classical compactons' good mobility, but that it is then necessary to give the quantum state a 'hard kick' (corresponding to a large phase gradient). Otherwise, the time scales for quantum fluctuations and for the compacton to travel one site become of the same order.

We have also studied the quantum signatures of a certain type of instability (oscillatory) which a specific solution to the DNLS trimer experiences in a parameter regime. We have been able to identify signatures in the quantum energy spectrum, where in the unstable parameter regime the relevant eigenstates undergo many avoided crossings, giving a strong mixing between the eigenstates. We also introduced several measures, which either drop or increase significantly in the regime of instability.

Finally, we have studied quantum signatures of the charge flipping vortices mentioned above, and found several such, for example when considering the correlation of currents between different sites. 


\section{Populärvetenskaplig sammanfattning}

Den här avhandlingen behandlar teoretiska studier av modeller utav främst två typer. Dels studeras så kallade Bose-Hubbard-modeller, vilka är kvantmekaniska modeller som beskriver hur partiklar hoppar (genom kvantmekanisk tunnling) mellan olika potentialbrunnar. Denna typ av modell har blivit väldigt uppmärksammad under de senaste tjugo åren på grund av den snabba utvecklingen inom BoseEinstein-kondensation. Bose-Einstein-kondensat bildas då vissa typer av atomer kyls ner till extremt låga temperaturer, vilket leder till att en stor andel av atomerna hamnar i samma kvanttillstånd. Detta betyder att vissa kvantmekaniska egenskaper som vanligtvis bara är urskiljbara på atomär nivå nu blir makroskopiskt observerbara. Genom att placera ett Bose-Einstein-kondensat i en stående våg som genererats av laserljus, så kommer atomerna i kondensatet antingen att dras till vågens bukar eller noder (beroende på atomslag). Effektivt sett så blir detta en periodisk potential för atomerna, vilket påminner om den som elektronerna i en metall känner av. Bose-Hubbard-modellen beskriver alltså hur de Bose-Einsteinkondenserade atomerna hoppar mellan den stående vågens olika potentialbrunnar (dvs noder eller bukar), men tar också hänsyn till de krafter som finns mellan atomerna själva.

Den andra typen av modell som studeras är av så kallad diskret icke-linjär Schrödinger (DNLS) typ, som beskriver fält som är lokaliserade i brunnarna, istället för partiklar. Denna typ av modell går faktiskt också att applicera på Bose-Einstein-kondensat, då fälten beskriver en form utav medelvärdesbildning av antalet partiklar i brunnarna. Det går att visa matematiskt att Bose-Hubbardmodeller kan approximeras med DNLS-modeller då det är många partiklar i varje brunn. DNLS-modeller är även tillämpbara på många andra typer av system, till exempel kopplade optiska vågledare. Vågledare är, vilket namnet antyder, strukturer som kan leda ljusvågor längs med sig. Ett välbekant exempel på vågledare är optiska fibrer, men det finns även andra typer. Genom att placera flera vågledare nära varandra så kan ljus överföras från en vågledare till en annan, på ett sätt som påminner om hur atomerna i ett Bose-Einstein-kondensat kan hoppa mellan olika brunnar. DNLS-modeller beskriver alltså hur ljuset hoppar mellan olika vågledare, men tar också hänsyn till ljusets (icke-linjära) interaktion med vågledaren själv.

Forskningen som presenteras i denna avhandling kan delas upp i två delar. Dels 
behandlar den nya typer av lösningar till DNLS-modeller. Detta har handlat om virvlar (roterande lösningar) som spontant byter rotationsriktning, men också om lokaliserade lösningar (diskreta 'breathers') i DNLS-modeller där brunnarna har placerats i en ring, med en ytterligare brunn i mitten.

Den andra forskningsdelen har handlat om att studera kopplingen mellan BoseHubbard- och DNLS-modeller. Mer specifikt så har vi letat efter signaturer i BoseHubbard-modeller utav särskilda lösningar och beteenden i motsvarande DNLSmodeller. Detta har dels varit så kallade kompaktoner, för vilka alla brunnar förutom ett fåtal är helt tomma, där vi studerat hur motsvarande kvantmekaniska lösningar ser ut, och även dynamiken för dessa lösningar. Ett annat forskningsprojekt behandlade en viss typ av instabilitet hos en särskild lösning, och vilka kvantsignaturer den lämnar. Vi har även studerat signaturer av de ovannämnda virvlarna, vilka vi studerade i DNLS-modellen. 


\section{Preface}

This dissertation is the result of my doctoral studies carried out from August 2011 to September 2016 in the Theoretical Physics group at the Department of Physics, Chemistry, and Biology (IFM), Linköping University. Certain sections of the thesis are based on my Licentiate thesis from 2014, Comparisons between classical and quantum mechanical nonlinear lattice models.

My research has been focused on theoretical studies of two types of models: discrete nonlinear Schrödinger models and Bose-Hubbard models. The results have been published in peer-reviewed research journals, and are appended to the end of this thesis, with the exception of Paper VI which is included as a manuscript (submitted).

This work was partly supported by the Swedish Research Council. 



\section{Acknowledgements}

I would first and foremost like to thank my supervisor Magnus Johansson! Thank you for these five years, they have been both fun and very rewarding.

My co-supervisor, Irina Yakimenko, who has also tutored me in many of the courses which have been the foundation of my work.

Igor Abrikosov, the head of the Theoretical Physics group. Thank you also for organizing very pleasant and interesting Journal Clubs.

Katarina Kirr, whom I collaborated with on the second paper.

The groups of Belgrade and Santiago, for the pleasant and rewarding meetings in Linköping and Belgrade. A special thanks to Milutin Stepić and co-workers for organizing such a great conference in Belgrade.

Cecilia Goyenola for providing me with the $\mathrm{AT}_{\mathrm{E}} \mathrm{X}$-template for this thesis.

To all (past and present) people in the lunch group for all fun and interesting discussions had over lunch and coffee. Thank you also to everyone involved with the Pub-group.

Till familj, släkt och vänner för allt stöd genom åren. Nu kan ni lära er lite mer om min forskning om bosoner!

Slutligen, till min blivande fru Rebecka. Älskar dig! 



\section{Contents}

1 Introduction 1

1.1 Nonlinear Science . . . . . . . . . . . . . . . . . . . . . . 1

1.2 Nonlinear Models . . . . . . . . . . . . . . . . . . . 2

1.2.1 Discrete Models . . . . . . . . . . . . . . . . . . . 2

1.2 .2 Continuum Models . . . . . . . . . . . . . . . . . 5

1.3 Localization . . . . . . . . . . . . . . . . . 6

1.3.1 Continuous Models . . . . . . . . . . . . . . . . . 6

1.3.2 Discrete Models . . . . . . . . . . . . . . . . . . . . . . . . 9 9

1.3 .3 Compactons. . . . . . . . . . . . . . . . . . . . 10

1.3.4 Lattice Compactons . . . . . . . . . . . . . . . . . . . 11

1.3.5 Linear Localization . . . . . . . . . . . . . . . . . . . 12

1.4 Vortices and Charge Flipping . . . . . . . . . . . . . . . 13

1.5 Chaos and Instabilities . . . . . . . . . . . . . . . . 14

1.5.1 Integrable Models and the KAM Theorem . . . . . . . . . 16

1.5 .2 Stability . . . . . . . . . . . . . . . . 17

1.6 Classical vs Quantum Mechanics . . . . . . . . . . . . . 20

2 Physical Systems 23

2.1 Bose-Einstein Condensates . . . . . . . . . . . . . . . . . 23

2.1.1 Theoretical Treatment . . . . . . . . . . . . . . . . 24

2.1.2 Bose-Einstein Condensates in Optical Lattices . . . . . . . 26

2.2 Optical Waveguide Arrays . . . . . . . . . . . . . . . . 27

2.2 .1 Coupled Mode Theory . . . . . . . . . . . . . . . . . . 31

2.2 .2 Optical Kerr Effect . . . . . . . . . . . . . . . . . . . . 32

2.2 .3 Nonlinear Waveguide Arrays . . . . . . . . . . . 34

3 Discrete Nonlinear Schrödinger Equation $\quad 37$

3.1 General Properties . . . . . . . . . . . . . . . . . 37

$3.1 .1 \quad$ Linear Stability . . . . . . . . . . . . . . . . . . 40

3.2 DNLS and BECs in Optical Lattices . . . . . . . . . . . . . 41

3.3 DNLS and Optical Waveguide Arrays . . . . . . . . . . . 44 
3.4 Extended Models . . . . . . . . . . . . . . . . . . . . . . 45

3.5 Numerical Methods . . . . . . . . . . . . . . . . . . 46

3.6 Instabilities . . . . . . . . . . . . . . . . . . . . . . . . . . . . . . . . . . . .

3.7 Discrete Breathers . . . . . . . . . . . . . . . . . 48

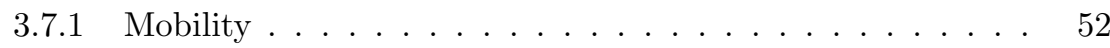

3.7.2 Lattice Compactons . . . . . . . . . . . . . . . 52

3.8 Discrete Vortices . . . . . . . . . . . . . . . . . 53

4 Bose-Hubbard Model $\quad \mathbf{5 7}$

4.1 General Properties . . . . . . . . . . . . . . . . 57

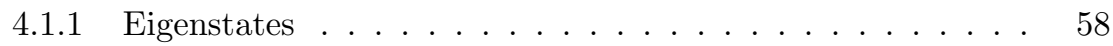

4.2 BECs in Optical Lattices . . . . . . . . . . . . . . . . . . . 60

4.3 Extended Bose-Hubbard Models . . . . . . . . . . . . . 63

4.4 Coherent States . . . . . . . . . . . . . . . . . . . 64

4.5 Connection to the DNLS Model . . . . . . . . . . . . . 67

4.6 Quantum Discrete Breathers .................... 69

4.6.1 Quantum Lattice Compactons . . . . . . . . . . . 72

4.7 Quantum Signatures of Instabilities . . . . . . . . . . . . . . . . . . . . . . . . . . 73

4.8 Quantum Discrete Vortices . . . . . . . . . . . . . . . . 74

4.9 Superfluid to Mott Insulator Transition . . . . . . . . . . . . 76

5 Concluding Comments $\quad 79$

$\begin{array}{lr}\text { Bibliography } & 81\end{array}$

$\begin{array}{lr}\text { List of included Publications } & 97\end{array}$

My contribution to the papers . . . . . . . . . . . . . . 98

$\begin{array}{lr}\text { Related, not included Publications } & 99\end{array}$

$\begin{array}{ll}\text { Paper I } & \mathbf{1 0 1}\end{array}$

Exact localized eigenstates for an extended Bose-Hubbard model with paircorrelated hopping

Paper II

Quantum signatures of an oscillatory instability in the Bose-Hubbard trimer

\section{Paper III}

Quantum dynamics of lattice states with compact support in an extended Bose-Hubbard model

\section{Paper IV}

Charge flipping vortices in the discrete nonlinear Schrödinger trimer and hexamer 
Paper V

Discrete breathers for a discrete nonlinear Schrödinger ring coupled to a central site

\section{Paper VI}

Quantum signatures of charge flipping vortices in the Bose-Hubbard trimer 


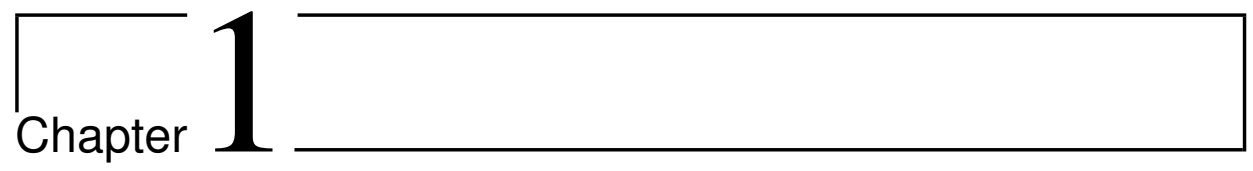

\section{Introduction}

\subsection{Nonlinear Science}

Nonlinear science can be said to be the study of generic phenomena and structures that arise particularly in nonlinear models. It emerged as a unified scientific field in the 1970's, when scientists of different fields realized that the models and phenomena that they were studying also were found in other areas. It thus became of interest to study these models and phenomena in their own right, rather than considering them only within specific contexts. We will see that the models that are encountered in this thesis are applicable to a number of different physical systems. Nonlinear science is thus highly interdisciplinary and relevant to essentially any area of research where mathematical modeling is conducted, ranging from physics, chemistry and biology to meteorology, economics and social sciences [1,2]. Examples of the phenomena of interest include chaos, fractals, turbulence and nonlinear localization.

The term 'nonlinear science' may appear a bit odd and counterintuitive to people unfamiliar with the field. It is generally customary to define a concept by the properties it possesses rather than the ones it lacks, and would it therefore not make more sense to talk about 'linear science' and let nonlinear science be the default? If nothing else, this illustrates the special place linear models have in science. But before we address the question 'what is nonlinear science?' further, let us remind ourselves about what we mean with linearity.

An equation is said to be linear if it obeys the principle of superposition, stating that if $f(\mathbf{r})$ and $g(\mathbf{r})$ are two solutions to the equation, then so are also $C_{1} f(\mathbf{r})+C_{2} g(\mathbf{r})$, where $C_{1}, C_{2}$ are two generally complex constants, and $\mathbf{r}$ the variables of the equation. Consider as an example the classical wave equation

$$
\frac{1}{c^{2}} \frac{\partial^{2} f}{\partial t^{2}}=\frac{\partial^{2} f}{\partial x^{2}}
$$

where $c$ is a constant (the wave's velocity). We can by substitution confirm that the plane wave $f(x, t)=e^{i(q x-\omega t)}$ is a solution, if $\omega / q=c$. There is obviously an infi- 
nite number of $\omega$ and $q$ that satisfy this condition, and all of them result in acceptable solutions. We may now test that also $f(x, t)=C_{1} e^{i\left(q_{1} x-\omega_{1} t\right)}+C_{2} e^{i\left(q_{2} x-\omega_{2} t\right)}$, and even $f(x, t)=\sum_{j} C_{j} e^{i\left(q_{j} x-\omega_{j} t\right)}$, are solutions if $\omega_{j} / q_{j}=c$, confirming that Eq. (1.1) indeed is linear. Equation (1.1) is linear because derivatives (of any order) are linear operators

$$
\frac{\partial\left(C_{1} f(x)+C_{2} g(x)\right)}{\partial x}=C_{1} \frac{\partial f(x)}{\partial x}+C_{2} \frac{\partial g(x)}{\partial x} .
$$

A nonlinear equation is consequently one for which the principle of superposition does not hold. Why is this important, you might ask. Superpositions enable us to reduce the solution into simpler constituents that can be analyzed separately, which computationally is a big advantage. Consider again Eq. (1.1), which we in principle solved when we concluded that $e^{i(q x-\omega t)}$ is a solution. Physically relevant functions can be decomposed into plane waves, meaning that the time evolution of the initial conditions are entirely determined by that of the individual plane waves. For a nonlinear equation we would have to consider the full problem as a whole, which generally makes it much more difficult to solve. Another viewpoint is that a nonlinearity in Eq. (1.1) introduces interactions between the plane waves. This raises interesting philosophical questions regarding for instance causality (the interested reader is directed to [1]).

The rest of this chapter contains a rather general overview of certain models and concepts connected to nonlinear science, but for a more complete review and historical account, the reader is directed to the books by Scott $[1,3]$. Note also that the main models of this thesis, the discrete nonlinear Schrödinger (DNLS) and Bose-Hubbard model, are not covered in this chapter but have chapters 3 and 4 , respectively, dedicated to them. We will also discuss in these chapters how the concepts introduced in the current chapter apply to these particular models.

\subsection{Nonlinear Models}

When modeling a dynamical process, time $^{1}$ may be treated either as a discrete or a continuous variable. In the former case, we usually call the model a mapping, which we do not consider much in this thesis (only briefly when discussing the stability of periodic solutions), and the interested reader is directed to the vast literature on nonlinear and dynamical systems $[2,4]$. Models that treat time as a continuous variable may be further categorized according to whether the dynamical variables of the system form a discrete, numerable set or a continuum.

\subsubsection{Discrete Models}

The discrete models that we are concerned with consist of coupled ordinary differential equations. We will furthermore primarily focus on models where all the

\footnotetext{
${ }^{1}$ The role of time is in some cases taken by another physical quantity. For example, in optical waveguide systems (Secs. 2.2 and 3.3) we are generally interested in how the light changes with the propagation length into the waveguide (this is equivalent to the propagation time if you 'follow' the light).
} 
dynamical variables represent the same physical quantity, which are repeated in some sort of periodic structure. These models may either be connected to physical systems that are fundamentally discrete, e.g. the ions in a metal, or they may arise due to a tight-binding treatment on a continuous system. Examples of the latter, which are considered in this thesis, are electromagnetic fields in optical waveguides (Secs. 2.2 and 3.3) and the (average) number of Bose-Einstein condensed atoms in optical lattice potential wells (Secs. 2.1 and 3.2).

To illustrate how nonlinearities can enter a discrete model, consider the case of a one-dimensional chain of equal atoms connected with linear springs, for which the equations of motion are given by

$$
m \ddot{x}_{j}=K\left(x_{j+1}-2 x_{j}+x_{j-1}\right)
$$

where $x_{j}$ is the displacement of the $j$-th atom from its equilibrium position, $K$ the spring constant, $m$ the atomic mass and dots indicate temporal derivatives. Let us further assume that the chain has periodic boundary conditions, also containing in total $N$ atoms. This is solved by a plane wave ansatz $x_{j}=A e^{i(q j-\omega t)}$, giving the phonon spectrum familiar from elementary solid state physics [5]

$$
\omega= \pm 2 \sqrt{\frac{K}{m}}|\sin (q / 2)|
$$

where $q=2 \pi k / N, k \in \mathbb{Z}$. All the different atomic vibrations in this linear chain are thus expressible as linear combinations of these normal modes. As long as the springs are linear and there are no additional external forces, the model remains linear. It can thus be modified in many different ways while preserving the linearity, for instance by introducing longer ranged interactions, using a basis with more than one type of atom, or even breaking the translational symmetry by introducing vacancies, impurities or disorder. The Hamiltonian (energy) associated with Eq. (1.3) is

$$
H=\sum_{j}\left[\frac{p_{j}^{2}}{2 m}+\frac{K}{2}\left(x_{j+1}-x_{j}\right)^{2}\right],
$$

where $p_{j}=m \dot{x}_{j}$ is the momentum of the $j$-th atom and

$$
U=\sum_{j} \frac{K}{2}\left(x_{j+1}-x_{j}\right)^{2}
$$

is the potential energy. This potential is often the lowest order expansion of a more realistic nonlinear inter-atomic potential, and thus strictly valid only for small oscillations. A natural extension of the model is simply to include more terms of the expansion, which consequently make the associated equations of motion nonlinear. In 1953, Enrico Fermi, John Pasta, and Stan Ulam did just this, and considered a mass-spring model with the three lowest-order terms,

$$
U=\sum_{j}\left[\frac{K}{2}\left(x_{j+1}-x_{j}\right)^{2}+\frac{\alpha}{3}\left(x_{j+1}-x_{j}\right)^{3}+\frac{\beta}{4}\left(x_{j+1}-x_{j}\right)^{4}\right],
$$


a model that nowadays is called the Fermi-Pasta-Ulam (FPU) lattice. They used it to numerically study the thermalization of energy in a crystal, and in contrast to their expectation that the energy would spread out over the normal modes, they observed that the initial condition seemed to recur periodically in the dynamics, in apparent disagreement with statistical mechanics [6]. The FPU-problem, as it is called, has since been widely debated and is an active field of research [7]. The work by Fermi, Pasta, and Ulam is also notable because it was one of the earliest applications of a computer to a physical problem, using a vacuum tube computer called MANIAC ${ }^{2}$.

Discrete models can also contain anharmonic on-site potentials,

$$
U=\sum_{j} U_{j}\left(x_{j}\right)
$$

This type of potential arises naturally when modeling different kinds of oscillators, for example atomic bonds in molecules, but may also be due to externally applied forces. In the case of BECs in optical lattices, it is the inter-atomic interactions that give rise to the on-site potential, whereas for optical waveguides it comes from an interaction between the light and the medium it propagates in. The model obtained by adding an anharmonic on-site potential to the potential (1.6) of the linear mass-spring system (1.3) is called the discrete nonlinear Klein-Gordon model, the equations of motion given by

$$
m \ddot{x}_{j}=K\left(x_{j+1}-2 x_{j}+x_{j-1}\right)-\frac{\partial U_{j}\left(x_{j}\right)}{\partial x_{j}} .
$$

It is called so because in an appropriate continuum limit, the model takes the form of the Klein-Gordon equation with some additional nonlinear terms. To see this, we introduce the variable $y(j d)=x_{j}$, where $d$ is the inter-atomic spacing. Equation (1.9) can then be written as

$$
\ddot{y}(j d)=\frac{K d^{2}}{m} \frac{y((j+1) d)-2 y(j d)+y((j-1) d)}{d^{2}}-\frac{1}{m} \frac{\partial U_{j}(y(j d))}{\partial y(j d)} .
$$

If $d$ is very small, and both $y(j d)$ and $f(y(j d))=m^{-1} \partial U_{j}(y(j d)) / \partial y(j d)$ vary slowly with $j$, the first term on the right-hand side approaches a second order derivative, giving

$$
\ddot{y}(r)=\mathbb{C} \frac{\partial^{2} y}{\partial r^{2}}-f(y(r))
$$

with $\mathbb{C}=K d^{2} / m$, and $r=j d$ now is allowed to take any value. This has the same form as the relativistic Klein-Gordon equation with a nonlinear correction $f(y(r))$. It is of course not a unique property of the discrete nonlinear Klein-Gordon lattice to be well approximated by a continuum model in certain limits. Let us also point out that dynamical variables not necessarily are real, where for example the DNLS equation (chapter 3) has complex variables.

\footnotetext{
${ }^{2}$ Mathematical Analyzer, Numerator, Integrator, and Computer.
} 
As mentioned in connection with the FPU-lattice, models often become essentially linear in the small-amplitude limit. One can thus, in some cases at least, control the influence of nonlinear effects in experiments by adjusting the amplitude of the excitation. This can also be used to confirm that an observation indeed is caused by nonlinear effects. For some systems it would however be quite misleading to say that they become linear in a small-amplitude limit, since it can be very challenging to produce any nonlinear response experimentally (an example being for optical waveguides, discussed in Sec. 2.2, where generation of nonlinear effects requires high-intensity lasers).

\subsubsection{Continuum Models}

We now turn to continuum models, which generally consist of partial differential equations that describe the evolution of different types of fields. The first one we tackle is the nonlinear Schrödinger (NLS) equation ${ }^{3}$

$$
i \frac{\partial \psi(x, t)}{\partial t}+\frac{\partial^{2} \psi(x, t)}{\partial x^{2}} \pm 2|\psi(x, t)|^{2} \psi(x, t)=0
$$

called so due to its similarity with the quantum mechanical wave equation (it does not necessarily describe a quantum system though). This is also, as the name implies, the continuum limit of the DNLS model studied in chapter 3. The imaginary number $i$ in the equation indicates, which readers familiar with quantum mechanics already know, that the field $\psi(x, t)$ is a complex function.

There are several different versions of Eq. (1.12) that also are referred to as NLS equations, for instance in higher dimensions, $\psi$ being a multi-component vector, or with other types of nonlinearities. When necessary to be more specific, Eq. (1.12) can be called the single-component one-dimensional NLS equation with cubic nonlinearity. Equation (1.12) however has a special property that most other versions lack, namely that it is integrable ${ }^{4}[3]$. For continuum models, integrability means that the equation has an infinite number of conserved quantities. This property is intimately connected to the existence of a special type of localized solutions called solitons, which we will return to in Sec. 1.3.

One reason for the NLS equation's importance and fame is that it is a generic equation that arises when one considers a wave packet with lowest order contributions from dispersion and nonlinearity. It therefore has applications in several different areas, including nonlinear optics, nonlinear acoustics, deep water waves and plasma waves [3]. It has also played a prominent role for Bose-Einstein condensates, usually also containing a potential $V(x)$ due to external electromagnetic fields, but is in this context often referred to as the Gross-Pitaevskii equation [9] (see Sec. 2.1.1).

Let us consider two more famous and important integrable nonlinear equations,

\footnotetext{
${ }^{3}$ All equations considered in this section are given in normalized units.

${ }^{4}$ There exist other NLS-type equations that are integrable, for example one with nonlocal nonlinearity [8], but it is an exceptional property.
} 
the first one being the Korteweg-de Vries (KdV) equation

$$
\frac{\partial u}{\partial t}+\frac{\partial^{3} u}{\partial x^{3}}-6 u \frac{\partial u}{\partial x}=0
$$

where $u(x, t)$ is a real function. This is arguably the most important soliton equation, and it is named after two Dutch physicists, Diederik Korteweg and Gustav de Vries, who in 1895 used it to explain the occurrence of localized waves in water [10]. Like the NLS equation, the $\mathrm{KdV}$ is a generic equation, and it arises when considering long waves in a dispersive medium with lowest order nonlinearity. The applications are thus also numerous and include, apart from water waves, ion-acoustic waves in plasma, pressure waves, and the rotational flow of a liquid through a tube [3].

The final continuous model we consider is the Sine-Gordon (SG) equation

$$
\frac{\partial^{2} u}{\partial x^{2}}-\frac{\partial^{2} u}{\partial t^{2}}=\sin (u)
$$

This is a special case of the nonlinear Klein-Gordon equation (1.11) with $f(y)=$ $\sin (y)$. Interestingly, the SG equation is also Lorentz invariant and its solutions possess certain properties usually associated with special relativity, for example length contraction (the solutions contract when they move faster). The SineGordon equation has for example been used to describe the dynamics of crystal defects and domain walls in ferromagnetic and ferroelectric materials, and the propagation of quantum units of magnetic flux in Josephson junctions [3].

\subsection{Localization}

\subsubsection{Continuous Models}

The study of nonlinear localization can be dated back to 1834, when Scottish naval engineer John Scott Russell observed what he called a 'Wave of Translation' in the Union Canal near Edinburgh. Russell was conducting experiments with boats in the canal when he saw [11]

... a large solitary elevation, a rounded, smooth and well-defined heap of water, which continued its course along the channel without change of form or diminution of speed.

Russell would continue to study this type of solitary waves, and was able to deduce a number of interesting properties. His observations were however met with big skepticism from some of the leading scientists at the time since the current linear models for shallow water did not permit such solutions. This disagreement is rather characteristic for nonlinear science, in that the discoveries of many phenomena have come as surprises to the scientific community, and often also been met with skepticism, in part at least because the new discoveries contradict the intuition based upon more familiar linear models. Other scientists did however support Russell in this question, and independently confirmed his observations. Eventually 
Korteweg and de Vries derived their eponymous equation (1.13) for shallow water waves, and showed that it indeed supports solutions of the type described by Russell [10]. The work of Korteweg and de Vries did however not initiate much further work on nonlinear localization at the time, so we turn the clock forward about half a century to Enrico Fermi, John Pasta and Stan Ulam's study of the FPU lattice (1.7). To solve the mystery of the recurring initial conditions in the anharmonic chain, in 1965 Norman Zabusky and Martin Kruskal [12] approximated the FPU lattice with the KdV equation (1.13), and found that the initial condition would split up into several solitary waves that reassembled in approximately the original state after some time. The solitary waves also showed peculiar properties when they collided: they emerged from collisions with essentially the same shape and velocity as they had prior to it, but slightly shifted compared to the position they would have had if there were no collision. This behavior reminded Zabusky and Kruskal of colliding particles, so they named these solitary waves solitons.

Let us emphasize that the solitons' invariance under collisions is not what one would have expected. The KdV equation (1.13) is nonlinear, and the individual waves are therefore interacting with each other when they overlap. One would rather expect that a collision would have a big effect on (at least) the shape and velocity of the waves. The shift also indicates that the mechanism here is something fundamentally different from the linear superposition. The invariance under collisions is thought to be connected to the integrability of the equations that support them [13]. We should also note that there is not a single, universally agreed on definition of a soliton, and that this can vary from very strict mathematical definitions to being essentially synonymous to a solitary wave, which often is the case in the physics community.

Can we have some intuitive understanding of why solitons exist in certain continuous nonlinear models? One may consider them to essentially be the result of balancing the linear dispersion of a wave with the contraction from the nonlinearity. To illustrate the former factor, consider the linear part of the KdV equation (1.13)

$$
\frac{\partial u}{\partial t}+\frac{\partial^{3} u}{\partial x^{3}}=0
$$

This is, due to the linearity, solved by any superposition of the exponential functions $e^{i(q x-\omega(q) t)}$, with $\omega(q)=-q^{3}$. The time-evolution for an arbitrary initial wave profile, $u(x, t=0)=f(x)$, is therefore determined by

$$
u(x, t)=\frac{1}{2 \pi} \int_{-\infty}^{\infty} F(q) e^{i(q x-\omega(q) t)} d q
$$

where

$$
F(q)=\int_{-\infty}^{\infty} f(x) e^{-i q x} d x
$$



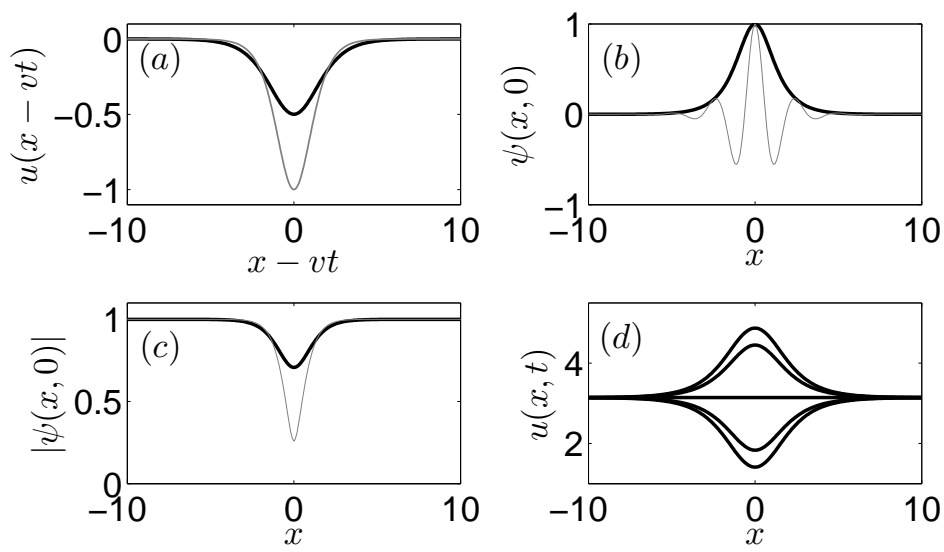

Figure 1.1. (a) Soliton (1.20) for the KdV equation, with $v=1$ (thick black) and $v=2$ (thin gray). (b) Bright soliton (1.21) for the NLS equation, with $a=1, v_{e}=5$ and $t=0$. The thick, solid black line indicate $|\psi(x, 0)|$ and the solid gray $\operatorname{Re}(\psi(x, 0))$. (c) $|\psi(x, 0)|$ for the dark soliton (1.22) of the NLS equation with a minus sign at $t=0$, with $a=1$, and $\theta=\pi / 4$ (thick black), $\theta=\pi / 12$ (thin gray). (d) Breather (1.23) of the SG equation, for $\beta=\omega=1 / \sqrt{2}$, with $t$ going from $\pi / 2 \omega$ (top) to $3 \pi / 2 \omega$ (bottom) in increments of $\pi / 4 \omega$.

is the Fourier transform of $f(x)$. Should $f(x)$ now be spatially localized, then it must contain significant contributions from a wide range of Fourier modes ${ }^{5}$, each of which traveling with a different phase velocity $v_{p}=\omega / q=-q^{2}$. This will consequently cause the wave to disperse, which is also the reason why the dependence of $\omega$ on $q$ is called the dispersion relation.

Consider now instead the effect of the nonlinear term in the $\mathrm{KdV}$ equation

$$
\frac{\partial u}{\partial t}-6 u \frac{\partial u}{\partial x}=0
$$

Plugging in a traveling wave ansatz, $u(x, t)=f(x-v t)=f(\chi)$ leads to

$$
-[v+6 f(\chi)] f^{\prime}(\chi)=0,
$$

which suggests that waves with larger amplitude move faster. For an initially localized wave, this means that the parts with large amplitude will 'catch up' with those in front with lower amplitude, resulting in a steepening of the wave.

For the full $\mathrm{KdV}$ equation, these two effects can be balanced to create a stable, localized wave. It is readily tested that Eq. (1.13) is satisfied by

$$
u(x, t)=-\frac{v}{2} \operatorname{sech}^{2}\left(\frac{\sqrt{v}}{2}(x-v t)\right)
$$

\footnotetext{
${ }^{5}$ This is related to Heisenberg's uncertainty principle from quantum mechanics, which states that a narrow wave-packet in real space implies a broad wave-packet in momentum (Fourier) space.
} 
which indeed is a soliton ${ }^{6}$, traveling with velocity $0<v<\infty$. Examples of this soliton, with $v=1$ and $v=2$, are shown in Fig. 1.1(a).

Also the NLS equation (1.12) supports solitons, but of different types depending on the sign in the equation. Bright solitons are supported for a plus sign in (1.12), which are localized density elevations, for instance of the type [3]

$$
\psi(x, t)=a \exp \left(\left[i \frac{v_{e}}{2} x+i\left(a^{2}-\frac{v_{e}^{2}}{4}\right) t\right]\right) \operatorname{sech}\left[a\left(x-v_{e} t\right)\right]
$$

where the amplitude $a$ and the envelope velocity $v_{e}$ are independent. Unlike the $\mathrm{KdV}$-soliton, this is a complex-valued function, consisting of a localized envelope modulated by a plane wave. The NLS equation (1.12) with minus sign instead supports dark solitons, which are localized density dips, an example being [3]

$$
\psi(x, t)=a \exp \left(2 i a^{2} t\right)\{(\cos \theta) \tanh [a(\cos \theta)(x-v t)]-i \sin \theta\},
$$

where $a$ now is the amplitude of the background, $-\pi / 2 \leq \theta \leq \pi / 2$ determines the depth of the dip, and $v=a \sin \theta$ is the velocity. Figs. $1.1(\mathrm{~b})$ and $1.1(\mathrm{c})$ show examples of the bright and dark solitons, respectively.

The NLS equation and SG equation support localized solutions, which also are time periodic, called breathers. The NLS breather consists of a two-soliton bound state [14], while the Sine-Gordon breather has the form [3]

$$
u(x, t)=4 \arctan \left(\frac{\beta \sin (\omega t)}{\omega \cosh (\beta x)}\right)
$$

with $\omega^{2}+\beta^{2}=1$, shown in Fig. 1.1(d).

\subsubsection{Discrete Models}

Localized structures can also exist in nonlinear lattice models with translational symmetry. There exist integrable discrete equations which possess exact discrete soliton solutions, two famous examples being the Toda lattice and the AblowitzLadik model ${ }^{7}[3]$.

Another example of localized solutions is discrete breathers (DBs), also called intrinsic localized modes (ILMs), which are not only localized but also timeperiodic (breathing) $[15,16]$. An intuitive example of a DB would be in a (translationally invariant) anharmonic mass-spring system, where only one (or a few) of the masses oscillates significantly (the amplitude of oscillations may for instance decay exponentially from this point) [16].

A central paper in this field is due to MacKay and Aubry in 1994 [17], where the existence of DBs in anharmonic Hamiltonian systems with time-reversibility was rigorously proven under rather general conditions, thus showing that DBs

\footnotetext{
${ }^{6}$ One may argue that this actually is not a soliton, but rather a solitary wave, since it does not have another soliton to collide with.

${ }^{7}$ The Ablowitz-Ladik model is rather similar to the DNLS model, with the nonlinear term $\left|\psi_{j}\right|^{2} \psi_{j}$ in Eq. (3.2) replaced with $\left|\psi_{j}\right|^{2}\left(\psi_{j-1}+\psi_{j+1}\right)$. This model will also converge to the NLS equation in the continuum limit.
} 
are generic entities. This work has also been extended to more general systems [18]. The crucial point is that a non-resonance condition is fulfilled, i.e. that all multiples of the frequency of the DB fall outside the bands of the linear modes. The discreteness is essential for this, as it bounds the frequency of the linear modes (cf. the optical and acoustic branches of phonons). Compare this to a continuous, spatially homogeneous model, where the linear spectrum is unbounded, and there surely is at least some multiple of the frequency of the continuous breather that falls in a linear band. There are some notable exceptions for integrable equations which we encountered in the previous Sec. 1.3.1. The nonlinearity on the other hand enables the frequency of the DB to fall outside the linear bands.

One interesting aspect of the proof in [17] is that it also provides an explicit method for constructing DBs [19]. It starts from the so called anti-continuous limit where all sites are decoupled from each other, and one trivially can create a localized solution, let us say on one site, simply by setting this site into motion and letting all others be still. The key idea of the method, and thus also the proof, is that when the coupling between the sites is turned up slightly, the 'old' localized solution of the uncoupled model can be mapped on a 'new' localized solution of the coupled model, if the above mentioned condition is fulfilled. This new solution can in practice be found by using the old solution as the initial guess in a Newton-Raphson algorithm (see Sec. 3.5 for the application to the DNLS model). By iterating this procedure, i.e. turning up the coupling and finding a new localized solution, one can follow a family of discrete breathers as a function of the coupling [16]. Depending on which solution one starts with in the anticontinuous limit, different discrete breather families may be followed.

DBs have been thoroughly studied theoretically with many different models (see e.g. [16] and references therein, and Sec. 3.7 for DBs in DNLS models), but also experimentally observed in a wide variety of systems such as Josephson junctions [20-22], crystal lattice vibrations [23], antiferromagnetic structures [24,25], micromechanical cantilevers [26-28], and coupled pendulums [29,30], as well as optical waveguide arrays and BECs in optical lattices which will be discussed in more depth in Secs. 2.1.2 and 2.2.3.

\subsubsection{Compactons}

Localized solutions generally have nonzero tails that decay (typically exponentially) when moving away from the solution's core. This means that even though two solitons are far apart, there is still some interaction between them. There are however certain nonlinear models that do support localized solutions that indeed become exactly zero outside a given region. The finite interaction range of such solutions make them interesting for practical applications in e.g. information transmission. In mathematical jargon, these solutions have a compact support, and are therefore called compactons. The concept of a compacton was introduced in a paper by Rosenau and Hyman, for a class of generalized KdV equations with nonlinear dispersion [31]

$$
\frac{\partial u}{\partial t}+\frac{\partial u^{m}}{\partial x}+\frac{\partial^{3} u^{n}}{\partial x^{3}}=0
$$



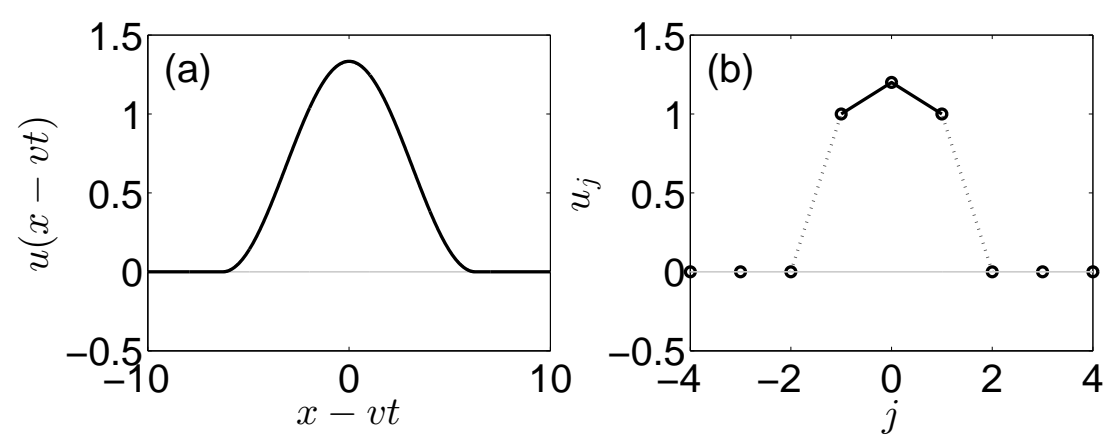

Figure 1.2. (a) Compacton (1.25) with $v=1$. (b) Illustration of a three-site lattice compacton. Dotted lines indicate that the edge sites are decoupled from the empty lattice (assuming only nearest neighbor interactions).

with $m>0$ and $1<n \leq 3$. For $m=2, n=1$, the original (rescaled) $\mathrm{KdV}$ equation is recovered, but their main focus was on the equation with $m=n=2$ (the Rosenau-Hyman equation), which supports compactons of the form [31]

$$
u(x, t)=u(x-v t)= \begin{cases}\frac{4 v}{3} \cos ^{2}[(x-v t) / 4] & \text { if }|x-v t| \leq 2 \pi \\ 0 & \text { otherwise, }\end{cases}
$$

shown in Fig. 1.2(a). The compacton has a discontinuity in its second derivative at the compacton boundary $|x-v t|=2 \pi$, but since the spatial derivatives in Eq. (1.24) are taken on $u^{2}$, this will not cause any problems. Even though the equation is not integrable ${ }^{8}$, these compactons remarkably show properties similar to solitons in collisions, since there is no radiation produced in the collision, but only some compacton-anticompacton pairs (justifying compacton). Compactons were also obtained for other $m$ and $n$ in [31], but not for the original KdV however. In fact, compactons cannot exist in continuous models with linear dispersion, as this would cause a spreading at the low amplitude edges which destroys the compactness.

\subsubsection{Lattice Compactons}

Just like with continuous solitons, discrete breathers generally have exponentially decaying tails, and thus an infinite span. For discrete models, a lattice compacton consists of a cluster of excited sites, while the rest of the lattice has exactly zero amplitude (Fig. 1.2(b) illustrates a 3-site compacton). The basic idea to obtain lattice compactons is to completely decouple the compacton from the rest of the lattice. In the commonly studied case of only nearest-neighbor interactions, this means that the couplings between the sites at the edge of the compacton and their empty neighbor effectively are zero (illustrated with dotted lines in Fig. $1.2(\mathrm{~b}))$. Refs. $[32,33]$ utilized this property to derive conditions, for certain types

\footnotetext{
${ }^{8}$ Equation (1.24) has only four known conserved quantities [31].
} 

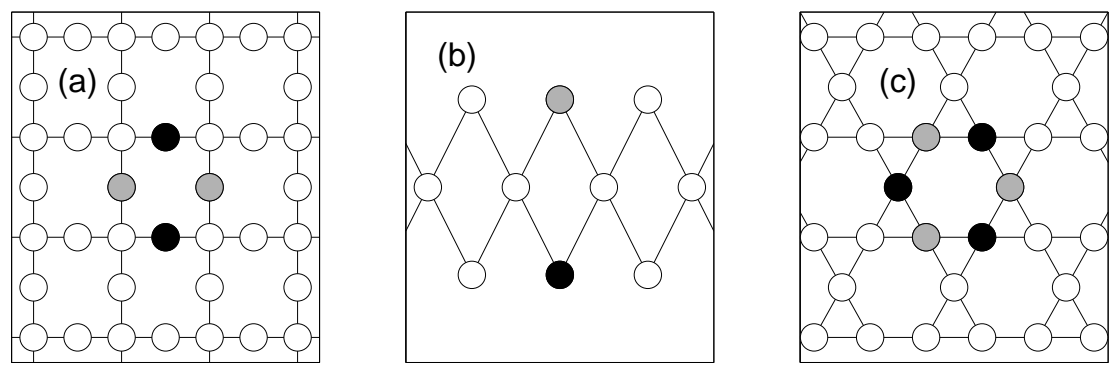

Figure 1.3. Compact modes in linear lattices with flat bands: (a) Lieb lattice, (b) diamond chain, (c) kagomé. The lattices have isotropic nearest-neighbor coupling which is indicated with lines. White circles represent empty sites, while the black and gray sites have equal amplitude but are out of phase with each other, which results in destructive interference at the empty neighbor that decouples the compact modes from the rest of the lattice.

of models, for lattice compactons to be possible, and could also explicitly construct models that possess compactons (and also studied these). For instance, it turns out that compactons are not allowed for the ordinary DNLS equation, but however for different extensions of the model [32]. Most notably for this thesis, lattice compactons exist in an extended DNLS model derived for optical waveguide arrays [34] (see Secs. 3.4 and 3.7.2), which we studied the quantum analogs of in Papers I and III (see also Sec. 4.6.1). More recently it was shown that compactons also can exist in a DNLS model with a fast, periodic modulation of the nonlinearity, both in one- [35] and several-dimensional [36] lattices. By time averaging over the fast modulation, the (nearest-neighbor) coupling becomes density dependent, and it will also vanish for certain amplitudes which allow for compactons.

The lattice compactons discussed in [32-34] are fundamentally discrete entities, and will not correspond to continuous compactons in a continuum limit [37] (the jump in amplitude at the lattice compacton edges contradicts the assumption of slowly varying amplitudes). Generally, discretizing a continuous compacton does not lead to a lattice compacton, but rather to a solution which decays with superexponential tails [38-40]. The discretized compacton can however follow its continuous counterpart very well, even when it is localized only on a few sites and thus far from the continuum limit [41].

\subsubsection{Linear Localization}

Localization is in no way restricted to nonlinear models, but can also occur in linear ones. With the discussion in Sec. 1.3.1 in mind, we realize that localized waves can exist in continuous (homogenous) linear models if the dispersion relation is linear, meaning that all plane waves travel at the same speed. A familiar example of this is light propagating in vacuum, where the fact that all wavelengths of light travel 
at same speed is a cornerstone of modern physics. Localization in dispersive linear systems is usually associated with a broken (continuous or discrete) translational symmetry, due to for instance impurities, vacancies or disorder (Anderson localization). There are however certain linear, periodic models that actually support compact solutions, namely those that possess flat bands [42]. This is generally an effect of the lattice geometry, where for compact solutions the coupling between occupied and empty sites are canceled due to destructive interference. This is illustrated for some well known flat-band lattice geometries in Fig. 1.3.

\subsection{Vortices and Charge Flipping}

Vortices are ubiquitous structures that arise in a wide variety of physical systems, ranging from the hydrodynamic vortices seen in bathtubs and kitchen sinks at home to violent tornados, but they are also present in more exotic systems like superconductors and superfluids. For systems that are described by fields with both amplitude and phase, for example electromagnetic fields or the matter field of a BEC, the energy flow is generally related to the gradient of the field's phase. A vortex is in this case related to a phase singularity (the vortex core), where the amplitude of the field vanishes and around which the phase changes by a multiple of $2 \pi$ (see Fig. 1.4(a)). This multiple is often called the vortex' topological charge (also called vorticity or winding number), and its sign indicates the direction of the rotational flow.

For fields governed by nonlinear equations it is under certain circumstances possible to obtain vortex solitons [43], where the amplitude of the field is localized around the vortex core. If the field furthermore is embedded in a lattice structure, examples being BECs in optical lattices (Sec. 2.1.2) and optical waveguide arrays (Sec. 2.2.3), then lattice vortex solitons or discrete vortex solitons may be obtained. The field is then mainly confined to a few lattice wells that are surrounding the vortex core. Discrete vortex solitons have been studied with continuous models [44-46], but this is also a situation where discrete models may be valid, and also have been used [46-55] (see also Sec. 3.8). For discrete models, the topological charge for a closed loop of sites (or for the cell enclosed by the loop) can be defined in a corresponding way as the accumulated phase (in $2 \pi$-units) when following the loop:

$$
T C=\frac{1}{2 \pi} \sum_{<\mathbf{q}, \mathbf{r}>} \arg \left(\psi_{\mathbf{q}}^{*} \psi_{\mathbf{r}}\right)
$$

where $\psi_{\mathbf{r}}$ is the complex field at the site labeled with $\mathbf{r}$, and the sum runs over neighboring sites along the given loop (cf. Fig. 1.4(b)-(c)). By restricting the phase differences between two sites to $]-\pi, \pi]$ the topological charge becomes restricted to integer values in the interval $-f / 2<T C \leq f / 2$, where $f$ is the number of sites in the loop. Vortices can also be studied in quasi-one dimensional systems such as lattice rings [53-55], where the vortex is related to the net flow in the ring (Fig. $1.4(\mathrm{c})$ ). Such a system may be treated as a one-dimensional periodic lattice (if one uses a discrete model). 

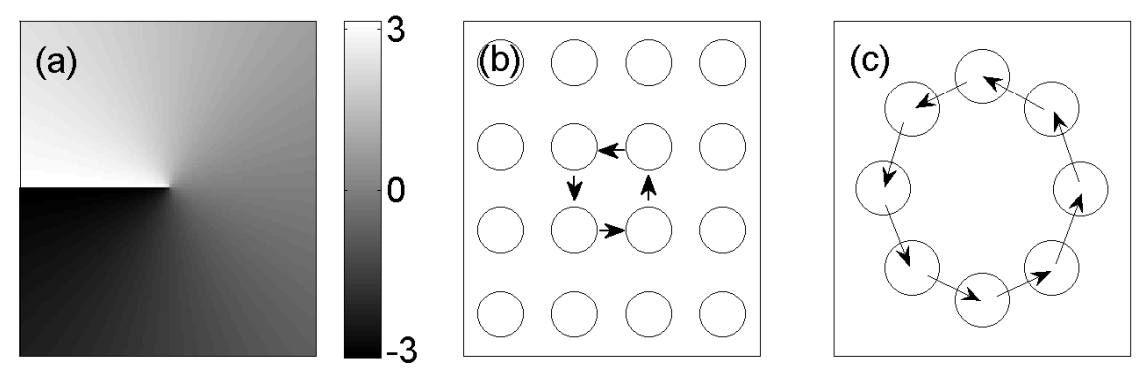

Figure 1.4. (a) The phase of a vortex, which changes by a multiple of $2 \pi$ around the phase singularity (in this case one). (b) In a discrete system, the topological charge is calculated by adding up the phase difference between adjacent sites along a closed loop, marked with arrows in the plot (the circles represent the sites). (c) Vortices can also occur in quasi-one dimensional systems, such as rings, where a vortex inside the circle generates a net flow around the ring.

For discrete models that arise due to a discretization of an underlying continuous field, the (discrete) topological charge indicates the total topological charge of the continuous field vortices which are located in the region enclosed by the closed loop. It may thus be of interest to get information about the underlying field ${ }^{9}$, and several works $[53-56]$ with discrete models have therefore interpolated of the field between the sites, for instance to gain information about where the vortex cores are located, how many they are, and how they interact.

The topological charge is a conserved quantity in systems with continuous rotational symmetry around the vortex core, but by breaking the symmetry its value is allowed to change. One way to break this symmetry is to introduce a lattice, in which case the vortex exchanges angular momentum with the lattice when the topological charge changes. It is also possible to obtain vortices that repeatedly change the sign of the topological charge, and thus also the direction of the flow, which are therefore called charge flipping vortices.

Since the topological charge is restricted to integer values, vortices could potentially be used to store information [57], similar to a bit in a computer. A detailed understanding of the charge flipping could therefore be of great importance for realizing for example logical circuits. In paper III we study charge flipping vortices in DNLS trimers and hexamers, i.e. three- and six-site quasi-one dimensional lattices of the type illustrated in Fig. 1.4(c) (also discussed in Sec. 3.8).

\subsection{Chaos and Instabilities}

The most famous nonlinear phenomenon, at least for the public audience, is probably chaos. This can even be considered as part of our popular culture today,

\footnotetext{
${ }^{9}$ To obtain the actual field one obviously needs to solve the continuous problem.
} 
where there even are popular fictional movies and books dealing with it. We will only give a very brief description of this large area, so the interested reader is directed to any of the numerous text books on the subject, e.g. [2, 4, 13, 58].

Chaotic systems are characterized by their sensitive dependence of initial conditions (SIC), meaning that even minute changes of the initial conditions may cause a drastic change in the behavior of the system. This implies that, since the configuration of a system only can be determined up to a certain accuracy, the system in practice is unpredictable after some time ('Lyapunov time'), even though the time evolution is governed by completely deterministic equations. This is commonly referred to as the 'butterfly effect', a term coined by the American mathematician and meteorologist Edward Lorenz, who pioneered chaos theory. Lorenz famously gave a talk entitled 'Does the flap of a butterfly's wings in Brazil set off a tornado in Texas?' at a scientific conference in 1972 which in a dramatic way encapsulates the essence of the butterfly effect: very small changes in the initial conditions can over time have enormous, and possibly disastrous, effects on the outcome ${ }^{10}$. It would of course not have come as a surprise to discover that extremely complicated models with millions of degrees of freedom show a very complex behavior, but a crucial point of chaos theory is that this also occurs for fairly simple (but nonlinear) and low dimensional models. Lorenz himself studied a three-dimensional nonlinear model (now called the Lorenz model) for the weather, when he discovered chaos. The discovery happened almost by accident, as he rounded off the numerical value of an initial condition in his simulations and noticed that the outcome changed drastically. Similar to the case with solitons, the discovery of chaos came in conflict with some of the (linearly based) beliefs held at the time, namely that the evolution of two closely lying initial conditions will be similar.

To define chaos more precisely, let us assume that we have a system with $N$ state variables, so that the state of the system at time $t$ can be denoted as $\mathbf{x}(t)=\left(x_{1}(t), \ldots, x_{N}(t)\right)$. By letting $t$ run from $0 \rightarrow \infty, \mathbf{x}(t)$ will trace out a trajectory in the $N$-dimensional state space. To determine whether a system is chaotic or not, we consider the time evolution of a small, arbitrary perturbation $\delta \mathbf{x}(0)$ added to the initial condition $\mathbf{x}(0)$. A chaotic trajectory, $\mathbf{x}(t)$, is then characterized by an initially exponential increase of this perturbation, i.e.

$$
\left|\frac{\delta \mathbf{x}\left(t_{2}\right)}{\delta \mathbf{x}\left(t_{1}\right)}\right| \approx \exp \left(\lambda\left(t_{2}-t_{1}\right)\right)
$$

for $t_{2}>t_{1}$ and a positive value of $\lambda$. The constant $\lambda$ is called the (largest) Lyapunov exponent, and it being positive is thus one of the defining properties of a chaotic trajectory. Note that we wrote initial exponential divergence, which was to indicate that we cannot say what happens to the perturbation when it gets big, only that it will increase rapidly when it is small. Another condition for a chaotic trajectory is that it should be bounded [4], which most of physical relevance are. To understand this condition, one can easily imagine two trajectories which diverge exponentially from each other as they move towards infinity, but still

\footnotetext{
${ }^{10}$ Lorenz initially used a seagull instead of a butterfly in the analogy, which I think most people would agree does not have the same poetic quality.
} 
behave in a regular and predictable way. The third and last condition is that the trajectory cannot be a fixed point, periodic, or quasi-periodic, nor approach any of these asymptotically. The two former trajectories are probably well known to the reader, but quasi-periodic trajectories may not be, which we now will discuss in the context of integrable Hamiltonian models.

\subsubsection{Integrable Models and the KAM Theorem}

An integrable Hamiltonian system [13,59] has as many conserved quantities (or integrals of motion) as degrees of freedom, with some additional conditions that should be fulfilled. The conserved quantities are e.g. not allowed to be linear combinations of each other and they must also be in involusion, meaning that their mutual Poisson brackets are zero $[13,58]$. The integrability of the continuous models discussed in connection with solitons in Sec. 1.2.2 is thus a generalization to systems with an infinite number of degrees of freedom. If the system possesses these conserved quantities, then it is possible to make a canonical transformation to action-angle variables, $P_{j}$ and $Q_{j}$, which have the property that

$$
\begin{array}{r}
Q_{j}=\omega_{j} t+A_{j} \\
P_{j}=B_{j},
\end{array}
$$

where $\omega_{j}, A_{j}$ and $B_{j}$ are constants [59]. $B_{j}$ are associated with the conserved quantities, and $Q_{j}$ will, as the name implies, behave like an angle, so that one can add a multiple of $2 \pi$ without changing the system. This describes either a periodic trajectory, if all $\omega_{j} / \omega_{k}$ are rational numbers, and otherwise quasi-periodic, which actually is the case most often since the irrational numbers are much more 'common' than the rational. A quasi-periodic trajectory will thus never return to its initial condition, but it will come arbitrarily close to it. Periodic and quasiperiodic trajectories lie on surfaces in phase space that are topologically equivalent to tori (donut-shapes), where a quasi-periodic trajectory fills up the torus densely. Neither are therefore classified as chaotic, since they are predictable in the sense that they always will be found on their torus. Since an integrable system only can have periodic or quasi-periodic trajectories, it cannot be chaotic.

Are periodic and quasi-periodic trajectories then restricted to integrable systems? This question is usually addressed with the Kolmogorov-Arnold-Moser (KAM) theorem, which considers perturbations of integrable models. Let $H_{0}$ be an integrable Hamiltonian, to which we add $\epsilon H_{1}$, which makes the new Hamiltonian $H=H_{0}+\epsilon H_{1}$ non-integrable (for $\epsilon \neq 0$ ). The parameter $\epsilon$ in a sense indicates the 'degree' of non-integrability. We will not give a mathematically rigorous definition of the KAM theorem, but it essentially states that for sufficiently small $\epsilon$ most tori survive [13], and as $\epsilon$ is turned up from zero, these KAM tori are gradually destroyed. Periodic and quasi-periodic trajectories are thus not restricted to integrable systems, and as it turns out, integrable systems are exceptional and rare, whereas especially quasi-periodic motion is generic. An implication of the KAM theorem is that the phase space sometimes can be divided into regions that are chaotic, and regions that are filled with KAM tori. This is called soft chaos, 
in contrast to hard chaos which means that the whole phase space is filled with chaotic trajectories.

\subsubsection{Stability}

\section{Fixed Points}

Fixed points are often important in their own right, but they can also play an important role when determining the global structure of the phase space. Finding the fixed points, and analyzing the trajectories in their vicinity, is therefore often an important part of studying the dynamics of a system. Once a fixed point has been found, the local behavior can be determined by a linear stability analysis. Let us assume that we have a system where the dynamics is determined by

$$
\begin{gathered}
\dot{x}_{1}=f_{1}\left(x_{1}, x_{2}, \ldots, x_{N}\right) \\
\dot{x}_{2}=f_{2}\left(x_{1}, x_{2}, \ldots, x_{N}\right) \\
\vdots \\
\dot{x}_{N}=f_{N}\left(x_{1}, x_{2}, \ldots, x_{N}\right),
\end{gathered}
$$

or, with a more compact notation,

$$
\dot{\mathbf{x}}=\mathbf{f}(\mathbf{x}) \text {. }
$$

The Hamiltonian formulation of mechanics gives this format ('system form') of the equations directly, where $N / 2$ of the variables are conjugated coordinates and the other $N / 2$ conjugated momenta. The variables $x_{j}$ are real, so if the system has complex degrees of freedom, then they have to be split up into their real and imaginary part. Consider a fixed point $\tilde{\mathbf{x}}$ of the system, for which $\dot{\tilde{\mathbf{x}}}=\mathbf{f}(\tilde{\mathbf{x}})=0$. To determine whether this is an unstable or stable fixed point, we look at the time-evolution of a small perturbation, $\delta \mathbf{x}$, added to $\tilde{\mathbf{x}}$. This can be determined from $\dot{\tilde{\mathbf{x}}}+\dot{\delta} \mathbf{x}=\mathbf{f}(\tilde{\mathbf{x}}+\delta \mathbf{x})$, which, since the perturbation is assumed to be small, can be Taylor expanded, leading to (since $\dot{\tilde{\mathbf{x}}}=\mathbf{f}(\tilde{\mathbf{x}})=0$ )

$$
\dot{\delta} \mathbf{x}=\mathbf{D f}(\tilde{\mathbf{x}}) \delta \mathbf{x}
$$

where $\mathbf{D f}(\tilde{\mathbf{x}})$ is the Jacobian or functional matrix

$$
\operatorname{Df}(\tilde{\mathbf{x}})=\left(\begin{array}{cccc}
\partial f_{1} / \partial x_{1} & \partial f_{1} / \partial x_{2} & \cdots & \partial f_{1} / \partial x_{N} \\
\partial f_{2} / \partial x_{1} & \partial f_{2} / \partial x_{2} & \cdots & \partial f_{2} / \partial x_{N} \\
\vdots & \vdots & \ddots & \vdots \\
\partial f_{N} / \partial x_{1} & \partial f_{N} / \partial x_{2} & \cdots & \partial f_{N} / \partial x_{N}
\end{array}\right)
$$

evaluated at the fixed point $\tilde{\mathbf{x}}$. Let us denote the eigenvectors and eigenvalues of the functional matrix with $\mathbf{v}_{j}$ and $h_{j}$, respectively, so that

$$
\operatorname{Df}(\tilde{\mathbf{x}}) \mathbf{v}_{j}=h_{j} \mathbf{v}_{j}
$$


Consider now a perturbation which is parallel to an eigenvector, $\mathbf{v}_{j}$. Its time evolution is determined by $\delta \dot{\mathbf{x}}=h_{j} \delta \mathbf{x}$, which has the solution

$$
\delta \mathbf{x}(t)=\delta \mathbf{x}(0) e^{h_{j} t} .
$$

It will thus stay parallel to the eigenvector, but grow if $h_{j}$ is a positive real number or shrink if it is a negative real number. The eigenvalues can also be complex, but these will always appear in complex conjugated pairs, since the functional matrix is real. A perturbation in the plane spanned by the corresponding eigenvectors will then spiral, either towards $\tilde{\mathbf{x}}$ if the real part of the eigenvalues are negative, or away from $\tilde{\mathbf{x}}$ if they are positive. Should the eigenvalues be purely imaginary then the perturbation will circle the fixed point in a periodic orbit. This is called an elliptic fixed point. Assuming that the eigenvectors span the whole phase space, a general perturbation can be written as $\delta \mathbf{x}(0)=\sum_{j} c_{j} \mathbf{v}_{j}$, which will evolve as

$$
\delta \mathbf{x}(t)=\sum_{j} c_{j} e^{h_{j} t} \mathbf{v}_{j} .
$$

Since a random perturbation almost certainly will have at least a small component in each eigen-direction, it is enough that only one eigenvalue has a positive real part for the fixed point to be unstable.

The reasoning above is valid for both conservative and dissipative systems, but if a system is Hamiltonian, then we also have to account for Liouville's theorem, which states that all phase space volumes are dynamically preserved [59]. This means that if one follows not the time evolution of a single point, but instead of a 'blob' of points in phase space, then the volume of this blob will not change. This will limit the type of fixed points that are allowed for a Hamiltonian system. There can for instance not be a fixed point attractor with only negative eigenvalues, since this would correspond to a volume shrinkage towards the fixed point. More specifically, the functional matrix of a Hamiltonian systems is infinitesimally symplectic $^{11}$, and the eigenvalues of such matrices have a special property, namely that if $h_{j}$ is an eigenvalue, then so are also $-h_{j}, h_{j}^{*}$, and $-h_{j}^{*}[60]$. This guarantees that all eigenvalues appear in pairs which sum to zero. A Hamiltonian system is therefore linearly unstable unless all eigenvalues of the functional matrix reside on the imaginary axis, since if there is an eigenvalue that has a negative real part, then there must also be one with a positive real part ${ }^{12}$.

The classical pendulum can serve as an illustrative example. It has an unstable fixed point when it points straight up, since it is possible in principle to balance the pendulum like this, but even the slightest perturbation will cause the pendulum to swing around and thus deviate strongly from the fixed point. It is however possible, at least in theory, to displace the pendulum slightly and give it a little push so that it ends pointing straight up (it will take an infinite amount of time to reach this position), but this is of course extremely unlikely. This would correspond to a

\footnotetext{
${ }^{11}$ A matrix $M$ is infinitesimally symplectic if $M^{T} J+J M=0$, where $J$ is a skew-symmetric matrix $J^{T}=-J$ ( $T$ denotes matrix transpose), and $M^{T}$ is thus similar to $-M$.

${ }^{12}$ Sometimes the imaginary number $i$ is extracted from the stability eigenvalues $h_{j}$, meaning that linear instability is associated with their imaginary part.
} 
perturbation entirely in the direction of an eigenvector with a negative eigenvalue. The pendulum pointing straight down corresponds instead to a stable fixed point, where it still will remain in the vicinity of the fixed point if it is being perturbed, i.e. given a small push.

Consider now a Hamiltonian system with a stable fixed point. If the system's parameters are being changed, then this fixed point can become unstable in essentially two ways. Either a pair of eigenvalues collides at the origin and goes out along the real axis. The other option is that two pairs of eigenvalues collide, one pair colliding at a positive imaginary value and the other at the corresponding negative imaginary value, and go out in the complex plane. The latter type is called a Hamiltonian Hopf bifurcation (see e.g. [61] and references therein) and leads to an oscillatory instability, meaning that a perturbation from the fixed point will oscillate around it with an exponentially increasing amplitude. A bifurcation means that the behavior of the system changes, which in the case of the Hamiltonian Hopf bifurcation is that a stable fixed point becomes unstable. Ordinary Hopf bifurcations (or supercritical Hopf bifurcations) occur in dissipative systems where the real part of a complex eigenvalue pair goes from negative to positive, turning a stable oscillatory fixed point into a stable periodic orbit surrounding the fixed point [13]. In paper II we studied quantum signatures of oscillatory instabilities and Hamiltonian Hopf bifurcations of the DNLS trimer, originally studied in Ref. [61] (see also Secs. 3.6 and 4.7).

\section{Periodic Trajectories - Floquet Analysis}

The linear stability analysis of a periodic trajectory is often called Floquet analysis. Let us assume that $\mathbf{x}(t)$ is periodic so that $\mathbf{x}(t+T)=\mathbf{x}(t)$, and that its evolution is given by Eq. (1.29). We again consider how a small perturbation $\delta \mathbf{x}(0)$ evolves, which is determined from

$$
\delta \dot{\mathbf{x}}(t)=\mathbf{f}(\mathbf{x}(t)+\delta \mathbf{x}(t))-\mathbf{f}(\mathbf{x}(t))
$$

where $\mathbf{f}(\mathbf{x}+\delta \mathbf{x})$ is linearized in $\delta \mathbf{x}$. By integrating Eq. (1.36) from $t=0$ to $t=T$ for $N$ linearly independent perturbations, we can obtain

$$
\delta \mathbf{x}(T)=\mathcal{F}_{T} \delta \mathbf{x}(0)
$$

which gives $\delta \mathbf{x}(T)$ for an arbitrary initial perturbation $\delta \mathbf{x}(0) . \quad \mathcal{F}_{T}$ is an $N \times N$ matrix called the Floquet matrix, which in Floquet analysis plays a role that is similar to that of the functional matrix in the linear stability analysis of a fixed point. Note that when we integrate Eq. (1.36) to obtain $\mathcal{F}_{T}$, we also need to have $\mathbf{x}(t)$ for $t=0 \rightarrow T$, which effectively acts as a periodic potential for the perturbation.

Assuming that $\mathbf{u}_{j}(0)$ is an eigenvector to $\mathcal{F}_{T}$ with eigenvalue $g_{j}$, from Eq. (1.37) we can deduce that $\mathbf{u}_{j}(m T)=g_{j}^{m} \mathbf{u}_{j}(0), m \in \mathbb{Z}^{+}$(note that this utilizes the linearity of Eq. (1.36)). A perturbation in this eigen-direction is thus growing with time if $\left|g_{j}\right|>1$, meaning that the periodic orbit is linearly unstable if any eigenvalue lies outside of the unit circle in the complex plane. The eigenvalues 
$g_{j}$ are called Floquet multipliers. For periodic orbits there is always a Floquet multiplier with algebraic multiplicity two and geometric multiplicity one that is equal to unity, corresponding to a perturbation in the direction of the trajectory [62]. For Hamiltonian systems, the Floquet matrix is a symplectic matrix, which has the property that if $g_{j}$ is an eigenvalue, then so are also $g_{j}^{*}, 1 / g_{j}$, and $1 / g_{j}^{* 13}$ [60]. A periodic orbit for a Hamiltonian system is thus linearly unstable unless all Floquet multipliers lie on the unit circle. These concepts are applied to the DNLS model in Sec. 3.1.1.

\subsection{Classical vs Quantum Mechanics}

One of the main themes of this thesis is the connection between classical and quantum mechanical models. Quantum mechanics is typically said to be the physics of microscopic objects such as atoms and molecules, while classical mechanics instead describes the macroscopic items that we encounter in our everyday life. But even macroscopic objects, such as planet earth or a cat, is built up by atoms and molecules, and there must therefore be some connection between quantum and classical mechanics. To address this issue, the great Niels Bohr formulated his correspondence principle which states, a bit vaguely expressed, that in appropriate limits of quantum mechanics, classical physics must arise. It can also be expressed that for a quantum system with a classical analog, expectation values of operators will behave like the corresponding classical quantity in the limit $\hbar \rightarrow 0$ [63].

Quantum mechanics is a theory that is based around linear eigenvalue equations, such as the Schrödinger equation, and a relevant question is how to quantum mechanically describe classical concepts that are fundamentally connected to the nonlinearity of the classical equation, which we have seen some examples of in this chapter. As we will see an example of in chapter 4 , it is the interactions between particles that result in nonlinearities in the classical model.

An example of the fundamental difference between classical and quantum mechanics is for periodic potentials, where for the linear quantum theory we have the Bloch theorem [5]. According to this theorem, the eigenstates must be functions with the same periodicity as the lattice modulated by a plane wave, implying that expectation values cannot depend on which site one looks at, which seems to be in contradiction with the existence of DBs. How to reconcile this is addressed in Sec. 4.6.

The field of Quantum Chaos [58] deals with signatures that can be seen in a quantum model which has a classical analog that is chaotic. We will not give any details on this vast field, but just mention a few of the conceptual differences between classical and quantum mechanics that are especially relevant. A central concept in classical chaos is the SIC and that nearby trajectories diverge from each other. In quantum mechanics we however have the Heisenberg uncertainty principle, which puts a fundamental limit on the resolution of the phase space.

Another related field is that of Quantum Optics [64], which as the name implies,

\footnotetext{
${ }^{13}$ The defining property of a symplectic matrix $M$ is that $M^{T} J M=J$, where $J$ again is skewsymmetric. $M^{T}$ is therefore similar to $M^{-1}$ which gives the relation between the eigenvalues.
} 
is connected to how to quantum mechanically describe different states of light, both with and without classical analogs, using photons. Some of the concepts that we will use, e.g. coherent states (see Sec. 4.4), have played very prominent roles in quantum optics. The two different descriptions of light - quantum mechanically in terms of photons and classically in the form of a field - are analogous to how the quantum Bose-Hubbard model of chapter 4 and the classical DNLS equation of chapter 3 describe Bose-Einstein condensates in optical lattices. An important difference is that photons are massless particles that can be created and destroyed, so that their number are not conserved. 


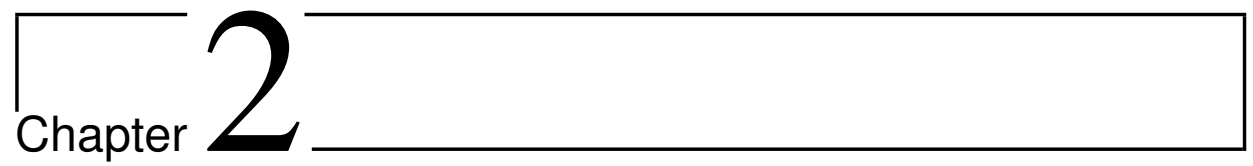

\section{Physical Systems}

\subsection{Bose-Einstein Condensates}

It is a remarkable property of nature that all particles can be classified as either fermions or bosons. Fermions are particles which have half integer spin and obey the Pauli exclusion principle, meaning that each quantum state can be occupied with at most one fermion, while bosons on the other hand have integer spin, and any number of bosons can populate a given quantum state. In this thesis, we will deal primarily with the latter type.

The statistical properties of bosons were worked out by the Indian physicist Satyendra Nath Bose (whom they are named after) and Albert Einstein in 19241925 [65-67]. It was Einstein who realized that a macroscopic fraction of noninteracting massive bosons will accumulate in the lowest single-particle quantum state at sufficiently low temperatures. This new phase of matter is what we today call a 'Bose-Einstein condensate' (BEC). The condensed atoms can be described by a single wave function, thus making the intriguing, and normally microscopic, wave-like behavior of matter in quantum mechanics a macroscopic phenomenon.

BECs were for a long time considered to be merely a curiosity with no practical importance, until in 1938 when Fritz London [68] suggested that the recently discovered superfluidity of liquid ${ }^{4} \mathrm{He}$ could be explained by using this concept. Also the theory of superconductivity builds on the notion of a BEC, this time of electron pairs (Cooper pairs). These are however two strongly interacting systems, and the concept of a BEC becomes quite more complicated than the simple scenario of noninteracting particles originally considered by Einstein ${ }^{1}$. Also, only about $10 \%$ of the atoms in liquid ${ }^{4} \mathrm{He}$ are in the condensed phase. But to realize a purer $\mathrm{BEC}$ closer to the original idea would prove to be a formidable task, due to the extremely low temperatures it requires.

In the 1970's, new powerful techniques, using magnetic fields and lasers, were developed to cool neutral atoms. This lead to the idea that it would be possible to

\footnotetext{
${ }^{1}$ For a more formal definition of a BEC, see [69].
} 
realize BECs with atomic vapors. But, as everyone knows, if a normal gas is cooled sufficiently, it will eventually form a liquid or solid, two states where interactions are of great importance. This can however be overcome by working with very dilute gases, so that the atoms stay in the gaseous form when they are cooled down, but the diluteness also implies the need for temperatures in the micro- or nanokelvin scale for condensation ${ }^{2}$.

Spin-polarized hydrogen was proposed as an early candidate ${ }^{3}$ [71], but with the development of laser cooling, which cannot be used on hydrogen, alkali atoms also entered the race. Finally in 1995, some seventy years after Einstein's original proposal, a BEC was observed in a gas of rubidium atoms, cooled to a temperature of 170 nanokelvin [72]. This was only a month prior to another group observing it in lithium [73], and later the same year it was also produced with sodium [74]. This would eventually render Carl Wieman, Eric Cornell (Rb-group) and Wolfgang Ketterle (Na-group) the 2001 Nobel Prize in physics. BECs have since been produced with a number of different atom species, notably with hydrogen in 1998 [75], and are nowadays produced routinely in many labs around the world.

\subsubsection{Theoretical Treatment}

Even for a dilute gas of neutral atoms there are some interactions. The theoretical treatment of these can however be significantly simplified at low temperatures, since they then can be taken to be entirely due to low-energy binary collisions, completely characterized by only a single parameter - the $s$-wave scattering length $a_{s}$. This scattering length is first of all positive for certain elements (e.g. ${ }^{23} \mathrm{Na}$ and ${ }^{87} \mathrm{Rb}$ ) and negative for others (e.g. ${ }^{7} \mathrm{Li}$ ), meaning repulsive and attractive interactions respectively, but it can also, by means of Feshbach resonances, be controlled externally with magnetic field [76]. The many-body Hamiltonian is under these assumptions given by

$$
\hat{H}=\sum_{j}\left[\frac{\mathbf{p}_{j}^{2}}{2 m}+V\left(\mathbf{r}_{j}\right)+\sum_{i<j} U_{0} \delta\left(\mathbf{r}_{i}-\mathbf{r}_{j}\right)\right] .
$$

Here, $U_{0}=4 \pi \hbar^{2} a_{s} / m$ is the strength of the inter-particle contact interaction, while $V(\mathbf{r})$ contains all the externally applied potentials, which often includes a contribution from a harmonic trapping potential with frequency $\omega_{T} \sim 10-1000$ $\mathrm{Hz}$ (cf. $\left.V(x)=m \omega^{2} x^{2} / 2\right)$. Other types of external potentials will be treated in the next section. With the original BEC concept in mind, a Hartree approach is adopted, where the trial many-body wave function $\tilde{\Phi}\left(\mathbf{r}_{1}, \ldots, \mathbf{r}_{N}\right)$ is written as a product of the same normalized single-particle function $\Phi(\mathbf{r})$ (to be determined),

$$
\tilde{\Phi}\left(\mathbf{r}_{1}, \ldots, \mathbf{r}_{N}\right)=\prod_{j=1}^{N} \Phi\left(\mathbf{r}_{j}\right) .
$$

\footnotetext{
${ }^{2}$ The critical temperature of condensation can be estimated by when the thermal de Broglie wavelength $\left(\sim T^{-1 / 2}\right)$ becomes equal to inter-particle spacing [9].

${ }^{3}$ Hydrogen was proposed as a candidate already in 1959 by Hecht [70], but this work was well before its time and went largely unnoticed by the scientific community.
} 
Note that this wave function is symmetric under particle exchange, and thus obeys the Pauli principle for bosons. The average energy of this trial function is

$$
E=N \int\left(\frac{\hbar^{2}}{2 m}|\nabla \Phi(\mathbf{r})|^{2}+V(\mathbf{r})|\phi(\mathbf{r})|^{2}+\frac{N-1}{2} U_{0}|\Phi(\mathbf{r})|^{4}\right) d \mathbf{r},
$$

where the term $N(N-1) / 2$ accounts for the number of boson pairs. By introducing $\Psi(\mathbf{r})=\sqrt{N} \Phi(\mathbf{r})$, which can be interpreted as the wave function of the condensed state (the density of particles given by $n(r)=|\Psi(\mathbf{r})|^{2}$ ), the energy takes the form

$$
E=\int\left(\frac{\hbar^{2}}{2 m}|\nabla \Psi(\mathbf{r})|^{2}+V(\mathbf{r})|\Psi(\mathbf{r})|^{2}+\frac{1}{2} U_{0}|\Psi(\mathbf{r})|^{4}\right) d \mathbf{r}
$$

where the term of order $N^{-1}$ has been neglected. The 'optimal' wave function $\Psi(\mathbf{r})$ for the condensate is found through the standard procedure of minimizing this energy, now with respect to $\Psi^{*}(\mathbf{r})$. We must also account for the constraint that the total number of particles is equal to $N$ :

$$
\int|\Psi(\mathbf{r})|^{2} d \mathbf{r}=N
$$

which is done by introducing a Lagrange multiplier $\mu$ (the chemical potential). The resulting equation reads

$$
\left(-\frac{\hbar^{2} \nabla^{2}}{2 m}+V(\mathbf{r})+U_{0}|\Psi(\mathbf{r})|^{2}\right) \Psi(\mathbf{r})=\mu \Psi(\mathbf{r}),
$$

which is called the time-independent Gross-Pitaevskii equation. As discussed in Sec. 1.2.2 this is an NLS type equation. The validity of this description relies on extremely low temperatures, and that the scattering length $a_{s}$ is much smaller than the average inter-particle spacing. Similar to the ordinary Schrödinger equation, there is also a time-dependent Gross-Pitaevskii equation

$$
i \hbar \frac{\partial \Psi(\mathbf{r}, t)}{\partial t}=\left(-\frac{\hbar^{2} \nabla^{2}}{2 m}+V(\mathbf{r}, t)+U_{0}|\Psi(\mathbf{r}, t)|^{2}\right) \Psi(\mathbf{r}, t) .
$$

The individual behavior of the condensed atoms is 'smeared out' in $\Psi$, and the validity of the Gross-Pitaevskii equation therefore relies on the number of particles in the condensate being so large that quantum fluctuations can be neglected. The Gross-Pitaevskii equation has been very successful in describing many macroscopic properties [9], especially for BECs in traps, which was the focus of much of the early research after the first experimental realization [77]. Both dark [78,79] and bright solitons [80,81], as well as vortices [82], have been experimentally observed in BECs.

But just like with electromagnetism, where certain phenomena can be explained with classical electromagnetic fields and others need a more detailed, quantum mechanical, description with photons, it is sometimes necessary to use a more microscopic (i.e. quantum mechanical) treatment also for BECs (cf. Sec. 4.2). One example, as mentioned earlier, is when there are few particles in the condensate. 


\subsubsection{Bose-Einstein Condensates in Optical Lattices}

Utilizing optical lattices - periodic structures generated by the interference of laser beams - has made it possible to study effects of periodic potentials on BECs. The physical mechanism behind this can be illustrated by considering the simple case of two counter-propagating beams with the same amplitude, linear polarization and frequency $\omega$. The beams will together form a standing wave with electric field ${ }^{4}$ $E(x, t) \propto \cos (\omega t) \cos (k x)$, which will induce electric dipole moments in atoms placed in the field. This dipole moment will in its turn couple back to the electric field, leading to a spatially varying (AC-Stark) shift of the atomic energy level, equal to

$$
\Delta E(x)=-\frac{1}{2} \alpha(\omega)\left\langle E^{2}(x, t)\right\rangle,
$$

where $\alpha$ denotes the atomic polarizability and $\langle$.$\rangle the average over one period of$ the laser [83]. All in all, this leads to a one-dimensional periodic potential for the atoms, with minima at either the nodes or antinodes of the standing wave, depending on the polarizability of the atoms. Adding more lasers makes it possible to create lattices, not only simple types with higher dimensionality, but also of a more complex character, e.g. moving lattices, kagomé lattices and quasi-periodic lattices $[83,84]$. It is also possible to strongly suppress the atoms' movement in a given direction, by increasing either the trapping or periodic potential that is pointing in that way. By doing this, (quasi-)one or two-dimensional systems can be constructed, where the atoms are confined to move along either one-dimensional tubes or two-dimensional disks.

One of the most appealing features of experiments with BECs in optical lattices is the great external controllability of many of the system's parameters, making it possible to access fundamentally different physical regimes. One example of this was mentioned above with the different lattice geometries that are possible to create. Another is the potential depth, which is tuned by adjusting the laser intensity and can be varied over a wide range - from completely vanished lattices to very deep ones with sites practically isolated from each other. The potential depth can on top of it all also be changed in real time, making it possible to study phase transitions in detail [85] (cf. Sec. 4.9).

Deeper potentials will also lead to a larger confinement (and therefore higher density) within the lattice wells, resulting in stronger on-site interactions, and it is actually possible to reach the strongly interacting regime by increasing the depth. The interaction strength, and sign, can also be changed with Feshbach resonances.

Finally, the number of particles per lattice site in experiments can vary from so few that quantum fluctuations are important [76] to sufficiently many for meanfield theories to be applicable [83]. In the former case, it is necessary to use fully quantum mechanical models, for instance the Bose-Hubbard which chapter 4 will deal with in detail, while one generally uses models based on the Gross-Pitaevskii equation (2.7) in the latter case (see also Sec. 3.2).

The diversity, and high accuracy, of experiments with BECs in optical lattices, makes it an ideal test-bed for many theoretical predictions, for instance from

\footnotetext{
${ }^{4}$ Effects of the magnetic field can generally be neglected.
} 
condensed matter physics (so called quantum simulators [86]).

Nonlinear localized BECs have been experimentally observed in both weak [87] and deep [88] one-dimensional optical lattices, both using repulsive atoms. In the former case, gap solitons were generated with the help of the BEC's anomalous dispersion ${ }^{5}$, which is produced by the periodic potential [87]. In the second case, the BEC gets a localized core with steep, high amplitude boundaries, through which the tunneling of atoms is suppressed [88].

\subsection{Optical Waveguide Arrays}

Optical waveguides are, as the name implies, structures designed to guide electromagnetic waves along them. There are several different types of waveguides [89], but our focus is on dielectric waveguides, where a core of some dielectric material, which the electromagnetic wave mainly is confined to, is surrounded by another material with a lower refractive index, called the cladding ${ }^{6}$. An analogy from everyday life would be a hose that guides the flow of water, with the important difference that there is an evanescent electromagnetic field outside the dielectric waveguide's core that decays (typically exponentially) with the distance from it.

To describe waveguides we start with Maxwell's equations [89], which in a source-free environment have the form (in SI-units)

$$
\begin{array}{r}
\boldsymbol{\nabla} \cdot \mathbf{D}=0, \\
\nabla \cdot \mathbf{B}=0, \\
\nabla \times \mathbf{E}=-\frac{\partial \mathbf{B}}{\partial t}, \\
\nabla \times \mathbf{H}=\frac{\partial \mathbf{D}}{\partial t} .
\end{array}
$$

$\mathbf{D}$ is the displacement field, which is related to the electric field intensity by $\mathbf{D}=$ $\epsilon \mathbf{E}=\epsilon_{0} \mathbf{E}+\mathbf{P}$, where $\epsilon$ is the dielectric constant and $\mathbf{P}$ the polarization field. $\epsilon$ and $\mathbf{P}$ indicate how the dielectric responds to an applied electric field through induced dipole moments in its molecules. $\mathbf{H}=\mathbf{B} / \mu$ is the magnetizing field, and $\mu$ the magnetic permeability. The refractive index $n$ of the dielectric is related to the material parameters $\epsilon$ and $\mu$ by $n=\sqrt{\mu_{0} \epsilon_{0} / \mu \epsilon}$, so that $v=c / n$ is the velocity of an electromagnetic wave in (an infinite sample) of the medium, where $c$ as usual is the speed of light in vacuum. We will take the dielectric's magnetic permeability to be the same as in vacuum, $\mu=\mu_{0}$.

By taking the curl of Eq. (2.9c), and combining it with Eq. (2.9d), one can derive the following wave equation

$$
\nabla^{2} \mathbf{E}-\mu \epsilon_{0} \frac{\partial^{2} \mathbf{E}}{\partial t^{2}}=\mu \frac{\partial^{2} \mathbf{P}}{\partial t^{2}}
$$

\footnotetext{
${ }^{5}$ Anomalous dispersion means that $\partial^{2} E / \partial q^{2}<0$, where $E$ and $q$ are energy and wavenumber, respectively.

${ }^{6}$ There are mainly two types of dielectric waveguides. Step-index where the refractive index in the core is constant, and graded-index where the refractive index increases gradually.
} 
We will later use a perturbation theory called coupled mode theory [90] (see Sec. 2.2.1) to treat the nonlinear properties of the dielectric. Let us therefore first consider the linear case when $\epsilon$ is a constant, and (2.10) can be reduced to

$$
\nabla^{2} \mathbf{E}-\mu \epsilon \frac{\partial^{2} \mathbf{E}}{\partial t^{2}}=0
$$

Assuming that we have an optical waveguide pointing in the $z$-direction with a uniform cross section, we make the ansatz

$$
\mathbf{E}(x, y, z, t)=\mathcal{E}(x, y) e^{\gamma z-i \omega t}
$$

which inserted in Eq. (2.11) gives

$$
\nabla_{x y}^{2} \mathcal{E}(x, y)+h^{2} \mathcal{E}(x, y)=0
$$

where

$$
h^{2}=\gamma^{2}+\mu \epsilon \omega^{2},
$$

and

$$
\nabla_{x y}^{2}=\frac{\partial^{2}}{\partial x^{2}}+\frac{\partial^{2}}{\partial y^{2}}
$$

is the transverse Laplacian. Equation (2.13) is a vector Helmholtz equation. The propagation constant $\gamma=\alpha+i \beta$ describes an oscillatory and propagating solution if it is imaginary, and a solution that decays in the propagation direction if it is real (a positive $\alpha$ is a nonphysical solution). With the corresponding ansatz for the magnetic field, $\mathbf{H}(x, y, z, t)=\mathcal{H}(x, y) e^{\gamma z-i \omega t}$, the Helmholtz equation can also be derived for $\mathcal{H}$ :

$$
\boldsymbol{\nabla}_{x y}^{2} \mathcal{H}(x, y)+h^{2} \mathcal{H}(x, y)=0 .
$$

By inserting these ansätze for $\mathbf{E}(x, y, z, t)$ and $\mathbf{H}(x, y, z, t)$ also into equations $(2.9 \mathrm{c})$ and $(2.9 \mathrm{~d})$, after some manipulation we can arrive at

$$
\begin{aligned}
\mathcal{H}_{x} & =-\frac{1}{h^{2}}\left(\gamma \frac{\partial \mathcal{H}_{z}}{\partial x}-i \omega \epsilon \frac{\partial \mathcal{E}_{z}}{\partial y}\right) \\
\mathcal{H}_{y} & =-\frac{1}{h^{2}}\left(\gamma \frac{\partial \mathcal{H}_{z}}{\partial y}+i \omega \epsilon \frac{\partial \mathcal{E}_{z}}{\partial x}\right) \\
\mathcal{E}_{x} & =-\frac{1}{h^{2}}\left(\gamma \frac{\partial \mathcal{E}_{z}}{\partial x}+i \omega \mu \frac{\partial \mathcal{H}_{z}}{\partial y}\right) \\
\mathcal{E}_{y} & =-\frac{1}{h^{2}}\left(\gamma \frac{\partial \mathcal{E}_{z}}{\partial y}-i \omega \mu \frac{\partial \mathcal{H}_{z}}{\partial x}\right)
\end{aligned}
$$

where $\mathcal{E}_{j}$ and $\mathcal{H}_{j}(j=x, y, z)$ denote the components of either vector. These equations state that the transverse components of the fields can be determined by the components that point in the propagation direction. $\mathcal{E}_{z}$ and $\mathcal{H}_{z}$ are thus first obtained from equations (2.13) and (2.16), which are then used in Eqs. (2.17) to determine the transverse components. 
$\mathcal{E}_{z}$ and $\mathcal{H}_{z}$ must also satisfy the boundary conditions of the waveguide ${ }^{7}$. This leads to two different types of solutions for $\mathcal{E}_{z}$ and $\mathcal{H}_{z}$. First, there will be a finite number of confined modes (or guiding modes) for a discrete set of $h^{2}$. These are the solutions of primary interest as they represent a confinement of the field to the waveguide. Second, there will be an infinite number of unbounded radiation modes for $h^{2}$ above a certain threshold, which represent a transport of energy away from the waveguide ${ }^{8}$. The different modes, labeled with superscript $\nu, \lambda$, are orthogonal to each other [89],

$$
\iint \mathcal{E}^{(\nu) *} \cdot \mathcal{E}^{(\lambda)} d x d y=\iint \mathcal{H}^{(\nu) *} \cdot \mathcal{H}^{(\lambda)} d x d y=0, \quad \lambda \neq \nu,
$$

where the integral runs over the cross section of the waveguide.

For a given eigenvalue $h^{2}$, the relation $(2.14) h^{2}=\gamma^{2}+\mu \epsilon \omega^{2}$ gives the dependence (dispersion relation) of $\gamma$ on $\omega$. From this we find that the phase velocity of a wave that travels in the waveguide is higher compared to in an unbounded sample, but that the group velocity is lower. There is also a cut-off frequency at $\omega=h / \sqrt{\mu \epsilon}$, below which $\gamma$ becomes real and the mode associated with this $h$ is not propagating but decaying into the waveguide. The frequency can thus be chosen so that only one or a few modes are propagating, called single-mode and multi-mode propagation, respectively.

For waveguides that consist of a dielectric core surrounded by a conductor ${ }^{9}$, it is always possible to find transverse electric (TE) modes and transverse magnetic (TM) modes, for which $\mathcal{E}_{z}=0$ and $\mathcal{H}_{z}=0$, respectively. For dielectric waveguides this is generally not the case, where the modes generally have both a nonzero $\mathcal{E}_{z}$ and $\mathcal{H}_{z}$ (called hybrid modes). There are however some cases when TE and TM modes can be found for dielectric waveguides, for example in slab waveguides and azimuthally symmetric modes in circular waveguides.

For TE and TM modes, their normalization factor can be calculated explicitly, i.e. the values of the integrals in (2.18) for $\nu=\lambda$. It seems natural that these will depend on the input power of the waves, so let us consider the Poynting vector associated with a TE or TM mode $\mathbf{S}^{(\nu)}=\left(\mathbf{E}^{(\nu)} \times \mathbf{H}^{(\nu)}\right) / 2$, which gives the energy flux density for the mode. The total power flow $P^{(\nu)}$ along the waveguide is obtained by integrating the Poynting vector's $z$-component over the waveguide's transverse directions, $x$ and $y$. The Poynting vector's $z$-component is given by $\mathbf{S}^{(\nu)} \cdot \hat{\mathbf{z}}=\left(\mathbf{E}_{t}^{(\nu)} \times \mathbf{H}_{t}^{(\nu)}\right) \cdot \hat{\mathbf{z}} / 2$, where hats denote unit vector, and subscript $t$ the transverse projection onto the $x-y$ plane. By inserting that $\mathcal{E}_{z}^{(\nu)}=0$ and $\mathcal{H}_{z}^{(\nu)}=0$ into Eq. (2.17) for TE and TM modes, respectively, the transverse components of the vectors are related by

$$
\mathbf{H}_{t}^{(\nu)}=\frac{1}{Z_{\nu}} \hat{\mathbf{z}} \times \mathbf{E}_{t}^{(\nu)},
$$

\footnotetext{
${ }^{7}$ The tangential components of $\mathbf{E}$ and $\mathbf{H}$ must be continuous over a material boundary [89].

${ }^{8}$ Compare this with the bounded and extended eigenstates of a quantum particle in a box with finite walls.

${ }^{9}$ This type of waveguide can be compared to a quantum particle confined to a box with infinite walls.
} 
where $Z_{\nu}=\mathcal{E}_{x}^{(\nu)} / \mathcal{H}_{y}^{(\nu)}=-\mathcal{E}_{y}^{(\nu)} / \mathcal{H}_{x}^{(\nu)}$ is the wave impedance, equal to

$$
Z_{\nu}= \begin{cases}\frac{\mu \omega}{\beta_{\nu}} & (\mathrm{TE}) \\ \frac{\beta_{\nu}}{\epsilon \omega} & (\mathrm{TM})\end{cases}
$$

Using that $\left(\mathbf{E}_{t}^{(\nu)} \times \mathbf{H}_{t}^{(\nu)}\right) \cdot \hat{\mathbf{z}} / 2=\left(\mathbf{E}_{t}^{(\nu)} \times\left(\hat{\mathbf{z}} \times \mathbf{E}_{t}^{(\nu)}\right)\right) \cdot \hat{\mathbf{z}} / 2 Z_{\nu}=\left|\mathbf{E}_{t}^{(\nu)}\right|^{2} / 2 Z_{\nu}$, the total power becomes

$$
P=\frac{1}{2 Z_{\nu}} \iint\left|\mathbf{E}_{t}^{(\nu)}\right|^{2} d x d y .
$$

In the case of TE modes $\mathbf{E}_{t}^{(\nu)}=\mathbf{E}^{(\nu)}$, meaning that the above integral can be used to write

$$
\iint\left|\mathbf{E}^{(\nu)}\right|^{2} d x d y=2 Z_{\nu} P=\frac{2 P \mu \omega}{\beta_{\nu}} .
$$

For TM modes one also needs to account for the longitudinal component of the electric field when calculating the normalization factor

$$
\iint\left|\mathbf{E}^{(\nu)}\right|^{2} d x d y=\iint\left|\mathbf{E}_{t}^{(\nu)}\right|^{2} d x d y+\iint\left|E_{z}^{(\nu)}\right|^{2} d x d y .
$$

To calculate the last integral, we note that by using Eqs. $(2.17 \mathrm{c})$ and $(2.17 \mathrm{~d})$ (with $\mathcal{H}_{z}=0$ ), we can write

$$
\mathbf{E}_{t}=-\frac{\gamma}{h^{2}} \boldsymbol{\nabla}_{T} E_{z}=-\frac{\gamma}{h^{2}}\left(\hat{\mathbf{x}} \frac{\partial}{\partial x}+\hat{\mathbf{y}} \frac{\partial}{\partial y}\right) E_{z}
$$

Consider now

$$
\begin{array}{r}
\iint\left|\mathbf{E}_{t}\right|^{2} d x d y=\left(\frac{\gamma}{h^{2}}\right)^{2} \iint \nabla_{T} E_{z}^{*} \cdot \nabla_{T} E_{z} d x d y \\
=\frac{\gamma^{2}}{h^{4}}\left[\oint_{C} E_{z}^{*}\left(\frac{\partial E_{z}}{\partial n}\right) d l-\iint E_{z}^{*} \nabla_{T}^{2} E_{z} d x d y\right],
\end{array}
$$

where the last step uses Green's first identity for two dimensions. The first integral within the square bracket is a line integral along the boundary of a cross-sectional surface of the waveguide, and the derivative within this integral vanishes at a dielectric boundary. We are thus left with only the second integral, where we plug in that $\nabla_{T}^{2} E_{z}=-h^{2} E_{z}$ from Eq. (2.13), which gives that

$$
\iint\left|\mathbf{E}_{t}\right|^{2} d x d y=\frac{\gamma^{2}}{h^{2}} \iint\left|E_{z}\right|^{2} d x d y
$$

Inserting this into Eq. (2.23) yields

$$
\iint\left|\mathbf{E}^{(\nu)}\right|^{2} d x d y=\left(1+\frac{h^{2}}{\gamma^{2}}\right) \iint\left|\mathbf{E}_{t}^{(\nu)}\right|^{2} d x d y=\frac{2 \beta_{\nu}^{2}-\mu \epsilon \omega^{2}}{\beta_{\nu}} \frac{2 P}{\epsilon \omega} .
$$




\subsubsection{Coupled Mode Theory}

Coupled mode theory is a perturbation theory developed for optical waveguide systems. The basic idea is to start with a single waveguide with linear refractive index, and consider the influence of e.g. nonlinearity and neighboring waveguides as perturbations on the polarization field. This treatment will effectively couple the guiding modes of different waveguides. Consider again Eq. (2.10) and assume that the 'unperturbed' linear waveguide is described by a dielectric constant $\epsilon$ which relates the electric field to the unperturbed polarization field by $\mathbf{P}_{0}=$ $\left(\epsilon-\epsilon_{0}\right) \mathbf{E}$. Assume further that the system's dielectric constant (and thus the refractive index) is perturbed somehow, e.g. by introducing nonlinearities and/or neighboring waveguides. The electric field $\mathbf{E}$ then becomes associated with a new polarization $\mathbf{P}$, which we express in terms of the old unperturbed polarization $\mathbf{P}_{0}$ and a perturbation polarization $\mathbf{P}_{\text {pert }}$,

$$
\mathbf{P}=\mathbf{P}_{0}+\mathbf{P}_{\text {pert }} .
$$

Inserting this expression into Eq. (2.10), and moving the term containing $\mathbf{P}_{0}$ to the left-hand side, we obtain

$$
\nabla^{2} \mathbf{E}-\mu \epsilon \frac{\partial^{2} \mathbf{E}}{\partial t^{2}}=\mu \frac{\partial^{2} \mathbf{P}_{p e r t}}{\partial t^{2}}
$$

The electric field is now expanded in terms of the confined modes of the unperturbed system

$$
\mathbf{E}(\mathbf{r}, t)=\frac{1}{2} \sum_{\nu} \psi_{\nu}(z) \mathcal{E}^{(\nu)}(x, y) e^{i\left(\beta_{\nu} z-\omega t\right)}+c . c .,
$$

where c.c. denotes complex conjugation. This expression neglects any occupation of radiation modes, so that $\gamma=i \beta_{\nu}$. By substituting (2.29) into Eq. (2.28), we obtain

$$
\begin{array}{r}
\frac{1}{2} \sum_{\nu}\left\{\psi_{\nu}\left[\nabla_{x y}^{2} \mathcal{E}^{(\nu)}+\left(\mu \epsilon \omega^{2}-\beta_{\nu}^{2}\right) \mathcal{E}^{(\nu)}\right]\right. \\
\left.+\left(\frac{\partial^{2} \psi_{\nu}}{\partial z^{2}}+2 i \beta_{\nu} \frac{\partial \psi_{\nu}}{\partial z}\right) \mathcal{E}^{(\nu)}\right\} e^{i\left(\beta_{\nu} z-\omega t\right)}+c . c . \\
=\mu \frac{\partial^{2} \mathbf{P}_{\text {pert }}}{\partial t^{2}} .
\end{array}
$$

The expression inside the square bracket vanishes, as it is the left-hand side of Eq. (2.13). We further assume that $\psi_{\nu}(z)$ is slowly varying, in the sense that

$$
\left|\frac{\partial^{2} \psi_{\nu}}{\partial z^{2}}\right| \ll\left|\beta_{\nu} \frac{\partial \psi_{\nu}}{\partial z}\right|,
$$

simplifying Eq. (2.30) to

$$
\sum_{\nu} i \beta_{\nu} \frac{\partial \psi_{\nu}}{\partial z} \mathcal{E}^{(\nu)} e^{i\left(\beta_{\nu} z-\omega t\right)}+\text { c.c. }=\mu \frac{\partial^{2} \mathbf{P}_{p e r t}}{\partial t^{2}} .
$$


We assume that $\mathcal{E}^{(\nu)}$ are real valued, as they are in many cases [89]. Multiplying Eq. (2.32) by $\mathcal{E}^{(\nu)}$ and integrating over $x$ and $y$, and utilizing the orthogonality of different modes Eq. (2.18), gives

$$
i \frac{\partial \psi_{\nu}}{\partial z} e^{i\left(\beta_{\nu} z-\omega t\right)} \iint\left|\mathcal{E}^{(\nu)}\right|^{2} d x d y+\text { c.c. }=\frac{\mu}{\beta_{\nu}} \frac{\partial^{2}}{\partial t^{2}} \iint \mathcal{E}^{(\nu)} \cdot \mathbf{P}_{\text {pert }} d x d y .
$$

For TE and TM modes, the integral on the left-hand side can be evaluated explicitly with the normalization factors Eqs. (2.22) and (2.26), giving

$$
i \frac{\partial \psi_{\nu}}{\partial z} e^{i\left(\beta_{\nu} z-\omega t\right)}+c . c .=\left\{\begin{array}{l}
\frac{1}{2 \omega P} \frac{\partial^{2}}{\partial t^{2}} \iint \mathcal{E}^{(\nu)} \cdot \mathbf{P}_{\text {pert }} d x d y \\
\frac{1}{2 \omega P} \frac{\mu \epsilon \omega^{2}}{2 \beta_{\nu}^{2}-\mu \epsilon \omega^{2}} \frac{\partial^{2}}{\partial t^{2}} \iint \mathcal{E}^{(\nu)} \cdot \mathbf{P}_{\text {pert }} d x d y
\end{array}\right.
$$

These equations will be used to derive models for waveguide arrays in Sec. 3.3.

\subsubsection{Optical Kerr Effect}

The type of nonlinear dielectric materials that are of interest in this thesis experience what is called the optical Kerr effect (or AC Kerr effect). For an applied electric field

$$
\mathbf{E}=\frac{1}{2}\left(\mathbf{E}(\omega) e^{-i \omega t}+\mathbf{E}(\omega)^{*} e^{i \omega t}\right)
$$

the material's refractive index $n$ will change as

$$
n=n_{0}+n_{2}|E(\omega)|^{2},
$$

where $n_{0}$ is the ordinary low-intensity refractive index, and $n_{2}$ the Kerr index. The 'original' electro-optical Kerr effect, which was discovered by the Scottish physicist John Kerr in the 1870's [91], describes the same dependence for the refractive index as in (2.36) but on static electric fields. Kerr discovered that with an applied static electric field, a dielectric sample can be made birefringent, meaning that when also shining light on it, the experienced refractive index will depend on the directions of polarization and propagation. One result of this is that light that is initially linearly polarized can emerge from the sample elliptically polarized. To emphasize the crucial difference between the two Kerr effects, for the optical Kerr effect it is the light itself that induces the change of refractive index, while for the electrooptical it is achieved with an additional external static field.

To see how the optical Kerr effect can arise, let us consider an expansion of the polarization $\mathbf{P}$ in powers of the light wave's electric field $\mathbf{E}$ :

$$
\mathbf{P}=\epsilon_{0} \chi^{(1)} \mathbf{E}+\epsilon_{0} \chi^{(2)} \mathbf{E} \mathbf{E}+\epsilon_{0} \chi^{(3)} \mathbf{E E E}+\ldots
$$

A few comments about this expansion are necessary. It is first of all not always convergent, for instance if the light is in resonance with some atomic transition, or if the applied field is so strong that photoionization effects come into play (see Ref. [92] for how this is handled). We will assume that this is not the case, and 
that the expansion indeed is valid. Also, the effect of the wave's magnetic fields has been neglected in (2.37), since the magnetic Lorentz force on a charged particle generally is much weaker than the electric Coulomb force.

The components of the polarization vectors are generally dependent on all components of the electric field. To account for this, the $\chi^{(n)}$ are not scalars, but tensors, and the products EE, EEE, etc are tensor products. To show what Eq. (2.37) means more explicitly, let us write the electric and polarization fields as

$$
\mathbf{E}=\frac{1}{2} \sum_{j} \mathbf{E}\left(\omega_{j}\right) e^{-i \omega_{j} t}+c . c .
$$

and

$$
\mathbf{P}=\frac{1}{2} \sum_{j^{\prime}} \mathbf{P}\left(\omega_{j^{\prime}}\right) e^{-i \omega_{j^{\prime}} t}+\text { c.c. }
$$

respectively. The different summation indices are used to indicate that it is not necessarily the same frequencies in the two expansions, as we will see. If we focus on the second term in the sum of Eq. (2.37), the product between two (frequency and polarization) components of the electric field, $E_{a}\left(\omega_{j}\right) e^{-i \omega_{j} t}$ and $E_{b}\left(\omega_{k}\right) e^{-i \omega_{k} t}$, results in $E_{a}\left(\omega_{j}\right) E_{b}\left(\omega_{k}\right) e^{-i\left(\omega_{j}+\omega_{k}\right) t}$. A coupling between these two components can thus produce an oscillation in the polarization field with angular frequency $\omega_{j}+\omega_{k}$. Writing out one component of the second-order nonlinear contribution to $\mathbf{P}(\omega)$ gives

$$
P_{a}^{(2)}\left(\omega=\omega_{j}+\omega_{k}\right)=\sum_{b, c} \sum_{(j, k)} \epsilon_{0} \chi_{a, b, c}^{(2)}\left(\omega ; \omega_{j}, \omega_{k}\right) E_{b}\left(\omega_{j}\right) E_{c}\left(\omega_{k}\right),
$$

where $a, b, c=\{x, y, z\}$, and the sum over $j$ and $k$ runs over all pairs of frequencies for which $\omega=\omega_{j}+\omega_{k}$. This equation defines the components of the secondorder susceptibility tensor $\chi^{(2)}$, and it can be generalized to higher-order nonlinear susceptibilities in a straightforward way. If the material is lossless the susceptibility tensors are real, otherwise they are complex- valued [92].

The physical mechanism behind the polarization that gives rise to the optical Kerr effect is a distortion of the electron cloud around the atoms. It turns out that it is necessary to have very high-intensity light in this case to observe any nonlinear response from the material, which is typically only attainable with lasers ${ }^{10}$.

The $n$-th order susceptibility tensor $\chi^{(n)}$ is of rank $(n+1)$ and has in total $3^{n+1}$ components, but if the material possesses certain symmetries, many of them are either related to each other, or zero. For an isotropic material all even-order nonlinear susceptibilities vanish ${ }^{11}$, meaning that the lowest-order nonlinear contribution is third order. It also turns out that the 81 components $\chi^{3}$ can be expressed

\footnotetext{
${ }^{10}$ The starting point of nonlinear optics is by many considered to be the demonstration of second-harmonic generation in 1961 [93], which was only the year after the first working laser was built by Maiman [94]. Second-harmonic generation is when part of the incident light undergoes a frequency doubling, and the mechanism behind this can be understood from Eq. (2.40), where the polarization will have a component with frequency $2 \omega_{j}$ if $\omega_{j}=\omega_{k}$, which in its turn can generate radiation with this doubled frequency.

${ }^{11}$ It is actually only necessary that the material is centro-symmetric for this [92].
} 
with only three independent non-zero components (only components $\chi_{a b c d}^{3}$ where the indices are equal in pairs are nonzero, e.g. $\chi_{x x y y}^{3}, \chi_{x y x y}^{3}$, and $\left.\chi_{x x x x}^{3}\right)$ [92].

Let us assume that an isotropic sample is irradiated with linearly polarized (in $\hat{\mathbf{x}}$-direction) and monochromatic light, i.e.,

$$
\mathbf{E}=\frac{1}{2}\left(E e^{-i \omega t}+E^{*} e^{i \omega t}\right) \hat{\mathbf{x}}
$$

and that we only have to account for the linear and third-order nonlinear susceptibility. In this case only one component of $\chi^{(3)}$ has to be included in (2.37), namely $\chi_{x x x x}^{(3)}$, since the electric field cannot produce a polarization in any other direction than $x$ without breaking the isotropic symmetry (no reason that it should point in $\hat{\mathbf{y}}$ rather than $-\hat{\mathbf{y}}$ for example). By inserting (2.41) into (2.37) under these assumptions, we get

$$
\mathbf{P}=\epsilon_{0} \chi^{(1)} \mathbf{E}+\frac{3 \epsilon_{0} \chi^{(3)}}{4}|E|^{2} \mathbf{E}+\frac{\epsilon_{0} \chi^{(3)}}{8}\left(E^{3} e^{-3 i \omega t}+\left(E^{*}\right)^{3} e^{3 i \omega t}\right) \hat{\mathbf{x}}
$$

The first two terms on the right-hand side have the same frequency as the incident light, and are the ones of relevance for the refractive index. One may thus introduce an effective susceptibility

$$
\chi_{e}=\chi^{(1)}+\frac{3 \chi^{(3)}}{4}|E|^{2} .
$$

For nonmagnetic material $n^{2}=1+\chi_{e}$, so

$$
n=\sqrt{1+\chi^{(1)}+\frac{3 \chi^{(3)}}{4}|E|^{2}} \approx n_{0}+n_{2}|E|^{2},
$$

where $n_{0}=\sqrt{1+\chi^{(1)}}$ and $n_{2}=3 \chi^{(3)} / 8 n_{0}$. We have thus obtained the same expression as Eq. (2.36), i.e. the optical Kerr effect!

\subsubsection{Nonlinear Waveguide Arrays}

Our interest is not so much in single waveguides as in arrays of nonlinear dielectric waveguides. These are assumed to be sufficiently close to each other that the evanescent fields couple the guiding modes of the different waveguides ${ }^{12}$, so that light can be transferred between the waveguides. We will use coupled mode theory in Sec. 3.3 to show that this setting, under certain conditions, can be described with DNLS models.

There are several experimental schemes available that can realize arrays of nonlinear waveguides, of which we now mention a few. The waveguides can be etched onto a semiconductor substrate, a common choice being AlGaAs, where a layer of $\mathrm{Al}_{0.18} \mathrm{Ga}_{0.82} \mathrm{As}$ is used as core material, which is sandwiched between two cladding layers of $\mathrm{Al}_{0.24} \mathrm{Ga}_{0.76}$ As. Ridges are etched into the top cladding layer,

\footnotetext{
${ }^{12}$ Often only the nearest-neighbor coupling is important, but there are exceptions. For example, in square lattices the diagonal coupling may also become important [95].
} 
which cause a refractive index change in the core material [96]. The manufacturing process restricts this type of waveguide array to planar configurations, e.g. lattices in one (transverse) dimension.

Nonlinear waveguides can also be optically induced in photorefractive crystals [97]. By using lasers to create standing waves inside the crystal, the varying electric field intensity then induces a periodically varying refractive index profile, which effectively acts as a waveguide array for an additional light beam (the probe beam). This method exploits the large electro-optic anisotropy of certain photo-refractive crystals (often strontium barium niobate crystals). This makes it possible for the inducing beam, which is polarized in a particular direction, to propagate almost without any nonlinear response and diffraction, whereas the probe beam, which is polarized in a different direction, feels a strong nonlinear response from the crystal. One advantage of this scheme is the ability to change the system parameters in real time, e.g. by varying the intensity of the lasers, and that the magnitude and sign of the nonlinearity can be controlled with an external bias voltage.

A third way to create waveguides is to 'write' them in a material (e.g. fused silica) with high-intensity, femtosecond lasers [98]. The lasers will induce a structural change in the material at the laser's focal point, causing a permanent change of refractive index. The advantage of this technique is that waveguides are permanent and insensitive to the real time conditions. This is also a very versatile method regarding the geometry of both the waveguides and the arrays.

\section{Discrete Breathers}

The possibility to generate $\mathrm{DBs}^{13}$ was originally proposed by Christodoulides and Joseph in 1988 [99]. The first experimental report of DBs in optical waveguides was for a one-dimensional lattice of ridge waveguides etched onto an AlGaAs substrate [100]. Since then, DBs in one-dimensional arrays have been observed both with waveguides that have been optically induced in photorefractive crystals [101] and with waveguides written with femtosecond lasers [102]. The first report of DBs in a two-dimensional lattice was for optically induced waveguides in a photorefractive crystal [103], but it has since also been realized with femtosecond laser-written waveguides [104].

Since the first demonstrations of DBs with the various experimental techniques, there have been many additional studies of various types of DBs and properties (see Refs. $[105,106]$ and references therein). For example, after the first demonstration of DBs in AlGaAs waveguides [100], the dynamics and mobility of DBs were studied with the same experimental setup [107].

\section{Vortices}

Discrete vortex solitons (DVSs) have been studied quite extensively with optically induced waveguides in photorefractive crystals. The first reported experimental realizations were of DVSs with topological charge one in two-dimensional square

\footnotetext{
${ }^{13}$ The terminology 'discrete soliton' is often used in this context, as one does not measure the oscillating electric field but an averaged intensity.
} 
lattices $[108,109]$. Further experimental studies in two-dimensional square lattices include DVSs that bifurcate from the second linear band [110,111], and with topological charge equal to two [112]. DVSs have also been observed in hexagonal lattices, including discrete multivortex solitons (several vortices inside the cluster of excited waveguides) [113], DVSs with topological charge two [114], and single DVSs [115]. Some of the observed discrete vortex solitons in Refs. [112,115] are charge flipping, which is caused by anisotropies in the lattices (the phase of the light at different sites will increase at different rates), and it is shown in [115] that by stretching the hexagonal lattice, the charge flipping can be changed and even suppressed.

More recently, discrete vortices were also observed in a four-site (quadratic) waveguide structure, where each waveguide was optically induced with a Bessel beam [116]. This technique makes it possible to create more arbitrary waveguide structures than the extended lattice types discussed above. It was also shown that the charge flipping of these discrete vortices can be controlled with the intensity of the probe beam. 


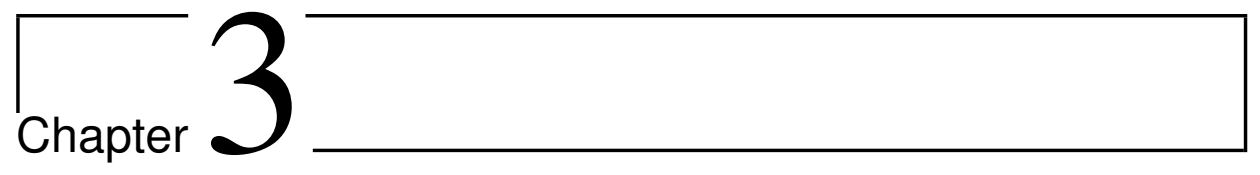

\section{Discrete Nonlinear Schrödinger Equation}

\subsection{General Properties}

The discrete nonlinear Schrödinger (DNLS) equation is just like many of the equations we encountered in chapter 1 generic, and arises in contexts when lowest order effects of nonlinearity and lattice dispersion are accounted for [117]. Early applications of the DNLS model include polarons in molecular crystals [118], energy transport in proteins [119] and vibrational modes in small molecules such as benzene [120], while examples of more recent are optical waveguide arrays and BECs in optical lattices (see chapter 2). The arguably most common version of the DNLS equation is

$$
i \frac{\mathrm{d} \psi_{\mathbf{R}}}{\mathrm{d} t}+\delta \sum_{<\mathbf{R}^{\prime}>} \psi_{\mathbf{R}^{\prime}}+\gamma\left|\psi_{\mathbf{R}}\right|^{2} \psi_{\mathbf{R}}=0
$$

where $\psi_{\mathbf{R}}$ is the complex amplitude of site $\mathbf{R}, \gamma$ is the on-site nonlinearity parameter, $\delta$ is the nearest-neighbor coupling parameter, and the sum is thus running over the nearest neighbors. The model can in principle be of any dimension, and the number of sites may be either finite and infinite. The work presented in papers I-VI are all concerned with rather small lattices, where Papers I-IV and VI deal with DNLS-rings ${ }^{1}$, i.e. one-dimensional periodic models (see Fig. 3.1(a)-(b)), while in Paper $\mathrm{V}$ we also add an extra site in the middle of the ring (see Fig. 3.1(c)). The discussion in this section will therefore be more focused on one-dimensional

\footnotetext{
${ }^{1}$ Papers I-III and VI are not primarily focused on DNLS models, but on quantum signatures in Bose-Hubbard models of solutions to DNLS models. Also, Papers I and III study lattice compactons (see Sec. 3.7.2) which are exact solutions for all rings larger than the compacton, but the work is focused on four-site rings, due to computational limitations.
} 

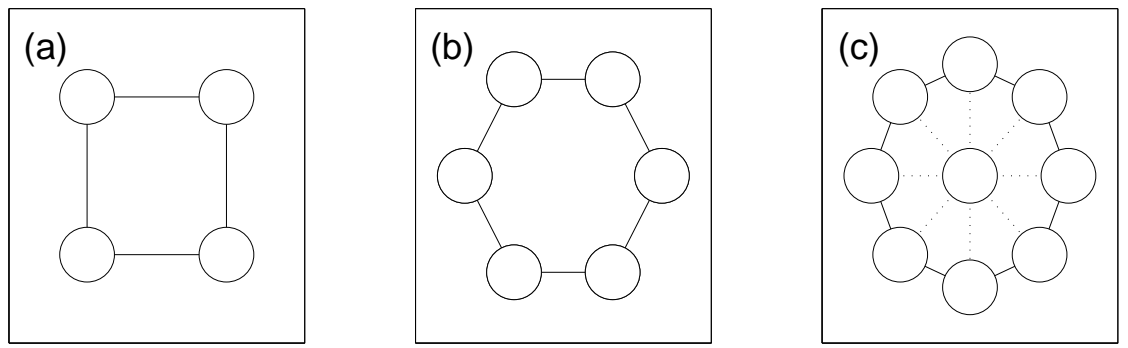

Figure 3.1. (a)-(b) Illustration of some different site configurations studied in Papers I-IV and VI, focusing on rings of different sizes. (c) In Paper V, the ring sites are also coupled to an additional central site.

models, which have the form

$$
i \frac{\mathrm{d} \psi_{j}}{\mathrm{~d} t}+\delta\left(\psi_{j+1}+\psi_{j-1}\right)+\gamma\left|\psi_{j}\right|^{2} \psi_{j}=0
$$

For finite one-dimensional lattices, we denote the number of sites with $L$. In analogy with the continuous NLS equation, one can use the term 'DNLS' for a more general class of equations, but it is not uncommon that 'the DNLS equation' means Eq. (3.1) with cubic nonlinearity, a convention we will adopt throughout this chapter.

Equation (3.1) can be derived from the Hamiltonian,

$$
H=-\delta \sum_{<\mathbf{R}, \mathbf{R}^{\prime}>} \psi_{\mathbf{R}^{\prime}} \psi_{\mathbf{R}}^{*}-\frac{\gamma}{2} \sum_{\mathbf{R}}\left|\psi_{\mathbf{R}}\right|^{4},
$$

where $<\mathbf{R}, \mathbf{R}^{\prime}>$ indicate summation over neighbor pairs, and $\psi_{\mathbf{R}}$ and $i \psi_{\mathbf{R}}^{*}$ take the role of generalized coordinates and momenta, respectively ${ }^{2}$. Equation (3.1), and its complex conjugate, are thus given by

$$
\begin{aligned}
\frac{d \Psi_{\mathbf{R}}}{d t} & =\frac{\partial H}{\partial\left(i \Psi_{\mathbf{R}}^{*}\right)}, \\
i \frac{d \Psi_{\mathbf{R}}^{*}}{d t} & =-\frac{\partial H}{\partial \Psi_{\mathbf{R}}} .
\end{aligned}
$$

The Hamiltonian is a conserved quantity, which is related (through Noether's theorem [59]) to the time-invariance of the model. The DNLS model also has a second conserved quantity, namely the (total) norm

$$
\mathcal{N}=\sum_{\mathbf{R}}\left|\Psi_{\mathbf{R}}\right|^{2}
$$

\footnotetext{
${ }^{2}$ The $\psi_{\mathbf{R}}$ and $i \psi_{\mathbf{R}}^{*}$ are strictly not independent variables, but one may instead use the real and imaginary part of $\psi_{\mathbf{R}}$ as canonical variables, which gives the same result.
} 
This conservation law is instead connected to the model's global gauge invariance, $\psi_{\mathbf{R}} \rightarrow e^{i \alpha} \psi_{\mathbf{R}}, \alpha \in \mathbb{R}$. The conservation of norm will for BECs correspond to a conservation of particles, while it for optical waveguides corresponds to a conservation of Poynting power. Since there are two conserved quantities, the DNLS model with two sites (the dimer) is integrable, and it can be completely solved in terms of elliptic functions. Systems with more sites are however non-integrable [117].

The DNLS model used in Paper V also has an extra site in the middle of the ring,

$$
\begin{aligned}
i \frac{\mathrm{d} \psi_{j}}{\mathrm{~d} t}+\delta\left(\psi_{j+1}+\psi_{j-1}\right)+\delta_{C} \psi_{C}+\gamma\left|\psi_{j}\right|^{2} \psi_{j} & =0 \\
i \frac{\mathrm{d} \psi_{C}}{\mathrm{~d} t}+\delta_{C} \sum_{j=1}^{L} \psi_{j}+\gamma_{C}\left|\psi_{C}\right|^{2} \psi_{C} & =0
\end{aligned}
$$

where $j=1, \ldots, L$ (the number of ring-sites), $\psi_{C}$ is the complex field of the central cite, $\delta_{C}$ is the coupling parameter between a ring-site and the central site, and $\gamma_{C}$ is the nonlinearity parameter for the central site. This model also has a conserved norm, and can be derived from a (conserved) Hamiltonian, which is similar to (3.3), but where $\delta$ and $\gamma$ should be replaced with $\delta_{C}$ and $\gamma_{C}$ for terms associated with the central site.

A continuity equation for the norm of a single site can readily be derived from Eq. (3.1):

$$
\frac{\mathrm{d}\left|\psi_{\mathbf{R}}\right|^{2}}{\mathrm{~d} t}=\sum_{\mathbf{R}^{\prime}} J_{\mathbf{R}^{\prime}, \mathbf{R}}
$$

where

$$
J_{\mathbf{R}^{\prime}, \mathbf{R}}=2 \delta \operatorname{Im}\left(\psi_{\mathbf{R}^{\prime}}^{*} \psi_{\mathbf{R}}\right)
$$

is the current from the neighboring site $\mathbf{R}^{\prime}$ to site $\mathbf{R}$. By writing $\psi_{\mathbf{R}}=\left|\psi_{\mathbf{R}}\right| \exp \left(i \theta_{\mathbf{R}}\right)$, the current can also be written as

$$
J_{\mathbf{R}^{\prime}, \mathbf{R}}=2 \delta\left|\psi_{\mathbf{R}^{\prime}}\right|\left|\psi_{\mathbf{R}}\right| \sin \left(\theta_{\mathbf{R}}-\theta_{\mathbf{R}^{\prime}}\right),
$$

clearly showing that the current between two sites depends on the difference in phase between them, as well as their amplitudes.

The DNLS model possesses a specific type of stationary solutions,

$$
\psi_{\mathbf{R}}(t)=\phi_{\mathbf{R}} e^{i \omega_{0} t}
$$

where $\phi_{\mathbf{R}}$ is a time-independent site-amplitude. Plugging (3.10) into Eq. (3.1) yields that the amplitudes are solutions to the algebraic equation

$$
\delta \sum_{<\mathbf{R}^{\prime}>} \phi_{\mathbf{R}^{\prime}}+\gamma\left|\phi_{\mathbf{R}}\right|^{2} \phi_{\mathbf{R}}-\omega_{0} \phi_{\mathbf{R}}=0,
$$

which is the equation one in practice solves if stationary solutions are the ones of interest. 
Another type of solutions we will be concerned with are those which are (generally) quasi-periodic, but become periodic in a co-rotating frame:

$$
\psi_{\mathbf{R}}(t)=\phi_{\mathbf{R}}(t) e^{i \omega_{0} t}
$$

where all $\phi_{\mathbf{R}}(t)=\phi_{\mathbf{R}}(t+T)$. This type of solution instead becomes a periodic solution to the equation

$$
i \frac{\mathrm{d} \phi_{\mathbf{R}}}{\mathrm{d} t}+\delta \sum_{<\mathbf{R}^{\prime}>} \phi_{\mathbf{R}^{\prime}}+\gamma\left|\phi_{\mathbf{R}}\right|^{2} \phi_{\mathbf{R}}-\omega_{0} \phi_{\mathbf{R}}=0 .
$$

The two conserved quantities reduce the dimension of the parameter space of independent solutions. For solutions with finite norm, which always is the case for finite lattices, a global rescaling of the site amplitudes can be performed to give the solution a norm with a chosen value. This must however be accompanied with an appropriate transformation of the parameters $\delta$ and $\gamma$. One may thus, without loss of generality, make a restriction to solutions with this particular norm. It is however possible to instead let the norm take any value, and instead restrict the value of either $\gamma$ or $\delta$. Furthermore, the time-variable $t$ can be rescaled to restrict the value of one additional parameter. For stationary solutions, this implies that the value of two of the following four parameters can be fixed: $\gamma, \delta, \mathcal{N}, \omega_{0}\left(\omega_{0}\right.$ is affected by a rescaling of $t$ ). Quasiperiodic solutions of type (3.12) are also characterized by the period $T$ in the co-rotating frame.

Furthermore, changing the sign on both $\gamma$ and $\delta$ in Eq. (3.1) will effectively be the same as changing the sign of $t$, which does not change the character of the solution. Note especially that this transformation does not make a stable solution unstable or vice versa, since the model has a time-reversal symmetry. This transformation will however flip the energy scale upside down. It is in some lattices also possible to make a staggering transformation, where the sign of every other site is changed. In DNLS-rings this transformation corresponds to $\psi_{j} \rightarrow(-1)^{j} \psi_{j}$, which only works if there is an even number of sites. In higher dimensions, the validity of a staggering transformation depends on which type of lattice that is considered. It works e.g. in separable lattices (e.g. the square lattice) but not in e.g. hexagonal lattices and kagomé lattices (see Fig. 1.3(c)). A staggering transformation together with $\delta \rightarrow-\delta$, or together with $(\gamma, t) \rightarrow(-\gamma,-t)$, will not change Eq. (3.1). The latter transformation flips the energy scale, which the former does not. All in all, these transformations can be used to greatly reduce the parameter space that needs to be explored, but note that one should be a bit careful if the interest is in specific energy states, e.g. the ground state.

\subsubsection{Linear Stability}

The linear stability of both stationary solutions and periodic solutions of type (3.12) can be studied by perturbing the solution in the following way: $\psi_{\mathbf{R}}^{p}(t)=$ $\left(\phi_{\mathbf{R}}(t)+\delta \phi_{\mathbf{R}}(t)\right) e^{i \omega_{0} t}$, i.e. in the co-rotating frame. For a one-dimensional system, plugging this expression into Eq. (3.2), and neglecting higher-order terms in $\delta \phi$, 
yields

$$
\begin{gathered}
\dot{\xi}_{j}=-\delta\left(\eta_{j+1}+\eta_{j-1}\right)-\gamma\left[\left(x_{j}^{2}+3 y_{j}^{2}\right) \eta_{j}+2 x_{j} y_{j} \xi_{j}\right]+\omega_{0} \eta_{j}, \\
\dot{\eta}_{j}=\delta\left(\xi_{j+1}+\xi_{j-1}\right)+\gamma\left[\left(3 x_{j}^{2}+y_{j}^{2}\right) \xi_{j}+2 x_{j} y_{j} \eta_{j}\right]-\omega_{0} \xi_{j},
\end{gathered}
$$

where the solution and perturbation both have been separated into their real and imaginary parts: $\phi_{j}=x_{j}+i y_{j}, \delta \phi_{j}(t)=\xi_{j}(t)+i \eta_{j}(t)$.

For a stationary solution, all $x_{j}$ and $y_{j}$ are time-independent, meaning that Eq. (3.14) can be expressed as

$$
\left(\begin{array}{c}
\dot{\boldsymbol{\xi}}(t) \\
\dot{\boldsymbol{\eta}}(t)
\end{array}\right)=\mathbf{D f}\left(\begin{array}{c}
\boldsymbol{\xi}(t) \\
\boldsymbol{\eta}(t)
\end{array}\right),
$$

where $\mathbf{D f}$ is a $(2 L) \times(2 L)$ real, time-independent matrix explicitly given by Eq. (3.14).

Since for stationary solutions $\mathrm{d} \phi_{j} / \mathrm{d} t=0$ in a co-rotating frame (3.13), they can also be viewed as zeros to the $2 L$-dimensional real function

$$
\begin{aligned}
& f_{j}(\mathbf{x}, \mathbf{y})=-\left[\delta\left(y_{j+1}+y_{j-1}\right)+\gamma\left(x_{j}^{2}+y_{j}^{2}\right) y_{j}-\omega_{0} y_{j}\right] \\
& f_{j+L}(\mathbf{x}, \mathbf{y})=\delta\left(x_{j+1}+x_{j-1}\right)+\gamma\left(x_{j}^{2}+y_{j}^{2}\right) x_{j}-\omega_{0} x_{j}
\end{aligned}
$$

where $\phi_{j}=x_{j}+i y_{j}$. The matrix Df is thus also the functional matrix of this function (cf. Sec. 1.5.2). The eigenvalues of $\mathbf{D f}$ determine the linear stability of the solution, where it is linearly unstable unless all eigenvalues are imaginary ${ }^{3}$. There are always two eigenvalues equal to zero, which are associated with the model's gauge invariance. These eigenvalues are associated with a single eigenmode, which sometimes is called the 'phase mode', since an infinitesimal perturbation with it results in a global, infinitesimal phase rotation [61].

For a periodic solution of type (3.12), one instead considers how the small perturbation evolves after a period $T$. By integrating Eq. (3.14) for $t=0$ to $t=T$ with $2 L$ linearly independent initial conditions, one can construct

$$
\left(\begin{array}{l}
\boldsymbol{\xi}(T) \\
\boldsymbol{\eta}(T)
\end{array}\right)=\mathcal{F}_{T}\left(\begin{array}{c}
\boldsymbol{\xi}(0) \\
\boldsymbol{\eta}(0)
\end{array}\right)
$$

for an arbitrary perturbation, where $\mathcal{F}_{T}$ is the $(2 L) \times(2 L)$ Floquet matrix (cf. Sec. 1.5.2). The periodic solution is linearly unstable unless all eigenvalues (Floquet multipliers) reside on the unit circle in the complex plane. This type of periodic solution has at least four eigenvalues that are equal to unity. Two of them are associated with the gauge invariance and the phase mode, while the other two are associated with a perturbation in the velocity direction $\delta \phi=\dot{\phi}[121]$.

\subsection{DNLS and BECs in Optical Lattices}

The DNLS model can apply to BECs in deep optical lattices when there is a large number of particles in each potential well (i.e. site). Note though that the optical

\footnotetext{
${ }^{3}$ Sometimes the imaginary number $i$ is extracted from the functional matrix, so that the solution is linearly unstable unless all eigenvalues are real.
} 


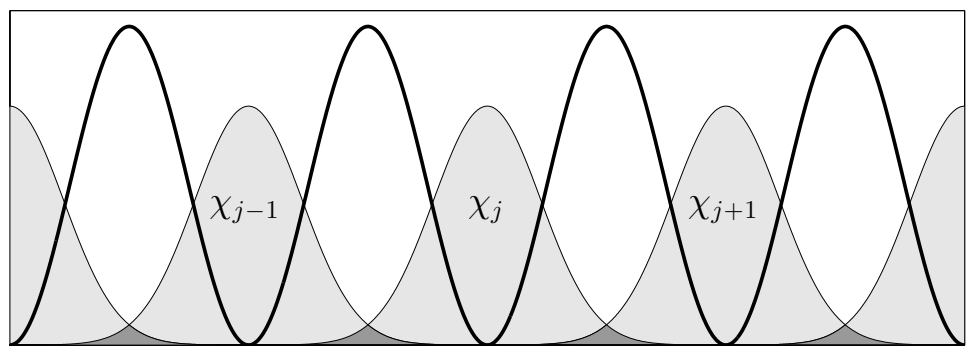

Figure 3.2. Illustration of a BEC placed in a one-dimensional optical lattice. The gray regions illustrate the individual wave-functions for the different sites.

lattice should not be too deep since there needs to be phase coherence between bosons on different sites (see discussion on superfluid-insulator crossover in Sec. 4.9). When deriving the DNLS model in this context, two different paths can be taken: either the Gross-Pitaevskii equation (2.7) is discretized, or one employs mean-field techniques on the Bose-Hubbard model. The essential difference is thus what is done first on the fundamental quantum mechanical description: the meanfield approximation or the discretization. We will in this section demonstrate how to discretize the Gross-Pitaevskii equation, but leave the mean-field treatment of the Bose-Hubbard model to Sec. 4.5.

The derivation of the DNLS model by a discretization of the Gross-Pitaevskii equation (2.7) was originally done in a paper by Trombettoni and Smerzi [122] to model an earlier experiment [123]. We will for simplicity make the derivation for one dimension, and also assume that the system is perfectly periodic in this direction, but the method can readily be generalized to higher dimensions and other more complex settings. The external potential $V(\mathbf{r})$ in the Gross-Pitaevskii equation (2.7) is thus taken to be periodic in one dimension, while it tightly confines the particles in the two transverse to this, which effectively creates a periodic row of equivalent potential wells. For sufficiently deep potential wells, the atoms are assumed to form separate condensates in each well, where each condensate is described by a wave function $\chi_{j}=\chi\left(\mathbf{r}-\mathbf{r}_{j}\right)$ which is well localized in the potential well centered at $\mathbf{r}_{j}$, meaning that $\int \chi_{i} \chi_{j} d \mathbf{r} \simeq 0$ for $i \neq j$. The wave functions are furthermore taken to be real and normalized ${ }^{4}, \int \chi_{j}^{2} d \mathbf{r}=1$. Figure 3.2 illustrates this scenario.

The macroscopic wave function $\Psi(\mathbf{r}, t)$ in the Gross-Pitaevskii equation (2.7)

\footnotetext{
${ }^{4} \mathrm{~A}$ convenient choice is Wannier functions [124], discussed in Sec. 4.2.
} 
is now expanded in terms of these localized functions:

$$
\begin{array}{r}
\Psi(\mathbf{r}, t)=\sqrt{N} \sum_{j} \psi_{j}(t) \chi\left(\mathbf{r}-\mathbf{r}_{j}\right), \\
\sum_{j}\left|\psi_{j}\right|^{2}=1,
\end{array}
$$

where $N$ is the total number of particles in the condensate. The complex quantities $\psi_{j}(t)=\sqrt{\rho_{j}(t)} e^{i \theta_{j}(t)}$, which become the variables of the DNLS model, describe the (relative) number of particles $\rho_{j}=N_{j} / N\left(N_{j}\right.$ is the absolute number of particles at site $j$ ) and phase $\theta_{j}$ of the condensate at site $j$. The DNLS model's conservation of norm is thus related to the conservation of particles in the BEC. Multiplying the Gross-Pitaevskii equation with $\chi\left(\mathbf{r}-\mathbf{r}_{j}\right)$ and integrating over all space will produce a number of different terms of the types $\psi_{a}$ and $\psi_{a} \psi_{b} \psi_{c}^{*}$, which are proportional to two different types of overlap integrals

$$
\begin{array}{r}
A_{j, a}=-\frac{1}{\hbar} \int\left[\frac{\hbar^{2}}{2 m} \nabla \chi_{j} \cdot \nabla \chi_{a}+\chi_{j} V(\mathbf{r}) \chi_{a}\right] d \mathbf{r}, \\
B_{j, a, b, c}=-\frac{U_{0} N}{\hbar} \int \chi_{j} \chi_{a} \chi_{b} \chi_{c} d \mathbf{r},
\end{array}
$$

respectively. Assuming that the wave functions $\chi_{j}$ are so well localized that all overlap integrals but the following can be neglected,

$$
\begin{array}{r}
\beta=-\frac{1}{\hbar} \int\left[\frac{\hbar^{2}}{2 m}\left(\boldsymbol{\nabla} \chi_{j}\right)^{2}+V(\mathbf{r}) \chi_{j}^{2}\right] d \mathbf{r}, \\
\delta=-\frac{1}{\hbar} \int\left[\frac{\hbar^{2}}{2 m} \nabla \chi_{j} \cdot \nabla \chi_{j \pm 1}+\chi_{j} V(\mathbf{r}) \chi_{j \pm 1}\right] d \mathbf{r} \\
\gamma=-\frac{U_{0} N}{\hbar} \int \chi_{j}^{4} d \mathbf{r},
\end{array}
$$

results in the following DNLS equation

$$
i \frac{\mathrm{d} \psi_{j}}{\mathrm{~d} t}+\beta \psi_{j}+\delta\left(\psi_{j+1}+\psi_{j-1}\right)+\gamma\left|\psi_{j}\right|^{2} \psi_{j}=0
$$

Equation (3.21) contains the extra linear on-site potential term $\beta \psi_{j}$ compared to Eq. (3.2), which can be removed by the variable substitution $\psi_{j} \rightarrow \exp (-i \beta t) \psi_{j}$. The parameters $\beta$ can however be site-dependent to account for e.g. a slowly varying trapping potential in the lattice's direction, in which case they cannot be removed with this type of transformation. The parameter $\gamma$ indicates the interaction strength between particles in the same condensate (i.e. at the same site), while $\delta$ is related to the tunneling rate of particles from a condensate at one site, to the condensate at a neighbor site. The hopping parameter $\delta$ can in principle be site-dependent, but it is often assumed that this can be neglected, which we also will do. 


\subsection{DNLS and Optical Waveguide Arrays}

The DNLS model can be derived for an optical waveguide array, modeling e.g. the experiments described in Sec. 2.2.3, by using the coupled mode theory discussed in Sec. 2.2.1. We will, analogous to the previous section, only consider a one-dimensional array of equivalent and equally spaced waveguides. The nonlinearities of these waveguides are further assumed to be of Kerr type, so that the full array's index of refraction is $n(\mathbf{r}, t)=n_{0}+n_{2}|E(\mathbf{r}, t)|^{2}$, as in Sec. 2.2.2. The total polarization of the array is thus $\mathbf{P}=\mathbf{D}-\epsilon_{0} \mathbf{E}=\left(n^{2}-1\right) \epsilon_{0} \mathbf{E}$. The 'unperturbed' system is taken to be a single waveguide with a linear index of refraction $n_{j}$, with the associated unperturbed polarization $\mathbf{P}_{0}=\left(n_{j}^{2}-1\right) \epsilon_{0} \mathbf{E}$. From (2.27), the perturbation polarization is then given by

$$
\mathbf{P}_{\text {pert }}=\mathbf{P}-\mathbf{P}_{0}=\left(n^{2}-n_{j}^{2}\right) \epsilon_{0} \mathbf{E} \simeq\left(n_{0}^{2}-n_{j}^{2}\right) \epsilon_{0} \mathbf{E}+2 n_{0} n_{2} \epsilon_{0}|E|^{2} \mathbf{E},
$$

where a second-order term in Kerr index $n_{2}$ has been neglected in the last step.

The waveguides are assumed to be sufficiently far apart that only the overlap between guiding modes of nearest-neighbor waveguides needs to be taken into account. It is also assumed that the dimensions of the waveguides and frequency $\omega$ of the incident light are chosen to permit only single-mode propagation, which we for simplicity assume is a TE mode that is linearly polarized in the $\hat{\mathbf{x}}$-direction. The electric field in waveguide $j$ is thus taken to be

$$
\mathbf{E}_{j}=\frac{1}{2}\left(\psi_{j-1} \mathcal{E}_{j-1}+\psi_{j} \mathcal{E}_{j}+\psi_{j+1} \mathcal{E}_{j+1}\right) e^{i(\beta z-\omega t)} \hat{\mathbf{x}}+\text { c.c. },
$$

where $\mathcal{E}_{j}$ is the guiding mode of the $j$-th waveguide, $\psi_{j}$ indicating its amplitude and phase, and $\mathcal{E}_{j}(\mathbf{r} \pm \mathbf{d})=\mathcal{E}_{j \mp 1}(\mathbf{r})$, where $\mathbf{d}$ is the translation vector between adjacent waveguides.

Due to the localization of the guiding modes, the quadratic term of the nonlinear index of refraction may as a first approximation be taken as (compare with Eqs. (2.41) and (2.44))

$$
\left|E_{j}\right|^{2} \approx\left|\psi_{j}\right|^{2} \mathcal{E}_{j}^{2}
$$

A perturbation polarization $\mathbf{P}_{\text {pert }}$ can now be calculated by using these $\mathbf{E}_{j}$ and $\left|E_{j}\right|^{2}$ in Eq. (3.22), which in its turn can be inserted into the coupled mode equation (2.34). By equating the terms that vary like $e^{i(\beta z-\omega t)}$, and neglecting the terms with integrands $\mathcal{E}_{j}^{3} \mathcal{E}_{j \pm 1}$, a DNLS equation like (3.21) is obtained, with

$$
\begin{array}{r}
\beta=\frac{\omega \epsilon_{0}}{4 P} \int\left(n_{0}^{2}-n_{j}^{2}\right) \mathcal{E}_{j}^{2} d x d y \\
\delta=\frac{\omega \epsilon_{0}}{4 P} \int\left(n_{0}^{2}-n_{j}^{2}\right) \mathcal{E}_{j \pm 1} \mathcal{E}_{j} d x d y \\
\gamma=\frac{\omega \epsilon_{0}}{2 P} \int n_{0} n_{2} \mathcal{E}_{j}^{4} d x d y
\end{array}
$$

and propagation distance $z$ replacing time $t$. 


\subsection{Extended Models}

As was seen in the previous Secs. 3.2 and 3.3, the derivation of the standard DNLS model for a physical system relies on a number of assumptions and approximations. There are thus regimes where it is appropriate to extend the model with additional terms.

The extended DNLS model that is of primary interest to us was originally derived in the Master thesis by A. Eriksson [125] (first published in [34]) for an optical waveguide array, and has the following form

$$
\begin{array}{r}
i \frac{\mathrm{d} \psi_{j}}{\mathrm{~d} t}+K_{1} \psi_{j}+K_{2}\left(\psi_{j+1}+\psi_{j-1}\right)+K_{3}\left|\psi_{j}\right|^{2} \psi_{j} \\
+K_{4}\left[2 \psi_{j}\left(\left|\psi_{j-1}\right|^{2}+\left|\psi_{j+1}\right|^{2}\right)+\psi_{j}^{*}\left(\psi_{j-1}^{2}+\psi_{j+1}^{2}\right)\right] \\
+K_{5}\left[2\left|\psi_{j}\right|^{2}\left(\psi_{j-1}+\psi_{j+1}\right)+\psi_{j}^{2}\left(\psi_{j-1}^{*}+\psi_{j+1}^{*}\right)\right. \\
\left.+\psi_{j-1}\left|\psi_{j-1}\right|^{2}+\psi_{j+1}\left|\psi_{j+1}\right|^{2}\right]=0 .
\end{array}
$$

The first four terms are just the standard DNLS model, with the parameters of Eq. (3.21) renamed as $K_{1}=\epsilon, K_{2}=\delta, K_{3}=\gamma$, while the additional parameters are given by

$$
\begin{gathered}
K_{4}= \begin{cases}\frac{\omega \epsilon_{0}}{2 P} \int n_{0} n_{2} \mathcal{E}_{j \pm 1}^{2} \mathcal{E}_{j}^{2} d x d y & \text { for optical waveguides, } \\
-\frac{U_{0} N}{\hbar} \int \chi_{j \pm 1}^{2} \chi_{j}^{2} d \mathbf{r} & \text { for BECs, }\end{cases} \\
K_{5}= \begin{cases}\frac{\omega \epsilon_{0}}{2 P} \int n_{0} n_{2} \mathcal{E}_{j \pm 1} \mathcal{E}_{j}^{3} d x d y & \text { for optical waveguides } \\
-\frac{U_{0} N}{\hbar} \int \chi_{j \pm 1} \chi_{j}^{3} d \mathbf{r} & \text { for BECs. }\end{cases}
\end{gathered}
$$

This model also accounts for nonlinear interactions between the neighboring guiding modes/condensates. This model was derived for optical waveguides with the same method as in Sec. 3.3, with the difference that nearest-neighbor coupling is included in Eq. (3.24),

$$
\begin{array}{r}
\left|E_{j}\right|^{2} \approx\left|\psi_{j-1}\right|^{2} \mathcal{E}_{j-1}^{2}+\left|\psi_{j}\right|^{2} \mathcal{E}_{j}^{2}+\left|\psi_{j+1}\right|^{2} \mathcal{E}_{j+1}^{2} \\
+\left(\psi_{j-1} \psi_{j}^{*}+\psi_{j-1}^{*} \psi_{j}\right) \mathcal{E}_{j-1} \mathcal{E}_{j}+\left(\psi_{j+1} \psi_{j}^{*}+\psi_{j+1}^{*} \psi_{j}\right) \mathcal{E}_{j+1} \mathcal{E}_{j} .
\end{array}
$$

Also, terms which are proportional to integrals that contain $\mathcal{E}_{j-1}^{2} \mathcal{E}_{j+1}^{2}$ are neglected. For BECs in optical lattices, $K_{4}$ and $K_{5}$ are given by Eq. (3.19b) with the appropriate values of $a, b$, and $c$ [124]. This model also conserves the norm and Hamiltonian, where the latter is given by

$$
\begin{array}{r}
H=-\sum_{j}\left[K_{1}\left|\psi_{j}\right|^{2}+K_{2} \psi_{j}\left(\psi_{j-1}^{*}+\psi_{j+1}^{*}\right)+\frac{K_{3}}{2}\left|\psi_{j}\right|^{4}\right. \\
+\frac{K_{4}}{2}\left[4\left|\psi_{j}\right|^{2}\left|\psi_{j+1}\right|^{2}+\psi_{j}^{2}\left(\psi_{j+1}^{* 2}+\psi_{j-1}^{* 2}\right)\right] \\
\left.+K_{5}\left[\psi_{j} \psi_{j+1}\left(\psi_{j}^{* 2}+\psi_{j+1}^{* 2}\right)+\psi_{j}^{*} \psi_{j+1}^{*}\left(\psi_{j}^{2}+\psi_{j+1}^{2}\right)\right]\right] .
\end{array}
$$


Some interesting properties of this model will be discussed later in this chapter, and the connection to the corresponding extended Bose-Hubbard model is discussed in Sec. 4.5.

\subsection{Numerical Methods}

The DNLS model becomes non-integrable for more than two sites, and it is therefore often necessary to use numerical methods to find the solutions of interest. These numerical methods have been used in Papers IV and V to trace out continuous families of solutions as functions of the system parameters. We will again for simplicity conduct the discussion for a one-dimensional model of equivalent sites, but generalizations to higher dimensions and more complicated settings are straightforward.

\section{Stationary Solutions}

As stated in Sec. 3.1.1, stationary solutions of type (3.10) can be viewed as zeros to the real-valued Eq. (3.16). A multi-dimensional Newton-Raphson algorithm may therefore be used when attempting to find a certain type of solution. The basic idea of the Newton-Raphson algorithm is to iterate the mapping

$$
\left(\begin{array}{l}
\mathbf{x}^{(l+1)} \\
\mathbf{y}^{(l+1)}
\end{array}\right)=\left(\begin{array}{l}
\mathbf{x}^{(l)} \\
\mathbf{y}^{(l)}
\end{array}\right)-(\mathbf{D f})^{-1} \mathbf{f}\left(\mathbf{x}^{(l)}, \mathbf{y}^{(l)}\right)
$$

with Df defined by (3.14)-(3.15), until a solution with a desired numerical accuracy has been obtained (in Paper IV we used $\sqrt{\sum_{j} f_{j}\left(\mathbf{x}^{(l)}, \mathbf{y}^{(l)}\right)^{2}}<10^{-12}$ as a convergence criterion). Index $l$ denotes iterations and $\left(\boldsymbol{x}^{(0)}, \boldsymbol{y}^{(0)}\right)^{T}$ is the initial guess of a solution which must be sufficiently close to the actual solution in order for the Newton-Raphson algorithm to converge properly.

If the Newton-Raphson algorithm is used directly on just (3.16), the norm of the obtained solution is not specified beforehand. However, by adding the extra function

$$
f_{2 L+1}\left(x_{1}, \ldots, x_{L}, y_{1}, \ldots, y_{L}\right)=\sum_{j=1}^{L}\left|x_{j}+i y_{j}\right|^{2}-\mathcal{N}_{d},
$$

the Newton-Raphson will search for solutions with this specific norm $\mathcal{N}_{d}$. The addition of an equation requires that one of the parameters $\delta, \gamma$, or $\omega_{0}$ is treated as a variable in the Newton-Raphson algorithm, meaning that it is not specified beforehand but by the Newton-Raphson algorithm. The Jacobian matrix Df is now a $(2 L+1) \times(2 L+1)$ matrix, with the extra column defined by $\mathbf{D f}_{j, 2 L+1}=$ $\partial f_{j} / \partial \zeta$, where $\zeta$ is the parameter chosen to act as a variable.

As discussed in Sec. 3.1.1, the gauge invariance of the DNLS model implies that the matrix Df has at least two eigenvalues which are zero, making it noninvertible. For those stationary solutions which do not carry any currents, one can generally specify that the solution is real, which explicitly breaks the gauge symmetry. 
As described in Sec. 1.3.2, the Newton-Raphson method can be used to trace out continuous families of solutions. Assume that a solution $\phi=\mathbf{x}+i \mathbf{y}$ has been found for a set of parameters $\delta, \gamma, \omega_{0}, \mathcal{N}$. By slightly adjusting either one or several of these parameters (one of them acts as a variable), the solution can be used as the initial guess in the Newton-Raphson algorithm, in an attempt to find a solution in the same family for these new parameter values. It is crucial that the parameters are not changed too much, since the Newton-Raphson algorithm then may find a completely different type of solution. By iterating this process of adjusting the parameters and using the old solution as the initial guess, one may trace out continuous families of solutions as functions of the parameters. As was discussed in Sec. 3.1, due to the two conserved quantities two of the four parameters $\delta, \gamma, \omega_{0}$, and $\mathcal{N}$ can be held fixed when tracing out a family of solutions. One may thus adjust one of the remaining parameters in small steps, and use the other as the variable ${ }^{5}$.

When tracing out a family of solutions with the Newton-Raphson method, it is of course necessary to have a solution in this family to begin with. This may sometimes be obtained as an analytical solution in a certain parameter limit, which we will see an example of in Sec. 3.7 when discussing discrete breathers. We will also see in Sec. 3.8 that one can find periodic solutions (of the type discussed below) by perturbing a stationary solution.

\section{Periodic Solutions}

Periodic solutions of type (3.12) can on the other hand be considered as zeros to the mapping

$$
\begin{gathered}
g_{j}^{\left(\omega_{0}, T\right)}\left(\phi_{1}(0), \ldots, \phi_{L}(0)\right)=x_{j}(T)-x_{j}(0), \\
g_{j+L}^{\left(\omega_{0}, T\right)}\left(\phi_{1}(0), \ldots, \phi_{L}(0)\right)=y_{j}(T)-y_{j}(0),
\end{gathered}
$$

where $x_{j}(T)$ and $y_{j}(T)$ have been obtained by integrating Eq. (3.2). Equation (3.32) is thus generally dependent on all $x_{j}(0)$ and $y_{j}(0)$ through the integration procedure. The machinery discussed above for finding stationary solutions and their families is, with a few adjustments, valid also for these periodic solutions. One needs to account for that the periodic solution is characterized by an additional parameter, the period $T$. Also, the matrix which is inverted in the NewtonRaphson algorithm is now $\mathcal{F}_{T}-\mathbf{I}$, where $\mathcal{F}_{T}$ is the Floquet matrix (3.17) and $\mathbf{I}$ the identity matrix. The Floquet matrix has (at least) four eigenvalues that are equal to unity (see Sec. 3.1.1), and to invert $\mathcal{F}_{T}-\mathbf{I}$, one may use a singular value decomposition [47].

\footnotetext{
${ }^{5}$ It is not necessary that the continuous families are single-valued functions of a parameter, an example being the DBs studied in Paper V. One may then need to alternate between which parameter that is treated as a variable, and which that is adjusted in small steps.
} 


\subsection{Instabilities}

There are a number of different types of instabilities that solutions of the DNLS model can experience, but we will only name a few of the most important ones for one-dimensional lattices.

The first one is the modulational instability, which occurs for plane waves $\psi_{j}=$ $\psi_{0} e^{i\left(q j-\omega_{0} t\right)}$, where they become linearly unstable to periodic modulations of the amplitude and phase. By executing a linear stability analysis of the plane waves $[126,127]$, they turn out to be modulationally unstable if the following quantity is negative:

$$
2 \delta^{2} \sin ^{2}\left(\frac{Q}{2}\right) \cos ^{2}(q)\left[2 \sin ^{2}\left(\frac{Q}{2}\right)-\frac{\gamma \psi_{0}^{2}}{\delta \cos (q)}\right]
$$

where $Q$ is the wave number of the perturbing modulation. This expression depends on both the amplitude and wave number of the plane wave, as well as the modulation's wave number, and it is positive in two cases. First, if $\delta \cos (q) / \gamma<0$, meaning that if $\delta / \gamma>0(\delta / \gamma<0)$, all plane waves with $\pi / 2<|q|<\pi(0<|q|<$ $\pi / 2)$ are linearly stable to all such perturbations. Second, if $\delta \cos (q) / \gamma>0$ while also $2 \sin ^{2}(Q / 2)>\gamma \psi_{0}^{2} / \delta \cos (q)$. All plane waves are in this case unstable to some modulations for infinite lattices. Note especially that if $\delta / \gamma>0(\delta / \gamma<0)$, all plane waves with $0<|q|<\pi / 2(\pi / 2<|q|<\pi)$ become unstable to all modulations if the amplitude of the plane wave satisfies $\psi_{0}>\sqrt{2 \delta / \gamma}$.

The second example we will discuss is oscillatory instabilities for stationary solutions. These appear through Hamiltonian Hopf bifurcations, where some eigenvalues of the functional matrix get both a non-zero real and imaginary part, and a perturbation will oscillate around the stationary solution in phase space with an exponentially increasing amplitude [61] (cf. Sec. 1.5.2). Oscillatory instabilities will typically appear when the lattice has an inhomogeneous amplitude distribution, so that it is divided into sub-lattices of sites with small and large amplitudes, respectively. The instability will then appear as a result of resonances between the internal oscillations of the different sub-lattices. Examples of this kind of configuration which experience oscillatory instabilities are discrete dark solitons ('dark breathers') [128, 129], two-site localized twisted modes [130-132] (see Sec. $3.7)$, spatially periodic or quasiperiodic nonlinear standing waves $[133,134]$ and gap modes in diatomic chains $[135,136]$. Since there always is at least one pair of stability eigenvalues which are zero, associated with the phase mode, and the oscillatory instability is associated with an additional quadruplet of complex eigenvalues, the DNLS trimer is the smallest system which can experience oscillatory instabilities. Ref. [61] studied this for the so called single depleted well (SDW) solution: $\phi=\left(\phi_{1},-\phi_{1}, 0\right), \phi_{1} \in \mathbb{R}[137]$, which is oscillatory unstable in a specific parameter interval (for $\delta, \gamma>0$ ) [138].

\subsection{Discrete Breathers}

As indicated in Sec. 1.3.2, families of DBs can be followed numerically from the anti-continuous limit, which for the standard DNLS model (3.1) corresponds to 

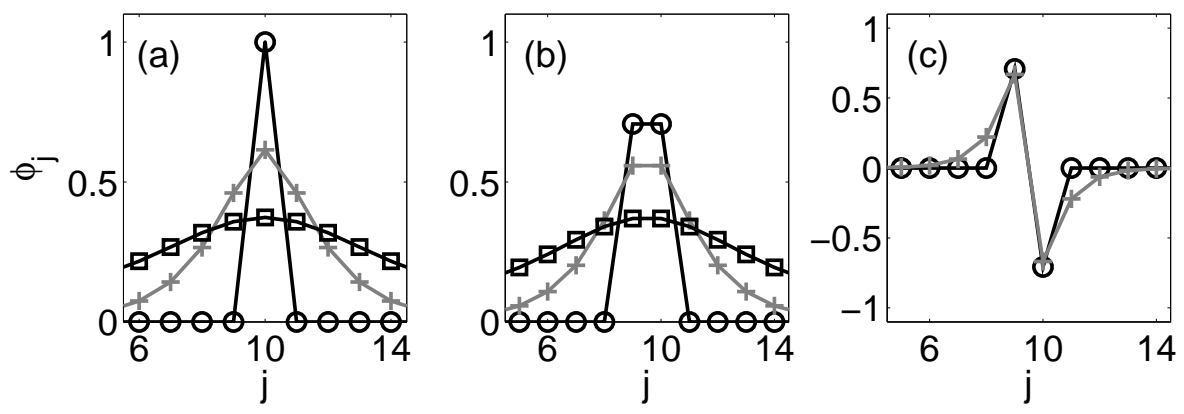

Figure 3.3. Examples of DBs in a $L=19$ site DNLS ring, with norm $\mathcal{N}=1$ and $\gamma=1$. (a)-(b) Different DBs for $\delta=0,0.4$, and 0.8. (c) Twisted localized mode for $\delta=0$ and 0.1 . Larger $\delta$ gives a more spread-out DB.

$\delta=0$. Since the sites are decoupled in this limit, one may excite some sites while keeping the others exactly zero, and it is thus trivial to create localized solutions. The simplest example of such a localized solution is when only one site is excited, which for a one-dimensional lattice corresponds to

$$
\psi_{j}(t)= \begin{cases}A e^{i \gamma A^{2} t} & \text { if } j=m \\ 0 & \text { otherwise }\end{cases}
$$

where the amplitude $A$ always can be chosen to be real due to the gauge invariance. We will have $\gamma, \delta \geq 0$ in this section, but remember that their relative sign can be changed with a staggering transformation ${ }^{6}$. In this case, (3.34) is the ground state in the anti-continuous limit for a fixed norm $\mathcal{N}$. By continuing this solution for nonzero $\delta$ with the method described in Sec. 3.5, a family of DBs which are peaked on site $m$, and symmetric around this site ('on-site symmetric') can be followed. Figure 3.3(a) shows some DBs in this family for different $\delta(\gamma=\mathcal{N}=1$ and $L=19$ ), where the DBs are more spread out for larger $\delta$. A computational advantage of studying solutions which are well localized on a part of the lattice, is that sufficiently big, finite lattices become good approximations of infinite ones.

By starting with other configurations in the anti-continuous limit, one can follow families of other types of DBs. If the interest is in stationary solutions, then all excited sites must have the same amplitude in the anti-continuous limit, since the frequencies of the excited sites increase with the amplitude (cf. Eq. (3.34)). Starting with a solution with two unequal amplitudes will generally result in a family of (quasi-)periodic solutions of type (3.12) [121].

Let us now consider stationary solutions in a one-dimensional lattice with two neighbor sites excited in the anti-continuous limit. These two sites may be either in phase or anti-phase with each other, as a different phase difference would induce

\footnotetext{
${ }^{6}$ The staggering transformation discussed in Sec. 3.1 is approximately valid also for odd $L$ if the solution is sufficiently well localized, and it is thus possible to apply it to solutions sufficiently close to the anti-continuous limit for odd $L$.
} 
a current (3.8) between them when $\delta>0$. The in-phase case gives a family of DBs which are peaked on these original two sites and symmetric around the middle point in-between them ('inter-site symmetric'), see Fig. 3.3(b). This DB, together with the on-site symmetric above, are often considered the two fundamental DBs of the one-dimensional DNLS. This inter-site symmetric DB is sometimes referred to as the Page (P) mode [139], and the on-site symmetric as the Sievers-Takeno (ST) mode [140]. We will however refer to them as the on-site and inter-site symmetric DBs through the rest of this chapter, even though other DBs can be classified in this way too. Starting with the two neighboring sites in anti-phase instead gives a corresponding family of inter-site anti-symmetric DBs, see Fig. 3.3(c). This is sometimes called the (two-site) twisted localized mode or twisted unstaggered mode $[131,132]$. The twisted localized mode can however not be followed all the way to the continuous limit $\delta \rightarrow \infty$ [132].

The on-site symmetric DB is generally stable, while the inter-site symmetric DB is unstable [141]. The twisted localized mode DB is, for a large system, stable in the interval $0 \leq\left|\delta / \omega_{0}\right| \leq 0.146$ [132], while it experiences an oscillatory instability for $\left|\delta / \omega_{0}\right|>0.146$.

Both the on-site and inter-site symmetric DBs will converge towards the NLS soliton (1.21) as they are followed to the continuous limit in an infinite lattice [141] (not the NLS breather since this has a time-varying amplitude). The two solutions will thus become more and more similar to each other as this limit is approached (cf. Fig. 3.3(a)-(b)), but they will always preserve their own symmetry, i.e. being either on-site or inter-site symmetric (this is of course not important in the continuous limit since the sites become indistinguishable). For finite rings with $L \geq 6$, these DBs instead bifurcate with the uniform solution, $\phi_{j}=\sqrt{\mathcal{N} / L}$, at

$$
\delta_{1}(L)=\frac{\gamma \mathcal{N}}{2 L} \frac{1}{\sin ^{2}(\pi / L)} .
$$

This bifurcation is identified with the critical point below which the uniform solution is modulationally unstable $[142,143]$ (see Sec. 3.6). Indeed, Eq. (3.35) is obtained by putting expression (3.33) to zero with $q=0, \psi_{0}^{2}=\mathcal{N} / L$, and $Q=2 \pi / L$ (to maximize $\delta$ ). The on-site symmetric DB is (for $\gamma>0$ ) the ground state if $\delta<\delta_{1}(L)$, while the uniform solution becomes the ground state for $\delta>\delta_{1}(L)$.

For $L<6$ on the other hand, the bifurcation scenario is a bit more complicated, as was shown in Refs. $[142,143]$. The inter-site symmetric DB will still bifurcate with the constant amplitude solution at $\delta=\delta_{1}(L)$ of Eq. (3.35), but another onsite symmetric, single-peaked solution is created in this bifurcation (thus existing for $\left.\delta>\delta_{1}(L)\right)$. The 'original' on-site symmetric DB will on the other hand not bifurcate with the constant amplitude solution, but instead with this other on-site symmetric solution at a $\delta=\delta_{3}>\delta_{1}$. The ground state of the system will also change at a $\delta=\delta_{2}$, where $\delta_{1}<\delta_{2}<\delta_{3}$, so that the on-site symmetric DB is that ground state for $\delta<\delta_{2}$, while the constant amplitude solution is the ground state otherwise.

Let us emphasize that this self-trapping transition, when the uniform ground state becomes unstable and a stable symmetry-broken ground state appears [138, 144], can be observed already for the DNLS dimer. Above a critical value of $\delta>0$ 
(for given values of $\mathcal{N}$ and $\gamma>0$ ) there exist two stable stationary solutions, corresponding to the same amplitude on both sites but either in phase or antiphase. When $\delta$ is decreased to this critical value, a bifurcation occurs for the inphase solution which becomes unstable, and a new stable solution which breaks the permutational symmetry, i.e. has unequal amplitudes on the sites, appears [144]. We will return to this scenario in Sec. 4.6, and discuss quantum signatures of this transition.

Modulational instabilities, discussed in Sec. 3.6, are also thought to be an important mechanism in the formation of DBs in physical systems $[127,145,146]$. Basically, the modulational instability will create 'lumps' with slightly higher amplitudes that can move through the lattice. These lumps can coalesce when they collide, so that eventually a number of well-localized, high-amplitude structures (DBs) have formed in the lattice [127] (these are generally not moving as high amplitude DBs tend to have poor mobility, see Sec. 3.7.1). Furthermore, some of these DBs can become unstable, basically because the amplitudes of the DB's neighboring sites increase which effectively creates an unstable multi-frequency (quasiperiodic) DB. These DBs will decay by emitting radiation which can be absorbed by the other DBs in the vicinity to further increase their amplitude $[145,146]$.

In Paper V, we studied DB-families in DNLS-rings with an additional site in the middle (cf. Fig 3.1(c) and Eq. (3.6)). There are thus two different coupling parameters in the model: between nearest neighboring sites in the ring $(\delta)$, and between a ring-site and the central site $\left(\delta_{C}\right)$. The nonlinearity parameter $\gamma$ is fixed to one in this work, but we let the nonlinearity parameter on the central site $\gamma_{C}$ take other values. In this work we utilized the methods discussed in Sec. 3.5 to follow families of stationary DBs from the anti-continuous limit. The focus was primarily on the families of the two fundamental DB ring-modes, i.e. one and two neighboring sites in the ring excited in the anti-continuous limit, respectively. As there now are two inter-site coupling parameters, we used a two-step strategy. First, the ring-coupling $\delta$ was turned up, giving us the familiar families of DBs for just the DNLS ring. Second, for chosen values of $\delta$, we turned on the coupling to the central site $\delta_{C}$, using the frequency $\omega_{0}$ as the variable, tracing out subfamilies of DBs. It turned out that, for a given $\delta$, the DB subfamilies would sometimes not be single-valued functions of $\delta_{C}$, and we would need to alternate between using $\delta_{C}$ and $\omega_{0}$ as variables in the Newton-Raphson algorithm. Using this strategy, we could determine the properties of the DB-families, e.g. in which parameter regimes they exist and when they are stable. The DB-subfamilies undergo a number of different bifurcations for certain values of $\delta$. For example, the two fundamental DB ring-modes will belong to the same subfamily in a parameter window $\delta_{1} \leq \delta \leq \delta_{2}$, and different subfamilies otherwise.

DBs exist also in higher-dimensional lattices, and similar to the one-dimensional case, one may trace out and study the properties of continuous families of DBs, characterized by their symmetries [147]. For example, in a two-dimensional square DNLS lattice, the arguably most basic DBs are those which in the anti-continuous limit have one site, two neighboring sites, and four sites in a fundamental square excited, respectively (an analogous classification can also be carried out in three dimensions [147]). The one-site DB turns out to be linearly stable only above 
a frequency threshold (for fixed $\gamma$ and $\delta$ ), in contrast to the corresponding onedimensional DB which always is stable, while the other mentioned DBs always are linearly unstable (at least for large lattices) [148-150]. Another notable difference is that there is a norm/energy threshold ('excitation threshold') for DBs in lattices with higher dimensions than one, meaning that DBs do not exist below certain values of norm and energy [151,152].

\subsubsection{Mobility}

The DBs discussed so far have been stationary on the lattice, but a question of fundamental interest is whether or not they can be made mobile. One way to set a stationary DB into motion, is to give it a 'kick' by introducing a phase gradient [153], i.e. by transforming the stationary solution according to $\phi_{j} \rightarrow e^{i j \theta} \phi_{j}, \theta \in \mathbb{R}$.

Should the on-site symmetric DB be mobile, then we can imagine that a small phase gradient will make it move slowly across the lattice by transforming continuously between the on-site and inter-site profiles. A measure of how 'good' mobility this DB has, is the energy (Hamiltonian) difference between the on-site and inter-site DBs with the same norm - smaller energy difference meaning better mobility $[154,155]$. This is called the Peierls-Nabarro barrier, and is essentially the energy it takes to translate the DB one site, if energy losses to the rest of the lattice can be neglected. For the DNLS model (3.2), the Peierls-Nabarro barrier is generally finite. A DB that is moving through the lattice will therefore lose energy by emitting radiation in the form of low-amplitude plane waves [156].

It was found in the work by Öster et al [34] that the mobility of DBs can be greatly enhanced in the extended DNLS equation (3.26). The additional terms in the Hamiltonian (3.29) can thus, if the parameters are properly tuned, decrease the energy difference between the on-site and inter-site symmetric DB. The enhanced mobility is also associated with an exchange of stability between the two DBs $[15,157]$. For certain parameter values, the on-site symmetric DB can become unstable, while the symmetric inter-site DB becomes stable, and it is in the region where the stabilities change that the Peierls-Nabarro barrier becomes very small and the DB can then travel essentially without emitting any radiation [34]. The onsite and inter-site symmetric DBs will however generally not change the stability at the same point, but there is typically a small region were both of them are unstable and a third intermediate solution (which does not exist in the standard DNLS) is stable, or vice versa. The parameters can though be tuned so that they do exchange their stability in the same point, which also optimizes the mobility [158].

\subsubsection{Lattice Compactons}

It was also found in [34] that the extended DNLS equation (3.26) supports lattice compactons (see Sec. 1.3.4), where only certain sites are excited and the rest of the lattice has exactly zero amplitude. The basic idea is that there now are several different terms that couple the nearest neighbors, and that by properly tuning the parameters, the total coupling cancels out. For a two-site compacton, this occurs for $K_{2}=-K_{5} \mathcal{N}$, but it is possible to have lattice compactons with any number of 
excited cites. The existence of lattice compactons depends only on $K_{2}, K_{5}$ and $\mathcal{N}$, but $K_{3}$ and $K_{4}$ will influence the stability and mobility of the lattice compactons (they lose their exact compactness when moving, but it is possible to obtain very localized, moving DBs by properly tuning $K_{3}$ and $K_{4}$ [158]). In Sec. 4.6.1 we discuss our studies of the quantum versions of these lattice compactons, published in Papers I and III.

\subsection{Discrete Vortices}

Families of discrete vortex solitons can be, and have been, studied numerically in two-dimensional lattices with the method in Sec. $3.5[48,159]$. The idea is again to start in the anti-continuous limit and excite a cluster of sites, but to choose the phases on these sites so that the phase increases by a multiple of $2 \pi$ (its topological charge ' $T C$ ', see Eq. (1.26)) when the excited sites are followed in a closed loop. When the inter-site coupling is turned on, a circulating current will begin to flow, and a vortex is thus created. A simple example would be for a square lattice, where in the anti-continuous limit four sites in a square have the same nonzero amplitude, and there is a phase difference of $\pi / 2$ between neighboring, excited sites [47]. This discrete vortex soliton has a $T C$ of plus/minus one (depending on convention).

Discrete vortex solitons have been studied in several different two-dimensional DNLS lattices, e.g. square [48, 49,51,159], triangular [160], honeycomb [160], and kagomé [161], and several different types of discrete vortex solitons were found in these works. These discrete vortex solitons are not expected to be stable all the way to the continuous limit, since solitons of the two-dimensional continuous NLS model are not stable. Some of the found discrete vortex solitons are however linearly stable below a threshold value of $\delta$, while others always are linearly unstable. For example, in Ref. [49] a discrete vortex soliton was found for the square lattice with $T C=1$, which is stable for $\delta \lesssim 0.13$ (they used $\gamma=1$ and $\omega_{0}=0.32$ ).

The discrete vortex solitons studied in [49] are all stationary, meaning that currents (3.8) balance to keep the amplitudes constant. It is however possible to find (quasi-)periodic discrete vortex solitons of type (3.12), where the amplitudes vary periodically [48]. The currents are thus not balanced in this case, but will also vary periodically. These vortices can, similar to (quasi-)periodic DBs, be obtained by starting with solutions in the anti-continuous limit which have sites with different amplitudes.

Discrete vortices can also be studied in systems where the sites are arranged in a ring (and also other finite clusters of sites), see Fig. 1.4(c). A DNLS model that describes such a ring will effectively be a one-dimensional periodic model, for which the plane wave solution $\psi_{j}=\sqrt{\mathcal{N} / L} e^{i\left(2 \pi k j / L-\omega_{0} t\right)}, k \in \mathbb{Z}$, can be interpreted as a vortex with topological charge $k$.

For DNLS-rings with $L=4 l$ sites, $l \in \mathbb{Z}^{+}$, there is a simple analytical formula 

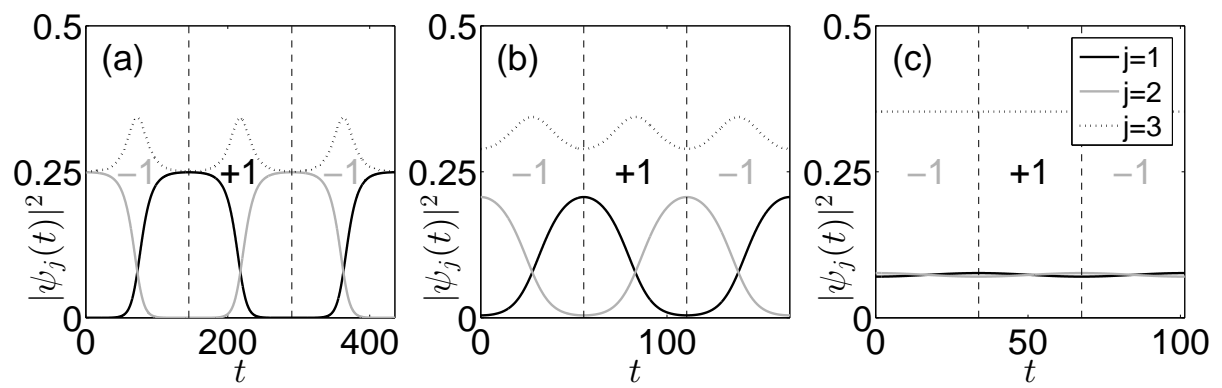

Figure 3.4. Examples of charge flipping vortices of the DNLS trimer and hexamer studied in Paper IV, all with $\mathcal{N} / L=1 / 6$. The numbers \pm 1 indicate the topological charge. (a) This charge flipping vortex is close to the SDW solution, and is essentially an unstable perturbation of it, resulting in a hole (empty site) that oscillates between two sites. (b) An intermediate charge flipping vortex in the same subfamily (with fixed norm) as the ones in (a) and (c). (c) This charge flipping vortex can be viewed as a stable perturbation of the dimerlike solution.

for charge flipping vortices [53, 162]:

$$
\begin{aligned}
\psi_{2 j-1}(t) & =(-1)^{j} a e^{i\left(\gamma a^{2} t+\alpha\right)}, \\
\psi_{2 j}(t) & =(-1)^{j} b e^{i\left(\gamma b^{2} t+\beta\right)},
\end{aligned}
$$

where $j=1, \ldots, 2 l$. This effectively creates two staggered and uncoupled sublattices, consisting of the even and odd sites, respectively. The topological charge of this solution will periodically flip between $T C= \pm l$ with the angular frequency $\gamma\left(a^{2}-b^{2}\right)$ [53]. The actual charge flipping for this solution is caused by that the phases of the different sub-lattices increase at different rates. Putting $a=b$ and $\alpha=\beta-\pi / 2$ reduces solution (3.36) to the $T C=l$ plane-wave vortex, meaning that this solution can be viewed as a charge flipping vortex of type (3.36) with an infinite flipping period. Applying an infinitesimal perturbation with the proper stability eigenmode (having eigenvalue zero) the plane-wave vortex will turn into a charge flipping one, meaning that there is no threshold in energy for reversing the direction of the current.

In Paper IV we studied charge flipping vortices in DNLS trimers and hexamers, i.e. three- and six-site rings. This work originates in the Bachelor Thesis by N. Lagerquist [163] (studying only hexamers), where the basic idea was to try to obtain charge flipping vortices by perturbing a plane-wave vortex with one of its eigenmodes (cf. the charge flipping vortices in $4 l$-rings). Charge flipping vortices were indeed found in this way, but only when applying a rather large perturbation. In Paper IV we undertook a more systematic study of these charge flipping vortices. The trimer we examined had $\gamma=1$ and $\delta=-1$, while the hexamer had $\gamma=1$ and $\delta=1$, and the solutions we studied are actually related by $\left(\psi_{1}, \psi_{2}, \psi_{3}\right) \rightarrow\left(\psi_{1},-\psi_{2}, \psi_{3},-\psi_{1}, \psi_{2},-\psi_{3}\right)$, which essentially is a repetition of the trimer solution in the hexamer, followed by a staggering transformation. The 
trimer and hexamer are thus the two smallest rings for either sign of $\delta$ which possess these types of charge flipping vortices. The main result of Paper IV is that there exist continuous families of (quasi-)periodic charge flipping vortices of type (3.12), which can be followed continuously between two stationary solutions: the SDW solution (see Sec. 3.6) and a dimerlike solution, $\left(\psi_{1},-\psi_{1}, \psi_{3}\right), \psi_{1}<\psi_{3} \in \mathbb{R}$ $[137,164]$. Close to the SDW solution, the charge flipping vortices essentially consist of a hole (empty site) which oscillates between two sites, while close to the dimerlike solution, they can be viewed as the result of (stable) perturbations added to the dimerlike solution (see Fig. 3.4). In the trimer, the charge flipping vortices are essentially always linearly stable, while for the hexamer there are regions of linear instability. The charge flipping vortices found in Ref. [163] were essentially perturbations of this type of (quasi-)periodic solutions, with some additional highfrequency 'noise' added to them. 


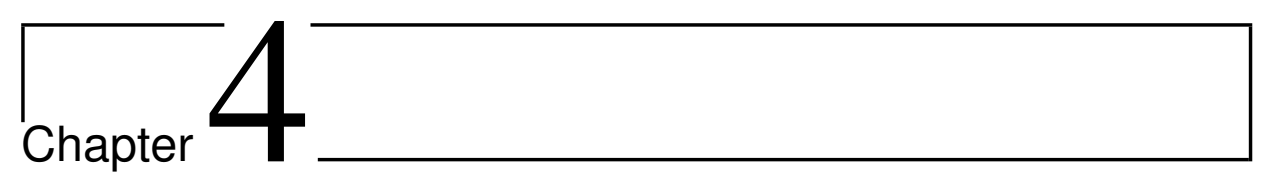

\section{Bose-Hubbard Model}

\subsection{General Properties}

Since the advent of BECs in optical lattices, the Bose-Hubbard model has received a lot of attention in this context. It is however noteworthy that it was actually studied prior to this, not at least as a quantum version of the discrete nonlinear Schrödinger equation [165-167] (cf. chapter 3), when for instance studying local modes in benzene molecules [120] and vibrations in crystals [168]. Many of these papers actually refer to the model as the 'quantum discrete nonlinear Schrödinger equation'.

The quantum mechanical Hamiltonian of the (standard) Bose-Hubbard model is given by

$$
\hat{H}=-J \sum_{<\mathbf{R}, \mathbf{R}^{\prime}>} \hat{a}_{\mathbf{R}}^{\dagger} \hat{a}_{\mathbf{R}^{\prime}}+\frac{U}{2} \sum_{\mathbf{R}} \hat{n}_{\mathbf{R}}\left(\hat{n}_{\mathbf{R}}-1\right),
$$

where hats are used to denote quantum operators, and $<\mathbf{R}, \mathbf{R}^{\prime}>$ indicates summation over neighboring sites. The model is expressed in the second quantization formalism of quantum mechanics [63], where $\hat{a}_{\mathbf{R}}$ and $\hat{a}_{\mathbf{R}}^{\dagger}$ are the bosonic annihilation and creation operators, respectively, for site $\mathbf{R}$. These obey the commutation relations

$$
\begin{array}{r}
{\left[\hat{a}_{\mathbf{R}}, \hat{a}_{\mathbf{R}^{\prime}}^{\dagger}\right]=\delta_{\mathbf{R}, \mathbf{R}^{\prime}}} \\
{\left[\hat{a}_{\mathbf{R}}, \hat{a}_{\mathbf{R}^{\prime}}\right]=\left[\hat{a}_{\mathbf{R}}^{\dagger}, \hat{a}_{\mathbf{R}^{\prime}}^{\dagger}\right]=0}
\end{array}
$$

Quantum states are in this formalism expressed in terms of Fock states,

$$
\left|\ldots, n_{\mathbf{R}^{\prime}}, n_{\mathbf{R}}, n_{\mathbf{R}^{\prime \prime}}, \ldots\right\rangle
$$

where $n_{\mathbf{R}}$ indicates the number of bosons at site $\mathbf{R}$, and the list runs over all sites in the system. The action of the creation and annihilation operator on a Fock 
state is given by

$$
\begin{gathered}
\hat{a}_{\mathbf{R}}\left|\ldots, n_{\mathbf{R}^{\prime}}, n_{\mathbf{R}}, n_{\mathbf{R}^{\prime \prime}}, \ldots\right\rangle=\sqrt{n_{\mathbf{R}}}\left|\ldots, n_{\mathbf{R}^{\prime}}, n_{\mathbf{R}}-1, n_{\mathbf{R}^{\prime \prime}}, \ldots\right\rangle, \\
\hat{a}_{\mathbf{R}}^{\dagger}\left|\ldots, n_{\mathbf{R}^{\prime}}, n_{\mathbf{R}}, n_{\mathbf{R}^{\prime \prime}}, \ldots\right\rangle=\sqrt{n_{\mathbf{R}}+1}\left|\ldots, n_{\mathbf{R}^{\prime}}, n_{\mathbf{R}}+1, n_{\mathbf{R}^{\prime \prime}}, \ldots\right\rangle .
\end{gathered}
$$

Given these expressions, a number operator can also be defined as $\hat{n}_{\mathbf{R}}=\hat{a}_{\mathbf{R}}^{\dagger} \hat{a}_{\mathbf{R}}$, for which

$$
\hat{n}_{\mathbf{R}}\left|\ldots, n_{\mathbf{R}^{\prime}}, n_{\mathbf{R}}, n_{\mathbf{R}^{\prime \prime}}, \ldots\right\rangle=n_{\mathbf{R}}\left|\ldots, n_{\mathbf{R}^{\prime}}, n_{\mathbf{R}}, n_{\mathbf{R}^{\prime \prime}}, \ldots\right\rangle
$$

Fock states are thus eigenstates of all number operators. Note that quantum states are generally not in a single Fock state, but in a superposition of many, which gives the probability of finding the particles distributed in different ways.

The first sum in Eq. (4.1) is associated with the hopping of particles between neighboring sites, and thus to the system's kinetic energy. The constant $J$ is taken to be positive, for reasons explained in Sec. 4.2. The second term on the other hand indicates the on-site interaction between particles (repulsive for $U>0$, and attractive for $U<0$ ).

The sites $\mathbf{R}$ can be distributed in any number of dimensions. The work presented in Papers I-III and VI are however focused on $L$-site Bose-Hubbard rings, where the Bose-Hubbard Hamiltonian takes the form

$$
\hat{H}=\sum_{j=1}^{L}\left[-J\left(\hat{a}_{j+1}^{\dagger} \hat{a}_{j}+\hat{a}_{j-1}^{\dagger} \hat{a}_{j}\right)+\frac{U}{2} \hat{n}_{j}\left(\hat{n}_{j}-1\right)\right],
$$

with $\hat{a}_{L+1}=\hat{a}_{1}, \hat{a}_{0}=\hat{a}_{L}$.

\subsubsection{Eigenstates}

The discussion in this section will for simplicity be conducted for one-dimensional systems, but the results hold (with the appropriate modifications) also for higher dimensions.

The Hilbert space of an $L$-site Bose-Hubbard ring is spanned by the Fock states $\left|n_{1}, \ldots, n_{L}\right\rangle$, which is infinite dimensional since all $n_{j}$ can take any positive integer value. It is however not necessary to work in the full Hilbert space; instead the dimensionality can be reduced to a finite size by considering only a fixed number of total particles

$$
N=\sum_{j=1}^{L} n_{j}
$$

This seems physically reasonable since material bosons cannot be created or destroyed (unlike photons), and is also mathematically viable since the Hamiltonian commutes with the total number operator

$$
\hat{N}=\sum_{j=1}^{L} \hat{n}_{j}
$$


This implies that energy eigenstates also are eigenstates to $\hat{N}$, i.e. have specific numbers of particles. It is hereafter assumed that we work in a subspaces with fixed total number of particles $N$.

The Hilbert space's dimension $\mathcal{D}$ is for a fixed number of particles not infinite, but given by

$$
\mathcal{D}=\frac{(N+L-1) !}{N !(L-1) !},
$$

which is the number of ways $N$ indistinguishable particles can be placed in $L$ wells. This grows quite rapidly with $L$ and $N$ and one is restricted to rather modest system sizes if one wishes to use exact diagonalization when calculating eigenstates and eigenvalues.

Hamiltonian (4.6) is furthermore translational invariant, and the dimension of the Hilbert space can therefore be reduced one step further. Defining the translation operator $\hat{T}$ as

$$
\hat{T}\left|n_{1}, n_{2}, \ldots, n_{L}\right\rangle=\left|n_{2}, \ldots, n_{L}, n_{1}\right\rangle,
$$

it is readily tested that both $\hat{H}$ and $\hat{N}$ commute with $\hat{T}$, and the Hamiltonian can therefore be further block diagonalized in a basis of mutual eigenstates to $\hat{T}$ and $\hat{N}$. We are thus looking for states which look the same, apart from a numerical factor, when translated one site. Translating this state through the whole lattice must also give back the same state, i.e. $\hat{T}^{L}=\hat{I}$, where $\hat{I}$ is the identity operator. This implies that the eigenvalues of $\hat{T}$, denoted $\tau_{k}$, obey $\tau_{k}^{L}=1 \Rightarrow \tau_{k}=e^{2 \pi k i / L}$, where $k=0, \pm 1, \ldots, \pm(L / 2-1),+L / 2$ ( $L$ even) or $k=0, \pm 1, \ldots, \pm(L-1) / 2(L$ odd). It is readily confirmed that states of the following type are eigenstates with eigenvalue $\tau_{k}$

$$
\left|\tau_{k}^{(\alpha)}\right\rangle=\sum_{j=0}^{L-1}\left(e^{-2 \pi k i / L} \hat{T}\right)^{j}\left|n_{1}^{(\alpha)}, \ldots, n_{L}^{(\alpha)}\right\rangle,
$$

where $\alpha$ distinguish different Fock states. These states will thus serve as a suitable basis to express Hamiltonian (4.6) in ${ }^{1}$. Note however that when generating a basis of states (4.11), one should not include two states such that

$$
\left\langle n_{1}^{(\alpha)}, \ldots, n_{L}^{(\alpha)}\left|\hat{T}^{s}\right| n_{1}^{(\beta)}, \ldots, n_{L}^{(\beta)}\right\rangle \neq 0,
$$

for any $s$, since these will result in the same state when plugged into (4.11), differing only by a phase factor. One should also note that Fock states which possess an additional translational symmetry, i.e.

$$
\hat{T}^{s}\left|n_{1}^{(\alpha)}, \ldots, n_{L}^{(\alpha)}\right\rangle=\left|n_{1}^{(\alpha)}, \ldots, n_{L}^{(\alpha)}\right\rangle
$$

for $s<L$, will generate the null-vector when plugged into (4.11) for certain $k$ values. A simple example is one particle in each lattice well of a two-site lattice, $|1,1\rangle$, which vanishes for $k=1$.

\footnotetext{
${ }^{1}$ These results are actually nothing more than the Bloch theorem in action [5]. The states defined by (4.11), and also linear combinations (with the same $k$ ) of them, have the structure of Bloch states, and $k$ is proportional to the crystal momentum.
} 


\subsection{BECs in Optical Lattices}

The derivation of the Bose-Hubbard Hamiltonian for a BEC in an optical lattice can be conducted in a quite straightforward manner by starting from a general bosonic many-body Hamiltonian of the form [63],

$$
\hat{H}=\int \hat{\Psi}^{\dagger}(\mathbf{r}) \hat{H}^{(1)}(\mathbf{r}) \hat{\Psi}(\mathbf{r}) \mathrm{d} \mathbf{r}+\frac{1}{2} \iint \hat{\Psi}^{\dagger}(\mathbf{r}) \hat{\Psi}^{\dagger}\left(\mathbf{r}^{\prime}\right) \hat{H}^{(2)}\left(\mathbf{r}, \mathbf{r}^{\prime}\right) \hat{\Psi}\left(\mathbf{r}^{\prime}\right) \hat{\Psi}(\mathbf{r}) \mathrm{d} \mathbf{r} \mathrm{d} \mathbf{r}^{\prime}
$$

where $\hat{H}^{(1)}$ is the part of the Hamiltonian that acts on one particle, i.e. kinetic energy and applied potentials, and $\hat{H}^{(2)}$ the part that acts on particle pairs, i.e. interaction energies. $\hat{\Psi}(\mathbf{r})$ and $\hat{\Psi}^{\dagger}(\mathbf{r})$ are the bosonic field operators that destroy and create a particle at position $\mathbf{r}$, respectively. These can be expanded in an arbitrary complete basis $\left\{f_{j}(\mathbf{r})\right\}$, so that

$$
\begin{array}{r}
\hat{\Psi}(\mathbf{r})=\sum_{j} f_{j}(\mathbf{r}) \hat{a}_{j} \\
\hat{\Psi}^{\dagger}(\mathbf{r})=\sum_{j} f_{j}^{*}(\mathbf{r}) \hat{a}_{j}^{\dagger},
\end{array}
$$

where $\hat{a}_{j}$ and $\hat{a}_{j}^{\dagger}$ are bosonic annihilation and creation operators, respectively, which destroy and create a particle in a state described by the wave function $f_{j}(\mathbf{r})^{2}$.

Hamiltonian (4.14) is generally too complicated to work with directly, and it is thus necessary to make suitable approximations which capture the system's main physical features. In the case of the Bose-Hubbard model, these approximations originate from that we are considering a cold, weakly interacting, dilute boson gas in a deep optical lattice ${ }^{3}$. We will for generality make the derivation for a three-dimensional lattice, but it can equally well be done for both one and two dimensions. Remember from Sec. 2.1.2 that it was possible to reduce the dimensionality by increasing the trapping or lattice potential in certain directions. We will return to this topic at the end of this section.

For a deep optical lattice, it seems reasonable that the field operators should be expanded in states which are localized around the lattice sites (compare with the analogous expression of the macroscopic wave function in (3.18)). These are readily available in the form of Wannier functions $w_{n, \mathbf{R}}(\mathbf{r})$, defined as Fourier components to the Bloch functions $\psi_{n, \mathbf{q}}(\mathbf{r})$, which should be familiar from solid state physics as the eigenstates of a single electron moving in a perfect crystal [5]. Note though that this situation is equivalent to the noninteracting Hamiltonian (4.14) with a periodic potential, which then also will have Bloch functions as eigenstates. These have the general form

$$
\psi_{n, \mathbf{q}}(\mathbf{r})=e^{i \mathbf{q} \cdot \mathbf{r}} u_{n, \mathbf{q}}(\mathbf{r})
$$

\footnotetext{
${ }^{2}$ The field operator $\hat{\Psi}\left(\hat{r}_{j}\right)$ can be viewed as an annihilation operator connected to the eigenstate of the position operator with eigenvalue $\mathbf{r}_{j}$, i.e. $\delta\left(\mathbf{r}-\mathbf{r}_{j}\right)$. The Kronecker delta in (4.2) will then be exchanged for a Dirac delta, since $\mathbf{r}$ is a continuous set of eigenvalues.

${ }^{3}$ It is actually the validity of the approximations which defines which regimes we call cold, dilute, etc.
} 
where $\mathbf{q}$ is the quasimomentum, $n$ the band index and $u_{n, \mathbf{q}}(\mathbf{r})$ a function with the same periodicity as the lattice. The relationship between Wannier and Bloch functions is thus given by

$$
\begin{array}{r}
\psi_{n, \mathbf{q}}(\mathbf{r})=\sum_{\mathbf{R}} w_{n, \mathbf{R}}(\mathbf{r}) e^{i \mathbf{q} \cdot \mathbf{R}}, \\
w_{n, \mathbf{R}}(\mathbf{r})=\frac{1}{V_{B Z}} \int \psi_{n, \mathbf{q}}(\mathbf{r}) e^{-i \mathbf{q} \cdot \mathbf{R}} \mathrm{d} \mathbf{q},
\end{array}
$$

where $\mathbf{R}$ denotes the lattice vectors and the integration runs over the first Brillouin zone, which has volume $V_{B Z}$. The Wannier functions will, just as the Bloch functions, form a complete orthonormal basis, when properly normalized. It can be readily verified, for instance by utilizing that $\psi_{n, \mathbf{q}}(\mathbf{r}+\mathbf{R})=e^{i \mathbf{q} \cdot \mathbf{R}} \psi_{n, \mathbf{q}}(\mathbf{r})$, that they must have the form $w_{n, \mathbf{R}}(\mathbf{r})=w_{n}(\mathbf{r}-\mathbf{R})$, i.e. that all Wannier functions in a band are copies of each other, only translated by a lattice vector.

The Wannier functions are exponentially decaying for lattices with simple bands, but with a decay rate that depends on the depth of the lattice potential [169]. They can therefore be rather wide for a shallow lattice, but should become more localized around a single lattice site for increasing depth.

For a separable periodic potential $\hat{V}_{p e r}(\mathbf{r})=\sum_{j=1}^{3} \hat{V}_{j}\left(x_{j}\right)$, which is e.g. the common case of an optical lattice generated by a set of perpendicular laser beams, the problem can effectively be reduced to one dimension, for which Wannier functions have been studied in detail by Kohn [170]. The form of the Wannier functions depends on the global phase of the Bloch functions ${ }^{4}$, and there is one and only one choice of the phases which will make $\left.w_{n}(x) i\right)$ real, $\left.i i\right)$ even or odd, iii) exponentially decaying.

Because of the low temperature, one can assume that only the lowest Wannier band is occupied ${ }^{5}$. This also requires that the interaction energies, which are discussed later, should be smaller than the band gap, thus invoking the requirement of weak interactions. Band index will therefore be omitted hereafter.

For a deep sinusoidal lattice, the lattice wells can (locally) be approximated with harmonic oscillators, and the Wannier functions can thus be replaced with the corresponding eigenstates [76]. One should however note that no matter how deep the lattice gets, the Wannier function will never converge completely to the harmonic oscillator state, which is decaying Gaussian rather than exponential, but there will be a large overlap between the two states. This is useful for obtaining analytical expressions [76].

Assume now that the $\hat{H}^{(1)}$-term of Hamiltonian (4.14), apart from the kinetic energy, contains contributions from a periodic potential $\hat{V}_{\text {per }}(\mathbf{r})$, due to the optical lattice, and possibly also from a slowly varying trapping potential $\hat{V}_{\text {trap }}(\mathbf{r})$. The trapping potential is taken to be essentially constant over a couple of lattice sites, so that $\int w\left(\mathbf{r}-\mathbf{R}^{\prime}\right) \hat{V}_{\text {trap }}(\mathbf{r}) w(\mathbf{r}-\mathbf{R}) \mathrm{d} \mathbf{r}=V_{\text {trap }}(\mathbf{R}) \delta_{\mathbf{R}, \mathbf{R}^{\prime}}$. Replacing the field operators in this term with the corresponding Wannier functions, will then lead

\footnotetext{
${ }^{4}$ This is not only the case in one dimension, but is a general property, as can be seen from Eq. (4.17b).

${ }^{5}$ It is also possible to create BECs in higher bands [171-173].
} 
to

$$
\hat{H}=\sum_{\mathbf{R} \neq \mathbf{R}^{\prime}} \Upsilon\left(\mathbf{R}^{\prime}-\mathbf{R}\right) \hat{a}_{\mathbf{R}^{\prime}}^{\dagger} \hat{a}_{\mathbf{R}}+\sum_{\mathbf{R}} \epsilon_{\mathbf{R}} \hat{n}_{\mathbf{R}}
$$

where

$$
\begin{aligned}
\Upsilon\left(\mathbf{R}^{\prime}-\mathbf{R}\right) & =\int w\left(\mathbf{r}-\mathbf{R}^{\prime}\right)\left(\frac{\hat{p}^{2}}{2 m}+\hat{V}_{\text {per }}(\mathbf{r})\right) w(\mathbf{r}-\mathbf{R}) \mathrm{d} \mathbf{r}, \\
\epsilon_{\mathbf{R}} & =\Upsilon(\mathbf{0})+V_{\text {trap }}(\mathbf{R}) .
\end{aligned}
$$

$\Upsilon\left(\mathbf{R}^{\prime}-\mathbf{R}\right)$ is the matrix element for the hopping of a boson between sites $\mathbf{R}$ and $\mathbf{R}^{\prime}$. These are, just as the Wannier functions, decreasing with distance, so that only the nearest neighbor hopping is non-negligible for sufficiently deep lattices. It is thus only the nearest neighbor hopping that will be of interest and the corresponding matrix element will generally be negative ${ }^{6}[76]$, and we will from now on therefore denote this $\Upsilon$ with $-J$, and take $J \geq 0$. Estimations on how the ratio between matrix elements for different hopping lengths depend on the lattice depth can be obtained by replacing the Wannier functions in (4.19a) with harmonic oscillator states [76]. The constant $\epsilon_{\mathbf{R}}$ on the other hand gives the single particle on-site energy at site $\mathbf{R}$. The first integral in Hamiltonian (4.14) can thus be approximated with

$$
-J \sum_{<\mathbf{R}, \mathbf{R}^{\prime}>} \hat{a}_{\mathbf{R}}^{\dagger} \hat{a}_{\mathbf{R}^{\prime}}+\sum_{\mathbf{R}} \epsilon_{\mathbf{R}} \hat{n}_{\mathbf{R}} .
$$

Let us now shift focus to the second integral in (4.14) and interactions. For a weakly interacting, dilute gas, collisions are rare events and can therefore be taken to always be between only two particles at a time. Because of the low temperatures, the collisions are also assumed to be entirely of the $s$-wave scattering type, since higher angular momentum scattering will be frozen out by the centrifugal barrier. The interaction potential can then be approximated with a contact pseudopotential of the form [9]

$$
V_{\text {int }}(\mathbf{r})=\frac{4 \pi \hbar^{2} a_{s}}{M_{r}} \delta(\mathbf{r})
$$

where $M_{r}$ is the reduced mass, and $a_{s}$ is the $s$-wave scattering length. This will be the relevant parameter that characterizes the interaction strength, larger $a_{s}$ meaning stronger interactions, with positive (negative) value indicating repulsion (attraction).

Inserting $\hat{H}^{(2)}$ with (4.21) in the second integral in (4.14) leads to

$$
\hat{H}_{\text {int }}=\frac{1}{2} \frac{4 \pi \hbar^{2} a_{s}}{M_{r}} \int \hat{\Psi}^{\dagger}(\mathbf{r}) \hat{\Psi}^{\dagger}(\mathbf{r}) \hat{\Psi}(\mathbf{r}) \hat{\Psi}(\mathbf{r}) \mathrm{d} \mathbf{r} .
$$

Expanding the field operators, once again, in deep potential Wannier functions, one realizes that (4.22) is dominated by the terms $\sim \hat{a}_{\mathbf{R}}^{\dagger} \hat{a}_{\mathbf{R}}^{\dagger} \hat{a}_{\mathbf{R}} \hat{a}_{\mathbf{R}}=\hat{n}_{\mathbf{R}}\left(\hat{n}_{\mathbf{R}}-1\right)$, and that the interaction part of (4.14) therefore can be approximated with

$$
\frac{U}{2} \sum_{\mathbf{R}} \hat{n}_{\mathbf{R}}\left(\hat{n}_{\mathbf{R}}-1\right)
$$

\footnotetext{
${ }^{6}$ Bose-Hubbard models with positive hopping matrix element have been studied, e.g. in Ref. [174] where it is generated by a fast, time-periodic acceleration of the lattice.
} 
where

$$
U=\frac{4 \pi \hbar^{2} a_{s}}{M_{r}} \int|w(\mathbf{r})|^{4} \mathrm{~d} \mathbf{r} .
$$

Putting together (4.20) and (4.23) finally gives us the Bose-Hubbard model

$$
\hat{H}=-J \sum_{<\mathbf{R}, \mathbf{R}^{\prime}>} \hat{a}_{\mathbf{R}}^{\dagger} \hat{a}_{\mathbf{R}^{\prime}}+\frac{U}{2} \sum_{\mathbf{R}} \hat{n}_{\mathbf{R}}\left(\hat{n}_{\mathbf{R}}-1\right)+\sum_{\mathbf{R}} \epsilon_{\mathbf{R}} \hat{n}_{\mathbf{R}} .
$$

If the site-dependence of the on-site potential can be neglected so that $\epsilon_{\mathbf{R}}=\epsilon$, then the Hamilonian can be rescaled with the (constant) total number of particles, $\hat{H}-\epsilon \hat{N}=\hat{H}-\epsilon N$, which gives the same Hamiltonian as (4.1)

The derivation of the Bose-Hubbard model in lower dimensions can be done in an almost identical manner. The difference is that one has to handle the confinement in the directions perpendicular to the lattice. The potential in these directions is usually taken to be approximately harmonic, and it is assumed that the frequency $\omega_{\perp}$, and thereby the energy separation $\hbar \omega_{\perp}$ between eigenstates, is sufficiently large (compared to interaction and thermal energies) that only the lowest eigenstate has to be included [175]. This is analogous to why only the lowest Wannier band was considered.

For a one-dimensional lattice (pointing in $z$-direction), this means that the state localized on site $\mathbf{R}=\left(x_{0}, y_{0}, z_{0}\right)$ will be given by $h_{0}\left(x-x_{0}\right) h_{0}\left(y-y_{0}\right) w\left(z-z_{0}\right)$ instead of $w(\mathbf{r}-\mathbf{R})$, where $h_{0}(x)$ is the harmonic oscillator ground state. Using this state will, if the on-site potentials' site dependence is neglected, produce Hamiltonian (4.6).

\subsection{Extended Bose-Hubbard Models}

Similar to the DNLS model (Sec. 3.4), there are regimes where the validity of the assumptions and approximations behind the Bose-Hubbard model may come into question, and it is necessary to expand the model [176]. One of these assumptions regards the decay rate of the Wannier functions, and was used to motivate which terms of the Hamiltonian, when expanded in these functions, that should be included. Approximating the Wannier functions with harmonic oscillator ground states, Mazzarella et al $[177]^{7}$ showed that the lowest order corrections to this assumption come from the interaction part of the Hamiltonian. Including also these terms, for a one-dimensional homogeneous $\left(\epsilon_{j}=\epsilon\right)$ lattice, leads to the following Hamiltonian

$$
\begin{array}{r}
\hat{H}=\sum_{j=1}^{L}\left(Q_{1} \hat{n}_{j}+Q_{2}\left(\hat{a}_{j}^{\dagger} \hat{a}_{j+1}+\hat{a}_{j+1}^{\dagger} \hat{a}_{j}\right)+Q_{3} \hat{n}_{j}^{2}\right. \\
+Q_{4}\left[4 \hat{n}_{j} \hat{n}_{j+1}+\left(\hat{a}_{j+1}^{\dagger}\right)^{2}\left(\hat{a}_{j}\right)^{2}+\left(\hat{a}_{j}^{\dagger}\right)^{2}\left(\hat{a}_{j+1}\right)^{2}\right] \\
\left.+2 Q_{5}\left[\hat{a}_{j}^{\dagger}\left(\hat{n}_{j}+\hat{n}_{j+1}\right) \hat{a}_{j+1}+\hat{a}_{j+1}^{\dagger}\left(\hat{n}_{j+1}+\hat{n}_{j}\right) \hat{a}_{j}\right]\right),
\end{array}
$$

\footnotetext{
${ }^{7}$ This paper contains a misprint so that it appears that they are not studying the same model as we are in paper I and paper III, which they however do.
} 
where the parameter notation has been changed from (4.6) to agree with paper I and III. The first three terms are essentially the ordinary Bose-Hubbard model, with $Q_{1}=-U / 2+\epsilon, Q_{2}=-J$ and $Q_{3}=U / 2$, while the last two terms are the extension. Just as $\hat{a}_{j+1}^{\dagger} \hat{a}_{j}$ indicates nearest neighbor tunneling and $\left(\hat{n}_{j}-1\right) \hat{n}_{j}$ is related to the on-site energy, these new terms can be associated with simple interaction or tunneling processes:

- $\hat{n}_{j} \hat{n}_{j+1}$ is related to the interaction energy between atoms at neighboring sites.

- $\left(\hat{a}_{j+1}^{\dagger}\right)^{2}\left(\hat{a}_{j}\right)^{2}$ is coherent tunneling of two particles.

- $\hat{a}_{j+1}^{\dagger} \hat{n}_{j+1} \hat{a}_{j}$ and $\hat{a}_{j+1}^{\dagger} \hat{n}_{j} \hat{a}_{j}$ are density dependent tunneling, since they depend on the number of particles at the site the particle tunnels to and from, respectively. This can also be called conditioned tunneling, since $\hat{a}_{j+1}^{\dagger} \hat{n}_{j+1} \hat{a}_{j}$ vanishes when the site the particle tunnels to is empty, and $\hat{a}_{j+1}^{\dagger} \hat{n}_{j} \hat{a}_{j}$ vanishes when the site the particle tunnels from would become empty.

This model has been used in several theoretical [177-182] as well as some experimental work [183].

An analogous extended model can also be produced when effects of higher bands are taken into account, in a "dressed" lowest band model [184, 185].

A similar model, with the same terms as (4.26) but with separate parameters for the neighbor interaction term and two-particle tunneling, was used for a BEC with dipolar interactions [186], i.e. with longer range interactions which are not well described by the contact potential. It is also common in this context to use a Bose-Hubbard model extended only with the nearest neighbor interaction term [187-190].

Density dependent tunneling (i.e. the term proportional to $Q_{5}$ in Eq. (4.26)) has been experimentally observed with a BEC in a tilted optical lattice [191]. It has also been observed by using a rapid periodic modulation of the interaction strength (using Feshbach resonance), which effectively gives rise to a density dependent coupling parameter $J$ in (4.1) [192]. Note that this is the experimental scheme discussed in Refs. [35,36], however within a DNLS context, for realizing lattice compactons (see Sec. 1.3.4).

Bose-Hubbard models can also be used for BECs in higher bands [193]. This introduces new degrees of freedom for the bosons, e.g. that the orbitals can point in different directions, which leads to anisotropic tunneling and interactions [194].

\subsection{Coherent States}

Coherent states play an important role when studying the correspondence between classical and quantum physics (in our case between Bose-Hubbard and DNLS models), and are generally considered to be the most 'classical-like' quantum states. The concept of a coherent state was first introduced by Schrödinger already in 
$1926^{8}[195]$ as a minimum uncertainty state of the harmonic oscillator. This is a property which is desirable for classical-like states, as the Heisenberg uncertainty principle is one of the most distinct differences between classical and quantum physics. Schrödinger's work received fairly little attention at the time (most physicists were probably busy with developing quantum theory itself), and the broader interest in coherent states was instead sparked by the work by Roy Glauber (and others [196]) within quantum optics in the 1960's [197]. He used these states to quantum mechanically describe (i.e. in terms of photons) coherent classical light, hence the name ${ }^{9}$. To distinguish these particular coherent states from other generalized versions, which will be discussed below, we will refer to them as Glauber coherent states (GCS). Glauber defined the (single mode) coherent states in three different ways [197]:

(i) Eigenstates to the annihilation operator of the harmonic oscillator:

$$
\hat{a}|\alpha\rangle_{(G C S)}=\alpha|\alpha\rangle_{(G C S)},
$$

where $\alpha$ is a complex number. From this definition, it directly follows that

$$
\langle\hat{n}\rangle=|\alpha|^{2} \text {. }
$$

One may from this definition also construct the Glauber coherent states in terms of eigenstates of the one-dimensional harmonic oscillator, which we denote as $|n\rangle$, with the associated energy $E_{n}=(n+1 / 2) \hbar \omega$. Using that $\hat{a}|n\rangle=\sqrt{n}|n-1\rangle$, one can verify that the following normalized state

$$
|\alpha\rangle_{(G C S)}=e^{-|\alpha|^{2} / 2} \sum_{n} \frac{\alpha^{n}}{\sqrt{n !}}|n\rangle,
$$

satisfies Eq. (4.27). The time evolution of state (4.29) in the harmonic oscillator potential with frequency $\omega$ is easily calculated to be

$$
|\alpha, t\rangle_{(G C S)}=e^{-|\alpha|^{2} / 2} \sum_{n} \frac{\alpha^{n}}{\sqrt{n !}} e^{-i \omega(n+1 / 2) t}|n\rangle=e^{-i \omega t / 2}\left|\alpha e^{-i \omega t}\right\rangle_{(G C S)},
$$

meaning that it remains a Glauber coherent state, only phase twisted.

(ii) Minimum uncertainty states. The position and momentum operator can be expressed with the harmonic oscillator creation and annihilation operator as

$$
\begin{gathered}
\hat{q}=\sqrt{\frac{2 \hbar}{m \omega}} \frac{\left(\hat{a}+\hat{a}^{\dagger}\right)}{2}, \\
\hat{p}=\sqrt{2 \hbar m \omega} \frac{\left(\hat{a}-\hat{a}^{\dagger}\right)}{2 i} .
\end{gathered}
$$

Glauber coherent states fulfill the Heisenberg uncertainty principle with equality, i.e.

$$
\Delta q \cdot \Delta p=\frac{\hbar}{2}
$$

\footnotetext{
${ }^{8}$ This is the same year he published the paper which introduces his eponymous wave equation.

${ }^{9}$ Glauber received the 2005 Nobel Prize in Physics "for his contribution to the quantum theory of optical coherence".
} 
where

$$
(\Delta x)^{2}:=\left\langle\alpha\left|\hat{x}^{2}\right| \alpha\right\rangle_{(G C S)}-\langle\alpha|\hat{x}| \alpha\rangle_{(G C S)}^{2} .
$$

Introducing the dimensionless quadrature operators,

$$
\begin{aligned}
& \hat{X}_{1}=\frac{\left(\hat{a}+\hat{a}^{\dagger}\right)}{2}, \\
& \hat{X}_{2}=\frac{\left(\hat{a}-\hat{a}^{\dagger}\right)}{2 i},
\end{aligned}
$$

Glauber coherent states are furthermore defined to have equal uncertainty in both quadratures, i.e. $\left(\Delta X_{1}\right)^{2}=\left(\Delta X_{2}\right)^{2}=1 / 4$. One can verify that state (4.27) fulfills these conditions. The quadrature operators also have the property that

$$
\begin{aligned}
& \left\langle\alpha\left|\hat{X}_{1}\right| \alpha\right\rangle_{(G C S)}=\operatorname{Re}(\alpha), \\
& \left\langle\alpha\left|\hat{X}_{2}\right| \alpha\right\rangle_{(G C S)}=\operatorname{Im}(\alpha) .
\end{aligned}
$$

Glauber coherent states can due to this be represented graphically as a circle in the complex plane (with the quadrature on either axis) which is centered at $\alpha$ and with diameter $\Delta X_{1}=\Delta X_{2}=1 / 2$, representing the uncertainty of the state. The time evolution of the coherent state (4.30) means that the circle rotates in the complex place, with angular frequency $\omega$, in clear correspondence with the time evolution of the classical harmonic oscillator (the projection onto the $X_{2}$-axis is related to the classical electric field of a plane wave, which Glauber coherent states can be used to describe in quantum optics).

There are other states which fulfill (4.32) but have $\left(\Delta X_{1}\right)^{2} \neq\left(\Delta X_{2}\right)^{2}$, so that the uncertainty of one quadrature is reduced at the expense of the other. These are called squeezed states, and are instead represented as ellipses in the complex plane [64].

(iii) The third definition states that Glauber coherent states can be constructed from the vacuum state through

$$
|\alpha\rangle_{(G C S)}=D(\alpha)|0\rangle,
$$

where $D(\alpha)$ is a displacement operator

$$
D(\alpha)=\exp \left(\alpha \hat{a}^{\dagger}-\alpha^{*} \hat{a}\right)
$$

By using the Baker-Campbell-Hausdorff formula,

$$
\exp \left(\alpha \hat{a}^{\dagger}-\beta \hat{a}\right)=\exp (\alpha \beta / 2) \exp (-\beta \hat{a}) \exp \left(\alpha \hat{a}^{\dagger}\right)
$$

Eq. (4.36) can be recast in the form of Eq. (4.27).

\section{Generalized Coherent States}

The third definition of Glauber coherent states is arguably the most abstract of the three, but it has served as the basis for a generalization of coherent states to systems which are not connected with the harmonic oscillator. Mainly through 
the work of A. Perelomov and R. Gilmore, a theory of generalized coherent states has been developed that is based around Lie groups [196]. Without going into much detail (see Ref. [196]), this theory gives an algorithm for constructing coherent states for the quantum mechanical system of interest, through a generalized displacement operator acting on a reference state (cf. Eq. (4.36)). The generalized coherent states that we are primarily interested in are called $\mathrm{SU}(\mathcal{L})$ coherent states, $\mathcal{L}$ being the number of sites ( $L$ is reserved for the number of ring-sites), and are given by

$$
\begin{array}{r}
|\mathrm{SU}(\mathcal{L})\rangle=\frac{1}{\sqrt{N^{N} N !}}\left(\sum_{\mathbf{R}} \psi_{\mathbf{R}} a_{\mathbf{R}}^{\dagger}\right)^{N}|0,0, \ldots, 0\rangle \\
\sum_{\mathbf{R}}\left|\psi_{\mathbf{R}}\right|^{2}=N .
\end{array}
$$

It can be shown that the operators in Eq. (4.39a) belong to the $\mathrm{SU}(\mathcal{L})$-group, hence the name ${ }^{10}[175]$. An important property of coherent states is that there is a one-to-one correspondence between them and the states of classical phase space, and it is evident that states (4.39) indeed are in one-to-one correspondence with the state vectors of the DNLS model (3.1), as both are described by the $\mathcal{L}$-dimensional complex vectors $\left\{\psi_{\mathbf{R}}\right\}$ with a certain norm (3.5).

The $\operatorname{SU}(\mathcal{L})$ coherent state is in some work called the Hartree wave function [198], since it is obtained by taking $N-1$ tensor products of the same single particle state

$$
\left(\sum_{\mathbf{R}} \psi_{\mathbf{R}} a_{\mathbf{R}}^{\dagger}\right)|0,0, \ldots, 0\rangle
$$

\subsection{Connection to the DNLS Model}

The DNLS model can be derived from the Bose-Hubbard model by using the so called time-dependent variational principle (TDVP), which is an extension of the familiar time-independent Rayleigh-Ritz variational method and a quite general method for producing approximate macroscopic wave functions for manybody systems [199]. The basic idea is to describe the system with a 'good' state, $|\tilde{\Phi}\rangle$, which contains some variational parameters that are determined by demanding that it should fulfill the time-dependent Schrödinger equation on average, $\langle\tilde{\Phi}|i \hbar \partial / \partial t-\hat{H}| \tilde{\Phi}\rangle=0$. Putting $|\tilde{\Phi}\rangle=e^{i \mathcal{S} / \hbar}|\Phi\rangle$ leads to

$$
\dot{\mathcal{S}}=i \hbar\langle\Phi|\partial / \partial t| \Phi\rangle-\langle\Phi|\hat{H}| \Phi\rangle
$$

where $|\Phi\rangle$ is the trial macroscopic state. It should be chosen to contain as much information as possible on the microscopic dynamics, and one should also be able

\footnotetext{
${ }^{10}$ Coherent states are sometimes named after the group which the quantum Hamiltonian is associated with, which is in contrast to our convention where $\mathrm{SU}(\mathcal{L})$ refers to the group of the generalized displacement operator. Note especially that the $\mathrm{SU}(2)$ coherent state discussed in Ref. [196] deals with spin systems, and is not the two-site version of (4.39).
} 
to associate it with a set of parameters which describe the most important physical processes of the system. These parameters will then become the dynamical variables of the semi-classical model. One can then associate $\dot{\mathcal{S}}$ and $\langle\Phi|\hat{H}| \Phi\rangle$ with an effective Langrangian and Hamiltonian, respectively, and with the help of Hamilton's equations of motion determine the time-evolution of the semi-classical system [199].

Amico and Penna [199] used this procedure with a tensor product of Glauber coherent states (4.29) as the macroscopic trial state, where $n$ in Eq. (4.29) now is the number of bosons at a site. These are local states, i.e. they are describing only a single site, which is why it is necessary to take a tensor product to describe the full lattice. Plugging in $|\Phi\rangle=\bigotimes_{\mathbf{R}}\left|\psi_{\mathbf{R}}\right\rangle_{(G C S)}$ to $H=\langle\Phi|\hat{H}| \Phi\rangle$, with $\hat{H}$ being Hamiltonian (4.1), gives the following semi-classical Hamiltonian,

$$
H=-J \sum_{<\mathbf{R}, \mathbf{R}^{\prime}>} \psi_{\mathbf{R}}^{*} \psi_{\mathbf{R}^{\prime}}+\frac{U}{2} \sum_{\mathbf{R}}\left|\psi_{\mathbf{R}}\right|^{2}\left(\left|\psi_{\mathbf{R}}\right|^{2}-1\right),
$$

where $\psi_{\mathbf{R}}$ and $i \hbar \psi_{\mathbf{R}}^{*}$ are the canonical variables, generating the following equations of motion,

$$
i \hbar \frac{d \psi_{\mathbf{R}}}{d t}=-J \sum_{<\mathbf{R}^{\prime}>} \psi_{\mathbf{R}^{\prime}}+U\left(\psi_{\mathbf{R}}\left|\psi_{\mathbf{R}}\right|^{2}-\frac{\psi_{\mathbf{R}}}{2}\right),
$$

where the summation over $\mathbf{R}^{\prime}$ runs over all the nearest neighbors of $\mathbf{R}$. As in Sec. 3.2, the term $\sim \psi_{\mathbf{R}}$ in (4.43) can be removed by the transformation $\psi_{\mathbf{R}} \mapsto$ $\exp (i U / 2 \hbar) \psi_{\mathbf{R}}$, which corresponds to making a replacement of the Hamiltonian according to $H \mapsto H-U \mathcal{N} / 2$, which is just a shift of the energy scale since the norm $\mathcal{N}$ (Eq. (3.5)) is conserved. These two expressions have the form of the DNLS equation (3.1) and Hamiltonian (3.3), with $J=\delta, U=-\gamma$, and $t / \hbar \rightarrow t$.

A different approach to derive the DNLS model is to consider the time-evolution of the annihilation operator, given by Heisenberg's equation of motion,

$$
i \hbar \frac{d \hat{a}_{\mathbf{R}}}{d t}=\left[\hat{a}_{\mathbf{R}}, \hat{H}\right]=-J \sum_{<\mathbf{R}^{\prime}>} \hat{a}_{\mathbf{R}^{\prime}}+U \hat{a}_{\mathbf{R}}^{\dagger} \hat{a}_{\mathbf{R}} \hat{a}_{\mathbf{R}},
$$

where $\hat{H}$ is the Bose-Hubbard Hamiltonian (4.1). Taking the expectation value of this equation with a tensor product of Glauber coherent states leads to essentially the same equation as (4.43), differing only by the insignificant term $\sim \psi_{\mathbf{R}}$ [200]. It is actually quite common in the literature to just make the substitution $\psi_{\mathbf{R}} \leftrightarrow \hat{a}_{\mathbf{R}}$ when 'studying the quantum mechanical version of...' or vice versa.

The drawback of the Glauber coherent states is that they do not have a specified number of particles; in contrast to a Bose-Hubbard eigenstate these are only conserved on average. One can instead use $\mathrm{SU}(\mathcal{L})$-coherent states $(4.39)[175,198]$, which do conserve the number of particles $N$. The $\mathrm{SU}(\mathcal{L})$-coherent states will produce an almost identical DNLS model as when using Glauber coherent states, the difference is a numerical factor $(N-1) / N$ attached to the nonlinear term. The two models are thus equivalent for large $N$.

With the same techniques as above it is also possible to derive the extended DNLS model (3.26) from the extended Bose-Hubbard model (4.26). Using Glauber 
coherent states on (4.26) will lead to [201]

$$
\begin{array}{r}
H=\sum_{j}\left(\left(Q_{1}+Q_{3}\right)\left|\psi_{j}\right|^{2}+Q_{2}\left(\psi_{j} \psi_{j+1}^{*}+\psi_{j+1} \psi_{j}^{*}\right)\right. \\
+Q_{3}\left|\psi_{j}\right|^{4}+Q_{4}\left(4\left|\psi_{j}\right|^{2}\left|\psi_{j+1}\right|^{2}+\psi_{j}^{2} \psi_{j+1}^{* 2}+\psi_{j+1}^{2} \psi_{j}^{* 2}\right) \\
\left.+2 Q_{5}\left[\psi_{j} \psi_{j+1}\left(\psi_{j}^{* 2}+\psi_{j+1}^{* 2}\right)+\left(\psi_{j}^{2}+\psi_{j+1}^{2}\right)\left(\psi_{j}^{*} \psi_{j+1}^{*}\right)\right]\right),
\end{array}
$$

with $\sum_{j}\left|\psi_{j}\right|^{2}=N$, which is the same as Eq. (3.29) with the proper parameter identifications. Using SU $(L)$-coherent states instead leads to the same Hamiltonian apart from that the last two rows will be multiplied by the factor $(N-1) / N$. Equation (4.45) inserted in Hamilton's equations of motion leads to

$$
\begin{array}{r}
i \hbar \frac{d \psi_{j}}{d t}=\left(Q_{1}+Q_{3}\right) \psi_{j}+Q_{2}\left(\psi_{j-1}+\psi_{j+1}\right)+2 Q_{3} \psi_{j}\left|\psi_{j}\right|^{2} \\
+Q_{4}\left[4 \psi_{j}\left(\left|\psi_{j+1}\right|^{2}+\left|\psi_{j-1}\right|^{2}\right)+2 \psi_{j}^{*}\left(\psi_{j+1}^{2}+\psi_{j-1}^{2}\right)\right] \\
+2 Q_{5}\left[2\left|\psi_{j}\right|^{2}\left(\psi_{j+1}+\psi_{j-1}\right)+\left(\psi_{j}^{2}+\psi_{j+1}^{2}\right) \psi_{j+1}^{*}+\left(\psi_{j}^{2}+\psi_{j-1}^{2}\right) \psi_{j-1}^{*}\right],
\end{array}
$$

which is equivalent to $(3.26)$.

Looking at the Hamiltonians of both the ordinary and the extended DNLS model, one can see how the parameters should scale to have a well-defined classical limit $N \rightarrow \infty$, i.e. so that the Hamiltonian has a finite value. Since $\left|\psi_{\mathbf{R}}\right|$ at most can be of order $\sqrt{N}$, we can conclude that $Q_{1}, Q_{2}$ and $J$ should scale as $N^{-1}$ while $Q_{3}, Q_{4}, Q_{5}$ and $U$ scale as $N^{-2}$. This also indicates how Bose-Hubbard models with different numbers of particles can be compared, i.e. in which parameter regimes one should look for similar behavior [201].

\subsection{Quantum Discrete Breathers}

When one tries to transfer the concept of a DB to the quantum world, one realizes that in a translationally invariant system, all eigenstates must obey the Bloch theorem, meaning that they necessarily are delocalized. So how can localization possibly arise in quantum mechanics? We need to be a bit more specific with what we mean by delocalization here. Consider the general form of an eigenstate with energy $E_{k}$,

$$
\left|E_{k}\right\rangle=\sum_{\alpha} c_{\alpha}\left|\tau_{k}^{(\alpha)}\right\rangle
$$

where $\left|\tau_{k}^{(\alpha)}\right\rangle$ is a basis state of type (4.11). Now, state (4.47) is not delocalized in the sense that upon measurement there will necessarily be an equal amount of particles on each site, but rather that the probability of finding a particular number of particles is the same for every site. The eigenstate may therefore actually have a high probability of having many particles located on a few sites, but there cannot be any sites that are more likely than others to be occupied.

But a classical breather is of course localized on specific sites, and to create a quantum state which is truly localized, one needs to take a superposition of 
eigenstates. The timescale for delocalization of such a state is then determined by the energy differences between the eigenstates in this superposition - smaller energy differences meaning longer delocalization times. The expectation is therefore that a classical discrete breather corresponds in the quantum world to a superposition of quasi-degenerate eigenstates [202]. Using perturbation theory, Bernstein et al [165] derived formulas for the energy splitting between the relevant eigenstates of Hamiltonian (4.6) in the strong (attractive) interaction limit,

$$
\Delta E=\frac{(\Delta \lambda) N J^{N}}{(N-1) !(-U)^{N-1}}+\mathcal{O}\left(\frac{J^{N+1}}{(-U)^{N}}\right)
$$

where $\Delta \lambda$ is a constant, related to the inter-site coupling, for which

$$
\Delta \lambda \leq 2(L-1)
$$

The eigenstates in this superposition need of course to be localized themselves in the 'eigenstate-sense' mentioned above. One may also argue that the most important question is not whether the quantum state is localized on a specific site, but rather if a measurement will result in a localized configuration with high probability, and that one indeed can focus on separate eigenstates [203]. In either case, there are different quantities that can be used to characterize how well localized an eigenstate is. One example is certain correlation functions [204], e.g. $\left\langle\hat{n}_{\mathbf{R}} \hat{n}_{\mathbf{R}+\mathbf{d}}\right\rangle$ which should decrease with increasing $|\mathbf{d}|$. Another one is $\left\langle\left(\hat{a}_{\mathbf{R}}^{\dagger}\right)^{M}\left(\hat{a}_{\mathbf{R}}\right)^{M}\right\rangle, M$ being a positive integer, which for a delocalized eigenstate should drop as $M$ becomes large while it will remain finite for a state with a significant probability of finding many of the system's particles on one site [175, 205].

The dimensionality of the quantum lattice problem, given by Eq. (4.9), is quite rapidly growing with the number of sites and particles, which has restricted the systems that are computationally accessible to quite modest sizes, at least if one wishes to use exact diagonalization of the Hamiltonian. This is probably a reason why there has been much less work done on quantum discrete breathers as compared to the classical ones [206].

The work done has typically been with either many particles on few lattice sites or vice versa. It is generally not sensible to talk about localization in a small lattice, but one can however study related concepts. In Sec. 3.7 we mentioned that one-dimensional DNLS models undergo a self-trapping transition, where below a critical value of the inter-site coupling $\delta$ (for attractive interactions), the uniform state $\psi_{n}=\sqrt{\mathcal{N} / L}$ becomes unstable and a symmetry-broken ground state emerges [144].

The self-trapping transition occurs already for the dimer, and quantum signatures of it have been studied quite extensively due to the system's simplicity. One signature of the classical transition can be seen in the quantum energy spectrum, where the energy splitting between the two lowest eigenstates decreases considerably close to the transition $[166,198,207]$. Signatures can also be seen in the ground state's probability distribution over different Fock states $|N-n, n\rangle$. When this distribution is plotted as a function of $n$, it shows a peak at (and is symmetric 
around) the state $|N / 2, N / 2\rangle$ in the non-self-trapped regime. The classical transition is signaled by a significant decrease in the population of Fock states with $n \approx N / 2$, while instead two peaks appear around states with $n=N / 2 \pm d$, where $d$ increases as one moves further into the self-trapped regime $[207,208]$. For large $d$, this type of state is sometimes called a Schrödinger-cat state, since it is a superposition of two macroscopically distinguishable states, corresponding to the classical self-trapped states with higher amplitude on either site. A general effect of finite $N$ is that the quantum transition occurs gradually over a parameter interval, in contrast to the classical transition which occurs at a specific parameter value. This is seen both for the energy splitting and probability distribution above, but we will see further examples below. The transition interval is however decreasing with increasing $N[166,207]$.

Aubry et al [209] studied some different quantum signatures of the dimer's selftrapping transition, which are not directly associated with the ground state of the system. They found a clear increase in the density of states at the energy of the separatrix (appearing at the self-trapping transition) which divides the classical phase space between trajectories that are symmetric and asymmetric, respectively, under site permutation. There was also a clear decrease in the energy splitting between corresponding quantum eigenstates ${ }^{11}$ with energies above the classical separatrix, which are associated with symmetry-broken trajectories, compared to those with energies below the classical separatrix, which instead are associated with symmetric trajectories.

For the symmetric trimer (periodic boundary conditions), similar to the dimer, the ground state's probability distribution over Fock states makes a transition from single peaked around $|N / 3, N / 3, N / 3\rangle$, to triple peaked around the states $\hat{T}^{j}|N / 3+2 d, N / 3-d, N / 3-d\rangle, j=0,1,2$, where $d$ again increases with the attractive interaction strength [210]. Quantum signatures for the different bifurcation scenarios of the on-site symmetric DB for $3 \leq L \leq 5$ and $L>5$, respectively (see Sec. 3.7), were also observed in Ref. [210 $]^{12}$. By introducing a measure of the Bose-Hubbard ground state's width in the lattice, they showed that (for $N \sim 100$ ) this quantity increases rapidly to the maximal width for $3 \leq L \leq 5$ at parameter values close to the classical self-trapping transition, while a more gradual increase occurs for $L>5$.

Jack and Yamashita [205] studied an attractive Bose-Hubbard ring with $N=$ 10 particles in a $L=6$ site periodic lattice and argued that by increasing the value of $|U / J|$, there is a transition from the superfluid regime (see section 4.9) to a soliton regime, occurring approximately at (3.35) (with the corresponding quantum parameters). They identified the soliton state e.g. with increased values of $L\left\langle\left(\hat{a}_{j}^{\dagger}\right)^{N}\left(\hat{a}_{j+1}\right)^{N}\right\rangle / N$ ! and decreased values of $\left\langle\hat{a}_{j}^{\dagger} \hat{a}_{j+1}\right\rangle$. Buonsante et al [175] used the same model but with up to $N=10$ particles in a $L=20$ site periodic lattice (at most 10 particles on 12 sites or 5 particles on 20 sites). They argued that the soliton regime in [205] actually could be divided into two regimes: a soliton

\footnotetext{
${ }^{11}$ The two eigenstates that can be followed from $|N-n, n\rangle \pm|n, N-n\rangle$, respectively, from the limit $J=0$.

${ }^{12}$ Quantum Monte Carlo calculations where used on the larger systems in Ref. [210].
} 
regime for intermediate $|U / J|$ and a Schrödinger-cat state regime for large $|U / J|$, where the Schrödinger-cat state is defined as being localized on essentially one site $^{13}$. They also argued that the observables that [205] studied actually indicated a transition, not from the superfluid to soliton regime, but from the soliton to the Schrödinger-cat state regime, but since the system considered in [205] was so small, these transitions occur very close to each other [175]. They tested their hypothesis but studying how the different quantities depend on the number of sites, noting that the transition into the Schrödinger-cat regime is independent of the lattice size $L$ and can be estimated with $|J / U(N-1)| \approx 1 / 4$, while the transition into the soliton regime is size dependent, since the size of the lattice limits how broad a soliton can be, and is approximated with Eq. (3.35).

Regarding instead few particles in a large lattice, Scott et al [167] considered the case of two particles in a large one-dimensional, attractive Bose-Hubbard model (4.6). The system's energy spectrum consists of a quasi-continuum, and below that a single band. They called this band the soliton band and the eigenstates in this band has the highest probability for the two bosons to be located close to each other. The energy of the soliton band is in the limit $L \rightarrow \infty$ given by [167]

$$
E=\sqrt{1+16\left(\frac{J}{U}\right)^{2} \cos \left(\frac{k}{2}\right)} .
$$

To create a state for which, at least initially, there is a high probability of finding the two bosons close together, one would take a superposition of the eigenstates in the soliton band. This state would eventually spread due to the energy differences between the eigenstates. Note that the width of the soliton band decreases with increasing magnitude of $U$, and that the delocalization time thus also will increase.

\subsubsection{Quantum Lattice Compactons}

In paper I and III we studied quantum versions of the classical lattice compactons [34] (see Sec. 3.7.2) in four-site lattices. Since the compactons are decaying so rapidly, the four-site lattice can be assumed to be a good approximation also for a larger lattice. This made it possible to use quite many particles (up to 30) and thus better connect with the classical limit.

An attractive model was used in paper III, while paper I used a repulsive one, but these two models can however be mapped on each other, since a staggering transformation together with the parameter substitution $Q_{i} \mapsto-Q_{i}, i=1,3,4$, results in $\hat{H} \mapsto-\hat{H}$ in $(4.26)$.

In paper I the concept of an $m$-site quantum lattice compacton (QLC) eigenstate was introduced, which was defined as an eigenstate with absolute certainty of finding all particles located on $m$ consecutive sites. It was found that onesite QLC eigenstates actually exist and correspond to the classical one-site lattice compacton. They exist under certain parameter conditions, which when fulfilled

\footnotetext{
${ }^{13}$ This is comparable to a one-site compacton, but created by a fundamentally different mechanism than in paper I and III. This can be compared to the difference between a compacton in the anti-continuous limit of the DNLS equation and the compacton created by canceling the coupling with carefully chosen parameter values in the extended DNLS equation.
} 
completely cancels the tunneling from a site with all $N$ particles to its empty neighbors. For these eigenstates the particles can actually be localized on specific sites.

Classical several-site compactons on the other hand do not correspond to exact QLC eigenstates. They correspond instead to superpositions of eigenstates, and it was shown that these eigenstates will become more and more 'QLC-like' as the number of particles increases, i.e. the probability of finding all particles on $m$ consecutive sites goes towards unity.

In paper III we studied how well the dynamical properties of the classical lattice compactons could be reproduced in the extended Bose-Hubbard model, with special emphasis on the mobility. We used two different types of states in the calculations and simulations in paper III: both what we called 'localized ground states' which are superpositions of the lowest energy eigenstates from each quasi-momentum space, and also $\mathrm{SU}(L)$ coherent states given by (4.39). With the help of these states, it was possible for us to clearly distinguish the classical stability regimes of the extended DNLS model [34] by looking at quantities such as the average values of number operators $\left\langle\hat{n}_{j}\right\rangle$ and density correlation functions $\left\langle\hat{n}_{j} \hat{n}_{j+1}\right\rangle$. We then proceeded by studying the dynamics and mobility of $\operatorname{SU}(L)$ coherent states with an applied phase gradient. The main conclusion was that it is possible to see clear signatures of a mobile localized structure, but that this is strongly dependent on the magnitude of the applied phase gradient. For a small phase gradient (cf. Sec. 3.7.1), which classically corresponds to a slow coherent movement of the compacton, the time to travel one site is of the same order as the delocalization time and one can therefore not distinguish any clear signs of the mobility. For a larger phase gradient on the other hand, corresponding to a harder kick and a faster movement, the time-scales separate and it is possible to see a localized profile traveling several sites of the lattice before quantum fluctuations destroy it.

\subsection{Quantum Signatures of Instabilities}

When the instability of a fixed point (Sec. 1.5.2) is to be translated to quantum mechanics, another difficulty arises, which, as mentioned in Sec. 1.6, indeed is at the very heart of the 'strangeness' of quantum mechanics. Due to the Heisenberg uncertainty principle, we cannot even talk about a trajectory in phase space, since position and momentum cannot simultaneously be well defined quantities.

But the question that we can ask is: what kind of signatures of a classical instability can one see in the corresponding quantum mechanical model? This question is very much related to the field of quantum chaos. When transferring chaos to quantum mechanics similar difficulties arise as for the instability, since two initial conditions which are too close to each other in phase space cannot be distinguished from each other, due to the Heisenberg uncertainty principle.

We have already discussed studies of quantum signatures of the self-trapping transition in Sec. 4.6, and the modulational instability of current-carrying constant amplitude waves (see Sec. 3.6) has also been studied in $[211,212]$. In both 
cases, quantum mechanics causes a broadening of the bifurcation point, so that the transition instead happens gradually over a critical regime.

Quantum signatures of an oscillatory instability was, to our knowledge, first studied in paper II with the Bose-Hubbard trimer, for the classical SDW solution of the DNLS trimer (see Sec. 3.6). The classical oscillatory instability regime could be related to avoided crossings in the energy spectrum and a strong mixing between a pure SDW quantum state and other eigenstates. Several measures were also constructed which gave clear signatures on the classically unstable regime. There was for instance a clear decrease in the maximum probability, for any eigenstate, of having the particles equally distributed on two sites. Another measure was the total overlap between compact SDW basis states, having the form of $|N / 2, N / 2,0\rangle$ plugged into (4.11), and basis states which are not two-site localized, summed over all eigenstates. This measure showed a pronounced plateau in the classically unstable regime. It was also possible to identify a simple superposition of quantum eigenstates with the classical SDW stationary states in the classically stable regime, while a similar identification in the unstable regime failed to capture essential features of the unstable dynamics. For example, we showed that the dynamics resulting from a small perturbation of these states cannot reproduce the development of an oscillatory instability. The classical unstable dynamics is thus a result of global properties of the eigenstates, rather than of individual eigenstates. By instead using $\mathrm{SU}(3)$ coherent states (4.39) as initial conditions in the dynamical simulations, it was possible to reproduce several features of the transition from stable internal oscillations to oscillatory instabilities. Similar to the other instabilities mentioned above, the quantum transition happens gradually over an interval, while the classical transition occurs in a single bifurcation point.

\subsection{Quantum Discrete Vortices}

Since, as discussed in Secs. 1.4 and 3.8, discrete vortices are connected to the phase differences between sites, it is useful to have a quantum phase operator ${ }^{14}$ when studying quantum signatures. A commonly used convention is the following phase operators [214]

$$
\begin{gathered}
\sin \left(\Delta \theta_{i j}\right)=i K\left(\hat{a}_{j}^{\dagger} \hat{a}_{i}-\hat{a}_{j} \hat{a}_{i}^{\dagger}\right), \\
\cos \left(\Delta \theta_{i j}\right)=K\left(\hat{a}_{j}^{\dagger} \hat{a}_{i}+\hat{a}_{j} \hat{a}_{i}^{\dagger}\right),
\end{gathered}
$$

where $\Delta \theta_{i j}=\theta_{j}-\theta_{i}$ is the phase difference between sites $j$ and $i$ and the constant $K=1 / \sqrt{2\left\langle 2 \hat{n}_{i} \hat{n}_{j}+\hat{n}_{i}+\hat{n}_{j}\right\rangle}$ is determined from the condition that

$$
\left\langle\sin ^{2}\left(\Delta \theta_{i j}\right)+\cos ^{2}\left(\Delta \theta_{i j}\right)\right\rangle=1 .
$$

Using the Heisenberg equation with Hamiltonian (4.6), one may also derive a continuity equation for the number operator

$$
\frac{d \hat{n}_{j}}{d t}=\hat{J}_{j-1}-\hat{J}_{j}
$$

\footnotetext{
${ }^{14}$ It is actually far from trivial to define a good, Hermitian phase operator. See e.g. [213] for a discussion and historical account on the development of quantum phase operators.
} 
where

$$
\hat{J}_{j}=i J\left(\hat{a}_{j+1}^{\dagger} \hat{a}_{j}-\hat{a}_{j+1} \hat{a}_{j}^{\dagger}\right)
$$

is the current operator from site $j$ to $j+1$, which is proportional to the sine function (4.51a) (cf. Eq. (3.7)).

Lee et al [215] studied quantum signatures in the repulsive Bose-Hubbard trimer ring of plane-wave vortices $\psi_{j}=\psi_{0} \exp ( \pm 2 \pi i j / 3)$. The main conclusion of this work is that quantum fluctuations 'melt' the classical discrete vortices as the interaction strength is increased. This is e.g. manifested in an increase of the fluctuations of the cosine phase operator $(4.51 \mathrm{~b})$. The expectation value of $(4.51 \mathrm{~b})$ will also deviate from the corresponding classical value of 0.5 in this limit. It is possible to identify a single eigenstate with a classical discrete vortex in the weak interaction limit. When this eigenstate is followed adiabatically to the strong interaction limit, it converges to the state $\sum_{j} e^{ \pm 2 \pi i j / 3} \hat{T}^{j}|N / 2, N / 2,0\rangle$, which does not represent any flow of particles [215].

Arwas et al [216] studied quantum discrete vortices in rotating rings. By studying the eigenstates probability distribution over the Fock states, they found that some eigenstates show clear signatures of certain types of 'exotic' discrete vortex states (i.e. not a plane-wave vortex). One example was a 'breathing vortex state', which is a (quasi-)periodic vortex (of type (3.12)), similar to the ones shown in Fig. 5 in Paper IV. Another example was eigenstates which correspond to classical vortices which are chaotic.

In Paper VI we studied quantum signatures of the charge flipping vortices of the DNLS trimer (see Sec. 3.8). This work focused mainly on the dynamical properties of the $\mathrm{SU}(3)$ coherent states obtained by plugging the classical charge flipping vortices $\psi_{j}$ into Eq. (4.39). Since these classical charge flipping vortices are not stationary solutions, using $\psi_{j}(t)$ with different $t$ generate different coherent states, and we mostly chose a $t$ when the charge flipped. We observed that the quantum average of the number operator $\left\langle\hat{n}_{j}\right\rangle$ follows the classical $\left|\psi_{j}\right|^{2}$ better the closer the charge flipping vortex is to the stable dimerlike solution. Since vortices are characterized by the flows, we also considered the average values of the current operators. The three currents (between the different sites) generally pointed in the same rotational direction and flipped at approximately the same time, even in the regimes where $\left\langle\hat{n}_{j}\right\rangle$ and $\left|\psi_{j}\right|^{2}$ diverge from each other, which shows that the charge flipping vortices are rather robust in this sense. In analogy with (1.26), we also introduced a quantum topological charge, calculated from the phase operators (4.51), which indeed indicated the direction of the current, even though it sometimes took classically forbidden non-integer values due to quantum fluctuations in the phase. Changing $t$ in $\psi_{j}(t)$ did not alter the time evolution of the coherent states much for charge flipping vortices close to the dimerlike solution. For $\psi_{j}(t)$ closer to the SDW solution it had a greater impact, e.g. spreading out the projection of the coherent states over more eigenstates. This caused a stronger deviation of $\left\langle\hat{n}_{j}\right\rangle$ from $\left|\psi_{j}\right|^{2}$, and could also destroy the charge flipping seen in the average currents. 


\subsection{Superfluid to Mott Insulator Transition}

The superfluid to Mott insulator transition is not something that any of the Papers I-VI covers, but since it is one of the most important, and most studied, concepts for Bose-Hubbard models and BECs in optical lattices, we briefly discuss it.

For repulsive interactions, the Bose-Hubbard model (4.1) undergoes a quantum phase transition from a superfluid to Mott insulating state [217]. This transition was first discussed for the Bose-Hubbard model in a paper by Fisher at al [218], but with systems such as ${ }^{4} \mathrm{He}$ absorbed in porous media and granular superconductors in mind. It was in another seminal paper by Jaksch et al [219] where it was proposed, first of all that the Bose-Hubbard model should be applicable for ultracold atoms in optical lattices, but also that the transition could be realizable with such a system. This was experimentally observed in 2002 by Greiner et al [85] in a three-dimensional lattice, and has since also been observed in both one- and two-dimensional lattices [220,221].

The transition illustrates the competition between the repulsive on-site interaction and kinetic energy, so that when the interaction energy 'wins', the ground state is a Mott insulator, while it becomes a superfluid when the kinetic energy prevails. To get some insight into the nature of these two states, it is instructive to look at the form the ground states take for a homogeneous Bose-Hubbard model (4.1) with $N$ particles in a $\mathcal{L}$-site lattice, in the two limits $U \gg J$ and $U \ll J$.

The ground state is in the noninteracting limit $(U=0)$ given by

$$
\left|\Psi_{S F}(N)\right\rangle_{(U=0)}=\frac{1}{\sqrt{N !}}\left(\frac{1}{\sqrt{\mathcal{L}}} \sum_{\mathbf{R}} \hat{a}_{\mathbf{R}}^{\dagger}\right)^{N}|0, \ldots, 0\rangle,
$$

which is a $\mathrm{SU}(\mathcal{L})$ coherent state $(4.39)$ with $\psi_{\mathbf{R}}=\sqrt{N / \mathcal{L}}$. Equation (4.55) is a pure BEC with all $N$ particles in the quasimomentum state $\mathbf{q}=0$ (c.f. (4.11)). The atoms are now in a superfluid phase [218], where each atom is completely delocalized, and allowed to move freely over the whole lattice.

In the thermodynamic limit, $N, \mathcal{L} \rightarrow \infty$ with fixed density $N / \mathcal{L}$, state $(4.55)$ can be approximated as a product of Glauber coherent states for each lattice site $\mathbf{R}$, with an average particle occupation $\left\langle\hat{n}_{j}\right\rangle=N / \mathcal{L}$,

$$
\left|\Psi_{S F}(N \rightarrow \infty)\right\rangle_{(U=0)} \approx \exp \left(\sqrt{\frac{N}{\mathcal{L}}} \sum_{\mathbf{R}} \hat{a}_{\mathbf{R}}^{\dagger}\right)|0, \ldots, 0\rangle=\prod_{\mathbf{R}} \exp \left(\sqrt{\frac{N}{\mathcal{L}}} \hat{a}_{\mathbf{R}}^{\dagger}\right)|0, \ldots, 0\rangle
$$

In the opposite limit, $J=0$, tunneling is completely suppressed. The repulsive interaction energy will also try to reduce the number of particles of each site as much as possible. Considering at first a system with commensurate particle filling, i.e. $\tilde{n}=N / \mathcal{L}$ is an integer, this means that the ground state will have the particles evenly spread out, i.e. exactly $\tilde{n}$ particles on each site,

$$
\left|\Psi_{M I}(\tilde{n})\right\rangle_{(J=0)}=\left(\prod_{\mathbf{R}} \frac{\left(\hat{a}_{\mathbf{R}}^{\dagger}\right)^{\tilde{n}}}{\sqrt{\tilde{n}}}\right)|0, \ldots, 0\rangle
$$


This is a Mott insulator [218]. By tunneling a particle, so that one site has $\tilde{n}+1$ bosons and another $\tilde{n}-1$, the energy is increased with $U$. There can therefore not be a flow of particles over the lattice in this state, and the bosons thus are localized to specific sites. The site with $\tilde{n}+1$ bosons can be referred to as a 'particle' and the one with $\tilde{n}-1$ as a 'hole', in analogy with Dirac's electron sea. Note also that the Mott insulator is not a BEC, and it is therefore expected that mean-field descriptions will fail to describe this regime.

Even though the ground state only takes the simple product forms of (4.55) and (4.57) in these specific limits, classifications as Mott insulator or superfluid is valid also in the intermediate regime [76].

To illustrate the mechanism behind the phase transition, consider what will happen to state (4.57) when $J$ is being turned up. Should an atom now hop from one site to the next, then there would on one hand be a gain of kinetic energy of order $J$, but also a cost in interaction energy for creating a 'hole' and a 'particle', which is of order $U$. Thus, if $J$ is much smaller than $U$, hopping is energetically unfavorable, and the atoms will stay localized on the sites, thus still in the Mott insulating phase. But when $J$ becomes of the same order as $U$ the cost in interaction energy can be outweighed by the gain in kinetic energy and it will thus be beneficial to create an electron-hole pair. Also, as soon as the particle and hole have been created, they will move freely over the lattice, since they are moving over a constant background (neglecting the effect that the particle and hole have on each other) and there is no cost in interaction energy for the particle or hole to move between sites with the same number of particles, therefore making the state superfluid [76].

What will happen if there is not a commensurate filling? Imagine that a single atom is added to the Mott insulating phase discussed above. This atom would, just as the particle and hole, be able to move freely over the constant background, and it will thereby be in the superfluid state all the way down to $J=0$. This reasoning might suggest that the Mott insulating phase would be extremely hard to realize, but remember that the discussion so far has been for a homogeneous system. By performing the experiments in a slowly varying harmonic trap, leading to a spatially varying on-site energy, one would observe that different regions of the lattice are in the Mott insulating phase, each with different number of particles per site, and that these regions are separated by superfluid regions [219].

The two states obviously differ in many aspects. They for instance exhibit very different phase coherences, which can be understood on the basis of the Heisenberg uncertainty relation for phase and number of atoms at a site, i.e. if the number of atoms on a site is well specified the phase is uncertain, preventing phase coherence between sites, and vice versa. It is therefore low phase coherence (actually none for state (4.57)) between different sites in the Mott insulator phase, but long range phase coherence in the superfluid phase. This difference can actually be utilized to experimentally test the transition. When atoms in the superfluid phase are released from the optical lattice (this is done by simply turning the lasers off) they will lump together in clear interference peaks because of the long range phase coherence. The crossover to the Mott insulating phase can thus be identified, essentially by looking at when these interference peaks disappear [85]. 
Another important difference is in the excitation spectrum, where the Mott insulator has a finite energy gap corresponding to the creation of a particle-hole pair. There is on the other hand no energy gap for the superfluid phase, which instead has sound-like excitations, with a linear relation between frequency and wave number. The energy gap in the Mott insulating phase has been experimentally verified $[85,220]$. 


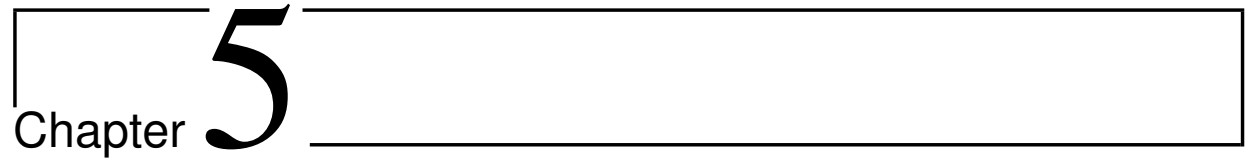

\section{Concluding Comments}

This chapter concludes the first part of the thesis, where the goal has been to introduce the reader to certain models and notions associated with nonlinear science - primarily those that I have been studying during my time as a PhD-student. The rest of the thesis contains six papers which is the main result of my work as a PhD-student. Certain results from these have already been discussed in the previous chapters, with the purpose of putting them in a relevant context. The papers can be divided into two categories.

Papers IV and V are concerned with studies of certain types of solutions to DNLS models. Paper IV deals with charge flipping vortices in DNLS trimers and hexamers, while Paper V deals with discrete breathers in DNLS rings with a central site.

Papers I-III and VI are on the other hand concerned with Bose-Hubbard models, and of studying quantum signatures of results which have been obtained for corresponding DNLS models. Papers I and III deal with lattice compactons in an extended Bose-Hubbard model, where Paper I is concerned with the eigenstates that correspond to the classical lattice compactons, and Paper III focuses on the dynamics of certain quantum states. Paper II is instead focused on quantum signatures of the oscillatory instability, in the standard Bose-Hubbard model, which a solution (the single-depleted well state) of the DNLS trimer experiences. Finally, Paper VI studies quantum signatures of the charge flipping vortices of Paper IV (for the trimer). 


\section{Bibliography}

[1] A.C. Scott. The Nonlinear Universe. Springer-Verlag, Berlin Heidelberg, 2007.

[2] S.H. Strogatz. Nonlinear Dynamics and Chaos: With Applications to Physics, Biology, Chemistry, and Engineering. Westview Press, Boulder, CO, second edition, 2015.

[3] A. Scott. Nonlinear Science - Emergence and Dynamics of Coherent Structures. Oxford University Press, Oxford, second edition, 2003.

[4] G. Ohlén, S. Åberg, and P. Östborn. Compendium "Chaos" and references therein. Division of Mathematical Physics LTH, Lund, 2007.

[5] N.W. Ashcroft and N.D. Mermin. Solid State Physics. Brooks/Cole, Cengage Learning, Belmont, 1976.

[6] E. Fermi, J. Pasta, and S. Ulam. Studies of non Linear Problems. Los Alamos Scientific Laboratory Report No. La-1940, 1955.

[7] G. Gallavotti, editor. The Fermi-Pasta-Ulam Problem - A Status Report. Springer-Verlag, Berlin Heidelberg, 2008.

[8] M.J. Ablowitz and Z.H. Musslimani. Integrable nonlocal nonlinear Schrödinger equation. Physical Review Letters, 110:064105, 2013.

[9] C.J. Pethick and H. Smith. Bose-Einstein Condensation in Dilute Gases. Cambridge University Press, Cambridge, second edition, 2008.

[10] D.J. Korteweg and G. de Vries. On the change of form of long waves advancing in a rectangular canal, and on a new type of long stationary waves. Philosophical Magazine, 39:422, 1895.

[11] J.S. Russell. Reports on Waves. British Association for the Advancement of Science, 1845. 
[12] N.J. Zabusky and M.D. Kruskal. Interaction of solitons in a collisionless plasma and the recurrence of initial states. Physical Review Letters, 15:240, 1965.

[13] M. Tabor. Chaos and Integrability in Nonlinear Dynamics - An Introduction. John Wiley \& Sons, New York, 1989.

[14] M. Remoissenet. Waves Called Solitons - Concepts and Experiments. Springer-Verlag, Berlin Heidelberg, 3 edition, 1999.

[15] S. Aubry. Discrete Breathers: Localization and transfer of energy in discrete Hamiltonian nonlinear systems. Physica D, 216:1, 2006.

[16] S. Flach and A.V. Gorbach. Discrete breathers - Advances in theory and applications. Physics Reports, 467:1, 2008.

[17] R.S. MacKay and S. Aubry. Proof of existence of breathers for time-reversible or Hamiltonian networks of weakly coupled oscillators. Nonlinearity, 7:1623, 1994.

[18] J.-A. Sepulchre and R.S. MacKay. Localized oscillations in conservative or dissipative networks of weakly coupled autonomous oscillators. Nonlinearity, 10:679, 1997.

[19] J.L. Marín and S. Aubry. Breathers in nonlinear lattices: numerical calculation from the anticontinuous limit. Nonlinearity, 9:1501, 1996.

[20] P. Binder, D. Abraimov, A.V. Ustinov, S. Flach, and Y. Zolotaryuk. Observation of Breathers in Josephson Ladders. Physical Review Letters, 84:745, 2000 .

[21] E. Trías, J.J. Mazo, and T.P. Orlando. Discrete Breathers in Nonlinear Lattices: Experimental Detection in a Josephson Array. Physical Review Letters, 84:741, 2000.

[22] A.V. Ustinov. Imaging of discrete breathers. Chaos, 13:716, 2003.

[23] B.I. Swanson, J.A. Brozik, S.P. Love, G.F. Strouse, A.P. Shreve, A.R. Bishop, W.-Z. Wang, and M.I. Salkola. Observation of Intrinsically Localized Modes in a Discrete Low-Dimensional Material. Physical Review Letters, 82:3288, 1999.

[24] U.T. Schwarz, L.Q. English, and A.J. Sievers. Experimental Generation and Observation of Intrinsic Localized Spin Wave Modes in an Antiferromagnet. Physical Review Letters, 83:223, 1999.

[25] M. Sato and A.J. Sievers. Direct observation of the discrete character of intrinsic localized modes in an antiferromagnet. Nature, 432:486, 2004. 
[26] M. Sato, B.E. Hubbard, A.J. Sievers, B. Ilic, D.A. Czaplewski, and H.G. Craighead. Observation of Locked Intrinsic Localized Vibrational Modes in a Micromechanical Oscillator Array. Physical Review Letters, 90:044102, 2003.

[27] M. Sato, B.E. Hubbard, L.Q. English, A.J. Sievers, B. Ilic, D.A. Czaplewski, and H.D Craighead. Study of intrinsic localized vibrational modes in micromechanical oscillator arrays. Chaos, 13:702, 2003.

[28] M. Sato, B.E. Hubbard, A.J. Sievers, B. Ilic, and H.G. Craighead. Optical manipulation of intrinsic localized vibrational energy in cantilever arrays. Europhysics Letters, 66:318, 2004.

[29] R. Basu Thakur, L.Q. English, and A.J. Sievers. Driven intrinsic localized modes in a coupled pendulum array. Journal of Physics D: Applied Physics, 41:015503, 2008.

[30] J. Cuevas, L.Q. English, P.G. Kevrekidis, and M. Anderson. Discrete Breathers in a Forced-Damped Array of Coupled Pendula: Modeling, Computation, and Experiment. Physical Review Letters, 102:224101, 2009.

[31] P. Rosenau and J.M. Hyman. Compactons: Solitons with Finite Wavelength. Physical Review Letters, 70:564, 1993.

[32] P.G. Kevrekidis and V.V. Konotop. Bright compact breathers. Physical Review E, 65:066614, 2002.

[33] P.G. Kevrekidis and V.V. Konotop. Compactons in discrete nonlinear KleinGordon models. Mathematics and Computers in Simulation, 62:79, 2003.

[34] M. Öster, M. Johansson, and A. Eriksson. Enhanced mobility of strongly localized modes in waveguide arrays by inversion of stability. Physical Review E, 67:056606, 2003.

[35] F.Kh. Abdullaev, P.G. Kevrekidis, and M. Salerno. Compactons in Nonlinear Schrödinger Lattices with Strong Nonlinearity Management. Physical Review Letters, 105:113901, 2010.

[36] J. D’Ambroise, M. Salerno, P.G. Kevrekidis, and F.Kh. Abdullaev. Multidimensional discrete compactons in nonlinear Schrödinger lattices with strong nonlinearity management. Physical Review A, 92:053621, 2015.

[37] M. Öster, Y.B. Gaididei, M. Johansson, and P.L. Christiansen. Nonlocal and nonlinear dispersion in a nonlinear Schrödinger-type equation: exotic solitons and short-wavelength instabilities. Physica D: Nonlinear Phenomena, 198:29, 2004.

[38] M. Eleftheriou, B. Dey, and G.P. Tsironis. Compactlike breathers: Bridging the continuous with the anticontinuous limit. Physical Review E, 62:7540, 2000 . 
[39] B. Dey, M. Eleftheriou, S. Flach, and G.P. Tsironis. Shape profile of compactlike discrete breathers in nonlinear dispersive lattice systems. Physical Review E, 65:017601, 2002.

[40] P. Rosenau and S. Schochet. Almost Compact Breathers in Anharmonic Lattices near the Continuum Limit. Physical Review Letters, 94:045503, 2005.

[41] P. Tchofo Dinda and M. Remoissenet. Breather compactons in nonlinear Klein-Gordon systems. 60:6218, 1999.

[42] D.L. Bergman, C. Wu, and L. Balents. Band touching from real-space topology in frustrated hopping models. Physical Review B, 78:125104, 2008.

[43] A.S. Desyatnikov, Y.S. Kivshar, and L. Torner. Optical vortices and vortex solitons. Progress in Optics, 47:291, 2005.

[44] J. Yang and Z.H. Musslimani. Fundamental and vortex solitons in a twodimensional optical lattice. Optics Letters, 28:2094, 2003.

[45] J. Yang. Stability of vortex solitons in a photorefractive optical lattice. New Journal of Physics, 6:47, 2004.

[46] T.J. Alexander, A.A. Sukhorukov, and Y.S. Kivshar. Asymmetric Vortex Solitons in Nonlinear Periodic Lattices. Physical Review Letters, 93:063901, 2004.

[47] T. Cretegny and S. Aubry. Spatially inhomogeneous time-periodic propagating waves in anharmonic systems. Physical Review B, 55:R11929, 1997.

[48] M. Johansson, S. Aubry, Y.B. Gaididei, P.L. Christiansen, and K.Ø. Rasmussen. Dynamics of breathers in discrete nonlinear Schrödinger models. Physica D, 119:115, 1998.

[49] B.A. Malomed and P.G. Kevrekidis. Discrete vortex solitons. Physical Review E, 64:026601, 2001.

[50] P.G. Kevrekidis, B.A. Malomed, and Yu.B. Gaididei. Solitons in triangular and honeycomb dynamical lattices with the cubic nonlinearity. Physical Review E, 66:016609, 2002.

[51] D.E. Pelinovsky, P.G. Kevrekidis, and D.J. Frantzeskakis. Persistence and stability of discrete vortices in nonlinear Schrödinger lattices. Physica D, 212:20, 2005.

[52] M. Öster and M. Johansson. Stable stationary and quasiperiodic discrete vortex breathers with topological charge $\mathrm{S}=2$. Physical Review E, 73:066608, 2006.

[53] A.S. Desyatnikov, M.R. Dennis, and A. Ferrando. All-optical discrete vortex switch. Physical Review A, 83:063822, 2011. 
[54] D. Leykam and A.S. Desyatnikov. Discrete multivortex solitons. Optics Letters, 36:4806, 2011.

[55] D. Leykam and A.S. Desyatnikov. Vortex switching with discrete multivortex solitons. Physical Review A, 86:043812, 2012.

[56] D. Leykam, V.V. Konotop, and A.S. Desyatnikov. Discrete vortex solitons and parity time symmetry. Optics Letters, 38:371, 2013.

[57] G. Gibson, J. Courtial, M.J. Padgett, M. Vasnetsov, V. Pas'ko, S. Barnett, and S. Franke-Arnold. Free-space information transfer using light beams carrying orbital angular momentum. Optics Express, 12:5448, 2004.

[58] M.C. Gutzwiller. Chaos in Classical and Quantum Mechanics. SpringerVerlag, New York, 1990.

[59] H. Goldstein, C. Poole, and J. Safko. Classical Mechanics. Pearson Education Interational, Upper Saddle River, N.J., third edition, 2002.

[60] C. Robinson. Dynamical Systems. CRC Press, Boca Raton, second edition, 1999.

[61] M. Johansson. Hamiltonian Hopf bifurcations in the discrete nonlinear Schrödinger trimer: oscillatory instabilities, quasi-periodic solutions and a 'new' type of self-trapping transition. Journal of Physics A: Mathematical and General, 37:2201, 2004.

[62] P. Cvitanović, R. Artuso, R. Mainieri, G. Tanner, and G. Vattay. Chaos: Classical and Quantum. ChaosBook.org. Niels Bohr Institute, Copenhagen, 2016.

[63] E. Merzbacher. Quantum Mechanics. John Wiley \& Sons, Inc., Hoboken, third edition, 1998.

[64] R Loudon. The Quantum Theory of Light. OUP Oxford, Oxford, third edition, 2000.

[65] S.N. Bose. Plancks Gesetz und Lichtquantenhypothese. Zeitschrift für Physik, 26:178, 1924.

[66] A. Einstein. Quantentheorie des einatomigen idealen Gases. Sitzber. Kgl. Preuss. Akad. Wiss., 261, 1924.

[67] A. Einstein. Quantentheorie des einatomigen idealen Gases. 2. Abhandlung. Sitzber. Kgl. Preuss. Akad. Wiss., 3, 1925.

[68] F. London. The $\lambda$-Phenomenon of Liquid Helium and the Bose-Einstein Degeneracy. Nature, 141:643, 1938.

[69] L. Pitaevskii and S. Stringari. Bose-Einstein Condensation. Claderon Press, Oxford, 2003. 
[70] C.E. Hecht. The Possible Superfluid Behaviour of Hydrogen Atom Gases and Liquids. Physica, 25:1159, 1959.

[71] W.C. Stwalley and L.H. Nosanow. Possible "New" Quantum Systems. Physical Review Letters, 36:910, 1976.

[72] M.H. Anderson, J.R. Ensher, M.R. Matthews, C.E. Wieman, and E.A. Cornell. Observation of Bose-Einstein Condensation in a Dilute Atomic Vapor. Science, 269:198, 1995.

[73] C.C. Bradley, C.A. Sackett, J.J. Tollett, and R.G. Hulet. Evidence of BoseEinstein Condensation in an Atomic Gas with Attractive Interactions. Physical Review Letters, 75:1687, 1995.

[74] K.B. Davis, M.-O. Mewes, M.R. Andrews, N.J. van Druten, D.S. Durfee, D.M. Kurn, and W. Ketterle. Bose-Einstein Condensation in a Gas of Sodium Atoms. Physical Review Letters, 75:3969, 1995.

[75] D.G. Fried, T.C. Killian, L. Willmann, D. Landhuis, S.C. Moss, D. Kleppner, and T.J. Greytak. Bose-Einstein Condensation of Atomic Hydrogen. Physical Review Letters, 81:3811, 1998.

[76] I. Bloch, J. Dalibard, and W. Zwerger. Many-body physics with ultracold gases. Reviews of Modern Physics, 80:885, 2008.

[77] F. Dalfovo, S. Giorgini, L.P. Pitaevskii, and S. Stringari. Theory of BoseEinstein condensation in trapped gases. Reviews of Modern Physics, 71:463, 1999.

[78] S. Burger, K. Bongs, S. Dettmer, W. Ertmer, K. Sengstock, A. Sanpera, G.V. Shlyapnikov, and M. Lewenstein. Dark Solitons in Bose-Einstein Condensates. Physical Review Letters, 83:5198, 1999.

[79] J. Denschlag, J.E. Simsarian, D.L. Feder, C.W. Clark, L.A. Collins, J. Cubizolles, L. Deng, E.W. Hagley, K. Helmerson, W.P. Reinhardt, S.L. Rolston, B.I. Schneider, and W.D. Phillips. Generating Solitons by Phase Engineering of a Bose-Einstein Condensate. Science, 287:97, 2000.

[80] L. Khaykovich, F. Schreck, G. Ferrari, T. Bourdel, J. Cubizolles, L.D. Carr, Y. Castin, and C. Salomon. Formation of a matter-wave bright soliton. Science, 296:1290, 2002.

[81] K.E. Strecker, G.B. Partridge, A.G. Truscott, and R.G. Hulet. Formation and propagation of matter-wave soliton trains. Nature, 417:150, 2002.

[82] M.R. Matthews, B.P. Anderson, P.C. Haljan, D.S. Hall, C.E. Wieman, and E.A. Cornell. Vortices in a Bose-Einstein Condensate. Physical Review Letters, 83:2498, 1999.

[83] O. Morsch and M. Oberthaler. Dynamics of Bose-Einstein condensates in optical lattices. Reviews of Modern Physics, 78:179, 2006. 
[84] P. Windpassinger and K. Sengstock. Engineering novel optical lattices. Reports on progress in physics. Physical Society (Great Britain), 76:086401, 2013.

[85] M. Greiner, O. Mandel, T. Esslinger, T.W. Hänsch, and I. Bloch. Quantum phase transition from a superfluid to a Mott insulator in a gas of ultracold atoms. Nature, 415:39, 2002.

[86] I. Bloch, J. Dalibard, and S. Nascimbène. Quantum simulations with ultracold quantum gases. Nature Physics, 8:267, 2012.

[87] B. Eiermann, Th. Anker, M. Albiez, M. Taglieber, P. Treutlein, K.-P. Marzlin, and M.K. Oberthaler. Bright Bose-Einstein Gap Solitons of Atoms with Repulsive Interaction. Physical Review Letters, 92:230401, 2004.

[88] Th. Anker, M. Albiez, R. Gati, S. Hunsmann, B. Eiermann, A. Trombettoni, and M.K. Oberthaler. Nonlinear Self-Trapping of Matter Waves in Periodic Potentials. Physical Review Letters, 94:020403, 2005.

[89] J.D. Jackson. Classical Electrodynamics. John Wiley \& Sons, New York, third edition, 1999.

[90] A. Yariv. Coupled-Mode Theory for Guided-Wave Optics. IEEE Journal of Quantum Electronics, 9:919, 1973.

[91] J. Kerr. A new Relation between Electricity and Light: Dielectrified Media Birefringent. Philosophical Magazine and Journal of Science, 50:337, 1875.

[92] R.W. Boyd. Nonlinear Optics. Academic Press, San Diego, second edition, 2003.

[93] P.A. Franken, A.E. Hill, C.W. Peters, and G. Weinreich. Generation of optical harmonics. Physical Review Letters, 7:118, 1961.

[94] T.H. Maiman. Stimulated Optical Radiation in Ruby. Nature, 187:493, 1960.

[95] A. Szameit, F. Dreisow, T. Pertsch, S. Nolte, and A. Tünnermann. Control of directional evanescent coupling in fs laser written waveguides. Optics Express, 15:1579, 2007.

[96] P. Miller, J.S. Aitchison, J.U. Kang, G.I. Stegeman, A. Villeneuve, G.T. Kennedy, and W. Sibbett. Nonlinear waveguide arrays in AlGaAs. Journal of the Optical Society of America B, 14:3224, 1997.

[97] N.K. Efremidis, S. Sears, D.N. Christodoulides, J.W. Fleischer, and M. Segev. Discrete solitons in photorefractive optically induced photonic lattices. Physical Review E, 66:046602, 2002.

[98] K. Itoh, W. Watanabe, S. Nolte, and C..B. Schaffer. Ultrafast Processes for Bulk Modification of Transparent Materials. MRS Bulletin, 31:620, 2006. 
[99] D.N. Christodoulides and R.I. Joseph. Discrete self-focusing in nonlinear arrays of coupled waveguides. Optics Letters, 13:794, 1988.

[100] H.S. Eisenberg, Y. Silberberg, R. Morandotti, A.R. Boyd, and J.S. Aitchison. Discrete Spatial Optical Solitons in Waveguide Arrays. Physical Review Letters, 81:3383, 1998.

[101] J.W. Fleischer, T. Carmon, M. Segev, N.K. Efremidis, and D.N. Christodoulides. Observation of Discrete Solitons in Optically Induced Real Time Waveguide Arrays. Physical Review Letters, 90:023902, 2003.

[102] A. Szameit, D. Blömer, J. Burghoff, T. Schreiber, T. Pertsch, S. Nolte, A. Tünnermann, and F. Lederer. Discrete nonlinear localization in femtosecond laser written waveguides in fused silica. Optics Letters, 13:10552, 2005.

[103] J.W. Fleischer, M. Segev, N.K. Efremidis, and D.N. Christodoulides. Observation of two-dimensional discrete solitons in optically induced nonlinear photonic lattices. Nature, 422:147, 2003.

[104] A. Szameit, J. Burghoff, T. Pertsch, S. Nolte, and A. Tünnermann. Twodimensional soliton in cubic fs laser written waveguide arrays in fused silica. Optics Express, 14:6055, 2006.

[105] F. Lederer, G.I. Stegeman, D.N. Christodoulides, G. Assanto, M. Segev, and Y. Silberberg. Discrete solitons in optics. Physics Reports, 463:1, 2008.

[106] M. Heinrich, R. Keil, F. Dreisow, A. Tünnermann, A. Szameit, and S. Nolte. Nonlinear discrete optics in femtosecond laser-written photonic lattices. $A p$ plied Physics B, 104:469, 2011.

[107] R. Morandotti, U. Peschel, J.S. Aitchison, H.S. Eisenberg, and Y. Silberberg. Dynamics of Discrete Solitons in Optical Waveguide Arrays. Physical Review Letters, 83:2726, 1999.

[108] D.N. Neshev, T.J. Alexander, E.A Ostrovskaya, Y.S. Kivshar, H. Martin, I. Makasyuk, and Z. Chen. Observation of Discrete Vortex Solitons in Optically Induced Photonic Lattices. Physical Review Letters, 92:123903, 2004.

[109] J.W. Fleischer, G. Bartal, O. Cohen, O. Manela, M. Segev, J. Hudock, and D.N. Christodoulides. Observation of Vortex-Ring "Discrete" Solitons in 2D Photonic Lattices. Physical Review Letters, 92:123904, 2004.

[110] O. Manela, O. Cohen, G. Bartal, J.W. Fleischer, and M. Segev. Twodimensional higher-band vortex lattice solitons. Optics Letters, 29:2049, 2004 .

[111] G. Bartal, O. Manela, O. Cohen, J.W. Fleischer, and M. Segev. Observation of Second-Band Vortex Solitons in 2D Photonic Lattices. Physical Review Letters, 95:053904, 2005. 
[112] A. Bezryadina, E. Eugenieva, and Z. Chen. Self-trapping and flipping of double-charged vortices in optically induced photonic lattices. Optics Letters, 31:2456, 2006.

[113] B. Terhalle, T. Richter, A.S. Desyatnikov, D.N. Neshev, W. Krolikowski, F. Kaiser, C. Denz, and Y.S. Kivshar. Observation of Multivortex Solitons in Photonic Lattices. Physical Review Letters, 101:013903, 2008.

[114] B. Terhalle, T. Richter, K.J.H. Law, D. Göries, P. Rose, T.J. Alexander, P.G. Kevrekidis, A.S. Desyatnikov, W. Krolikowski, F. Kaiser, C. Denz, and Y.S. Kivshar. Observation of double-charge discrete vortex solitons in hexagonal photonic lattices. Physical Review A, 79:043821, 2009.

[115] B. Terhalle, D. Göries, T. Richter, P. Rose, A.S. Desyatnikov, F. Kaiser, and C. Denz. Anisotropy-controlled topological stability of discrete vortex solitons in optically induced photonic lattices. Optics Letters, 35:604, 2010.

[116] F. Diebel, D. Leykam, M. Boguslawski, P. Rose, C. Denz, and A.S. Desyatnikov. All-optical switching in optically induced nonlinear waveguide couplers. Applied Physics Letters, 104:261111, 2014.

[117] M. Johansson. Discrete nonlinear Schrödinger equations. In A. Scott, editor, Encyclopedia of Nonlinear Science, page 213. Routledge, New York, 2005.

[118] T. Holstein. Studies of Polaron Motion. Annals of Physics, 8:325, 1959.

[119] A. Scott. Davydov's soliton. Physics Reports, 217:1, 1992.

[120] A.C. Scott and J.C. Eilbeck. On the CH stretch overtones of benzene. Chemical physics letters, 132:23, 1986.

[121] M. Johansson and S. Aubry. Existence and stability of quasiperiodic breathers in the discrete nonlinear Schrödinger equation. Nonlinearity, 10:1151, 1997.

[122] A. Trombettoni and A. Smerzi. Discrete Solitons and Breathers with Dilute Bose-Einstein Condensates. Physical Review Letters, 86:2353, 2001.

[123] B.P. Anderson and M.A. Kasevich. Macroscopic Quantum Interference from Atomic Tunnel Arrays. Science, 282:1686, 1998.

[124] A. Smerzi and A. Trombettoni. Discrete nonlinear dynamics of weakly coupled Bose-Einstein condensates. Chaos, 13:766, 2003.

[125] A. Eriksson. Studies of nonlinear arrays of coupled waveguides. Master thesis, Linköping University, 1995.

[126] Y.S. Kivshar and M. Peyrard. Modulational instabilities in discrete lattices. Physical Review A, 46:3198, 1992. 
[127] I. Daumont, T. Dauxois, and M. Peyrard. Modulational instability : first step towards energy localization in nonlinear lattices. Nonlinearity, 10:617, 1997.

[128] M. Johansson and Y.S. Kivshar. Discreteness-Induced Oscillatory Instabilities of Dark Solitons. Physical Review Letters, 82:85, 1999.

[129] A. Alvarez, J.F.R. Archilla, J. Cuevas, and F.R. Romero. Dark breathers in Klein-Gordon lattices. Band analysis of their stability properties. New Journal of Physics, 4:1, 2002.

[130] J.L. Marín, S. Aubry, and L.M. Floría. Intrinsic localized modes: Discrete breathers. Existence and linear stability. Physica D, 113:283, 1998.

[131] S. Darmanyan, A. Kobyakov, and F. Lederer. Stability of strongly localized excitations in discrete media with cubic nonlinearity. Journal of Experimental and Theoretical Physics, 86:682, 1998.

[132] P.G. Kevrekidis, A.R. Bishop, and K.Ø. Rasmussen. Twisted localized modes. Physical Review E, 63:036603, 2001.

[133] A.M. Morgante, M. Johansson, G. Kopidakis, and S. Aubry. Oscillatory Instabilities of Standing Waves in One-Dimensional Nonlinear Lattices. Physical Review Letters, 85:550, 2000.

[134] M. Johansson, A.M. Morgante, S. Aubry, and G. Kopidakis. Standing wave instabilities, breather formation and thermalization in a Hamiltonian anharmonic lattice. The European Physical Journal B - Condensed Matter, 29:279, 2002.

[135] A.V. Gorbach and M. Johansson. Discrete gap breathers in a diatomic KleinGordon chain: Stability and mobility. Physical Review E, 67:066608, 2003.

[136] L. Kroon, M. Johansson, A.S. Kovalev, and E.Yu. Malyuta. The appearance of gap solitons in a nonlinear Schrödinger lattice. Physica D, 239:269, 2010.

[137] R. Franzosi and V. Penna. Chaotic behavior, collective modes, and selftrapping in the dynamics of three coupled Bose-Einstein condensates. Physical Review E, 67:046227, 2003.

[138] J. Carr and J.C. Eilbeck. Stability of stationary solutions of the discrete self-trapping equation. Physics Letters A, 109:201, 1985.

[139] J.B. Page. Asymptotic solutions for localized vibrational modes in strongly anharmonic periodic systems. Physical Review B, 41:7835, 1990.

[140] A.J. Sievers and S. Takeno. Intrinsic Localized Modes in Anharmonic Crystals. Physical Review Letters, 61:970, 1988. 
[141] J.C. Eilbeck and M. Johansson. The discrete nonlinear Schrödinger equation - 20 years on. In L. Vásquez, R.S. MacKay, and M.P. Zorzano, editors, Localization 8 Energy Transfer in Nonlinear Systems, page 44. World Scientific, New Jersey, 2003.

[142] P. Buonsante, P. Kevrekidis, V. Penna, and A. Vezzani. Ground-state properties of attractive bosons in mesoscopic 1D ring lattices. Journal of Physics B: Atomic, Molecular and Optical Physics, 39:S77, 2006.

[143] P. Buonsante, P.G. Kevrekidis, V. Penna, and A. Vezzani. Ground-state properties of small-size nonlinear dynamical lattices. Physical Review E, 75:016212, 2007.

[144] J.C. Eilbeck, P.S. Lomdahl, and A.C. Scott. The discrete self-trapping equation. Physica D, 16:318, 1985.

[145] K.Ø. Rasmussen, T. Cretegny, P.G. Kevrekidis, and N. Grønbech-Jensen. Statistical mechanics of a discrete nonlinear system. Physical Review Letters, 84:3740, 2000.

[146] K.Ø. Rasmussen, S. Aubry, A.R. Bishop, and G.P. Tsironis. Discrete nonlinear Schrödinger breathers in a phonon bath. The European Physical Journal $B, 15: 169,2000$.

[147] M Jenkinson and M I Weinstein. Onsite and offsite bound states of the discrete nonlinear Schrödinger equation and the Peierls-Nabarro barrier. Nonlinearity, 29:27, 2016.

[148] P.G. Kevrekidis, K.Ø. Rasmussen, and A.R. Bishop. Two-dimensional discrete breathers: Construction, stability, and bifurcations. Physical Review E, 61:2006, 2000.

[149] P.G. Kevrekidis, K.Ø. Rasmussen, and A.R. Bishop. Comparison of onedimensional and two-dimensional discrete breathers. Mathematics and Computers in Simulation, 55:449, 2001.

[150] P.G. Kevrekidis, K.Ø. Rasmussen, and A.R. Bishop. The discrete nonlinear Schrödinger equation: a survey of recent results. International Journal of Modern Physics B, 15:2833, 2001.

[151] S. Flach, K. Kladko, and R.S. MacKay. Energy Tresholds for Discrete Breathers in One- , Two- , and Three-Dimensional Lattices. Physical Review Letters, 78:1207, 1997.

[152] M.I. Weinstein. Excitation thresholds for nonlinear localized modes on lattices. Nonlinearity, 12:673, 1999.

[153] O. Bang and P.D. Miller. Exploiting discreteness for switching in arrays of nonlinear waveguides. Physica Scripta T, 67:26, 1996. 
[154] J.C. Eilbeck. Numerical Simulations of the Dynamics of Polypeptide Chains and Proteins. In C Kawabata and A.R. Bishop, editors, Computer Analysis for Life Science - Progress and Challenges in Biological and Synthetic Polymer Research, page 12. Tokyo, 1986.

[155] Y.S. Kivshar and D.K. Campbell. Peierls-Nabarro potential barrier for highly localized nonlinear modes. Physical Review E, 48:3077, 1993.

[156] J. Gómez-Gardeñes, F. Falo, and L.M. Floría. Mobile localization in nonlinear Schrödinger lattices. Physics Letters A, 332:213, 2004.

[157] S. Aubry and T. Cretegny. Mobility and reactivity of discrete breathers. Physica D, 119:34, 1998.

[158] M. Öster. Stability and Mobility of Localized and Extended Excitations in Nonlinear Schrödinger Models. PhD thesis, Linköping University, 2007.

[159] J. Cuevas, G. James, P.G. Kevrekidis, and K.J.H. Law. Vortex solutions of the discrete Gross-Pitaevskii equation starting from the anti-continuum limit. Physica D, 238:1422, 2009.

[160] K.J.H. Law, P.G. Kevrekidis, V. Koukouloyannis, I. Kourakis, D.J. Frantzeskakis, and A.R. Bishop. Discrete solitons and vortices in hexagonal and honeycomb lattices: Existence, stability, and dynamics. Physical Review E, 78:066610, 2008.

[161] K.J.H. Law, A. Saxena, P.G. Kevrekidis, and A.R. Bishop. Localized structures in kagome lattices. Physical Review A, 79:053818, 2009.

[162] L. Casetti and V. Penna. Vortex structures in a chain of coupled bosonic wells and the Mott regime. Journal of Low Temperature Physics, 126:455, 2002.

[163] N. Lagerquist. Discrete vortex switching in nonlinear optical waveguides. Bachelor thesis, Linköping University, 2013.

[164] J.C. Eilbeck. Nonlinear vibrational modes in a hexagonal molecule. In A. Davydov, editor, Physics of Many-Particle Systems, volume 12, page 41. Naukova Dumka, Kiev, 1987.

[165] L. Bernstein, J.C. Eilbeck, and A.C. Scott. The quantum theory of local modes in a coupled system of nonlinear oscillators. Nonlinearity, 3:293, 1990.

[166] L.J. Bernstein. Quantizing a self-trapping transition. Physica D, 68:174, 1993.

[167] A.C. Scott, J.C. Eilbeck, and H. Gilhøj. Quantum lattice solitons. Physica $D, 78: 194,1994$. 
[168] V.M. Agranovich. Biphonons and Fermi Resonance in Vibrational Spectra of Crystals. In V.M. Agranovich and R.M. Hochstrasser, editors, Spectroscopy and Excitation Dynamics of Condensed Molecular Systems, chapter 3, page 83. North-Holland, Amsterdam, 1983.

[169] A. Nenciu and G. Nenciu. Existence of exponentially localized Wannier functions for nonperiodic systems. Physical Review B, 47:112, 1993.

[170] W. Kohn. Analytic properties of Bloch waves and Wannier functions. Physical Review, 115:809, 1959.

[171] T. Müller, S. Fölling, A. Widera, and I. Bloch. State Preparation and Dynamics of Ultracold Atoms in Higher Lattice Orbitals. Physical Review Letters, 99:200405, 2007.

[172] G. Wirth, M. Ölschläger, and A. Hemmerich. Evidence for orbital superfluidity in the P-band of a bipartite optical square lattice. Nature Physics, $7: 147,2011$.

[173] M. Ölschläger, G. Wirth, and A. Hemmerich. Unconventional Superfluid Order in the F Band of a Bipartite Optical Square Lattice. Physical Review Letters, 106:015302, 2011.

[174] A. Eckardt, P. Hauke, P. Soltan-Panahi, C. Becker, K. Sengstock, and M. Lewenstein. Frustrated quantum antiferromagnetism with ultracold bosons in a triangular lattice. Europhysics Letters, 89:10010, 2010.

[175] P. Buonsante, V. Penna, and A. Vezzani. Attractive ultracold bosons in a necklace optical lattice. Physical Review A, 72:043620, 2005.

[176] O. Dutta, M. Gajda, P. Hauke, M. Lewenstein, D.-S. Lühmann, B.A. Malomed, T. Sowiński, and J. Zakrzewski. Non-standard Hubbard models in optical lattices: a review. Reports on Progress in Physics, 78:066001, 2015.

[177] G. Mazzarella, S.M. Giampaolo, and F. Illuminati. Extended Bose Hubbard model of interacting bosonic atoms in optical lattices: From superfluidity to density waves. Physical Review A, 73:013625, 2006.

[178] M. Eckholt and J. García-Ripoll. Pair condensation of bosonic atoms induced by optical lattices. Physical Review A, 77:063603, 2008.

[179] J.-Q. Liang, J.-L. Liu, W.-D. Li, and Z.-J. Li. Atom-pair tunneling and quantum phase transition in the strong-interaction regime. Physical Review A, 79:033617, 2009.

[180] Y.-M. Wang and J.-Q. Liang. Repulsive bound-atom pairs in an optical lattice with two-body interaction of nearest neighbors. Physical Review A, 81:045601, 2010.

[181] X.-F. Zhou, Y.-S. Zhang, and G.-C. Guo. Pair tunneling of bosonic atoms in an optical lattice. Physical Review A, 80:013605, 2009. 
[182] P.-I. Schneider, S. Grishkevich, and A. Saenz. Ab initio determination of Bose-Hubbard parameters for two ultracold atoms in an optical lattice using a three-well potential. Physical Review A, 80:013404, 2009.

[183] S. Trotzky, P. Cheinet, S. Fölling, M. Feld, U. Schnorrberger, A.M. Rey, A. Polkovnikov, E.A Demler, M.D. Lukin, and I. Bloch. Time-resolved observation and control of superexchange interactions with ultracold atoms in optical lattices. Science, 319:295, 2008.

[184] D.-S. Lühmann, O. Jürgensen, and K. Sengstock. Multi-orbital and densityinduced tunneling of bosons in optical lattices. New Journal of Physics, 14:033021, 2012.

[185] U. Bissbort, F. Deuretzbacher, and W. Hofstetter. Effective multibodyinduced tunneling and interactions in the Bose-Hubbard model of the lowest dressed band of an optical lattice. Physical Review A, 86:023617, 2012.

[186] T. Sowiński, O. Dutta, P. Hauke, L. Tagliacozzo, and M. Lewenstein. Dipolar Molecules in Optical Lattices. Physical Review Letters, 108:115301, 2012.

[187] G.G. Batrouni, F. Hébert, and R.T. Scalettar. Supersolid Phases in the OneDimensional Extended Soft-Core Bosonic Hubbard Model. Physical Review Letters, 97:087209, 2006.

[188] D. Rossini and R. Fazio. Phase diagram of the extended Bose-Hubbard model. New Journal of Physics, 14:065012, 2012.

[189] V. Scarola and S. Das Sarma. Quantum Phases of the Extended BoseHubbard Hamiltonian: Possibility of a Supersolid State of Cold Atoms in Optical Lattices. Physical Review Letters, 95:033003, 2005.

[190] P. Sengupta, L.P. Pryadko, F. Alet, M. Troyer, and G. Schmid. Supersolids versus Phase Separation in Two-Dimensional Lattice Bosons. Physical Review Letters, 94:207202, 2005.

[191] O. Jürgensen, F. Meinert, M.J. Mark, H.-C. Nägerl, and D.-S. Lühmann. Observation of Density-Induced Tunneling. Physical Review Letters, 113:193003, 2014.

[192] F. Meinert, M.J. Mark, K. Lauber, A.J. Daley, and H.-C. Nägerl. Floquet Engineering of Correlated Tunneling in the Bose-Hubbard Model with Ultracold Atoms. Physical Review Letters, 116:205301, 2016.

[193] A. Isacsson and S.M. Girvin. Multiflavor bosonic Hubbard models in the first excited Bloch band of an optical lattice. Physical Review A, 72:053604, 2005.

[194] F. Pinheiro, J.-P. Martikainen, and J. Larson. Confined p-band BoseEinstein condensates. Physical Review A, 85:033638, 2012. 
[195] E. Schrödinger. Der stetige Übergang von der Mikro- zur Makromechanik. Naturwissenschaften, 14:664, 1926.

[196] W.-M. Zhang, D.H. Feng, and R. Gilmore. Coherent states: Theory and some applications. Reviews of Modern Physics, 62:867, 1990.

[197] R.J. Glauber. Coherent and Incoherent States of the Radiation Field. Physical Review, 131:2766, 1963.

[198] E. Wright, J.C. Eilbeck, M.H. Hays, P.D. Miller, and A.C. Scott. The quantum discrete self-trapping equation in the Hartree approximation. Physica D, 69:18, 1993 .

[199] L. Amico and V. Penna. Dynamical Mean Field Theory of the Bose-Hubbard Model. Physical Review Letters, 80:2189, 1998.

[200] D. Ellinas, M. Johansson, and P.L. Christiansen. Quantum nonlinear lattices and coherent state vectors. Physica D, 134:126, 1999.

[201] P. Jason and M. Johansson. Quantum dynamics of lattice states with compact support in an extended Bose-Hubbard model. Physical Review A, 88:033605, 2013.

[202] R.A. Pinto and S. Flach. Quantum discrete breathers. In S. Keshavamurthy and P. Schlagheck, editors, Dynamical Tunneling: Theory and Experiment, chapter 14, page 385. CRC Press, Boca Raton, FL, 2011.

[203] J. Javanainen and U. Shrestha. Nonlinear phenomenology from quantum mechanics: soliton in a lattice. Physical review letters, 101:170405, 2008.

[204] W.Z. Wang, J.T. Gammel, A.R. Bishop, and M.I. Salkola. Quantum Breathers in a Nonlinear Lattice. Physical Review Letters, 76:3598, 1996.

[205] M.W. Jack and M. Yamashita. Bose-Hubbard model with attractive interactions. Physical Review A, 71:023610, 2005.

[206] V. Fleurov. Discrete quantum breathers: what do we know about them? Chaos, 13:676, 2003.

[207] J.I. Cirac, M. Lewenstein, K. Mølmer, and P. Zoller. Quantum superposition states of Bose-Einstein condensates. Physical Review A, 57:1208, 1998.

[208] T.-L. Ho and C.V. Ciobanu. The Schrödinger cat family in attractive Bose gases. Journal of Low Temperature Physics, 135:257, 2004.

[209] S. Aubry, S. Flach, K. Kladko, and E. Olbrich. Manifestation of Classical Bifurcation in the Spectrum of the Integrable Quantum Dimer. Physical Review Letters, 76:1607, 1996.

[210] P. Buonsante, V. Penna, and A. Vezzani. Quantum signatures of the self-trapping transition in attractive lattice bosons. Physical Review A, 82:043615, 2010. 
[211] E. Altman, A. Polkovnikov, E. Demler, B.I. Halperin, and M.D. Lukin. Superfluid-Insulator Transition in a Moving System of Interacting Bosons. Physical Review Letters, 95:020402, 2005.

[212] A. Polkovnikov, E. Altman, E. Demler, B. Halperin, and M.D. Lukin. Decay of superfluid currents in a moving system of strongly interacting bosons. Physical Review A, 71:063613, 2005.

[213] M.M. Nieto. Quantum Phase and Quantum Phase Operators: Some Physics and Some History. Physica Scripta T, 48:5, 1993.

[214] P. Carruthers and M.M. Nieto. Phase and Angle Variables in Quantum Mechanics. Reviews of Modern Physics, 40:411, 1968.

[215] C. Lee, T.J. Alexander, and Y.S. Kivshar. Melting of Discrete Vortices via Quantum Fluctuations. Physical Review Letters, 97:180408, 2006.

[216] G. Arwas, A. Vardi, and D. Cohen. Superfluidity and Chaos in low dimensional circuits. Scientific reports, 5:13433, 2015.

[217] W. Zwerger. Mott-Hubbard transition of cold atoms in optical lattices. Journal of Optics B: Quantum and Semiclassical Optics, 5:S9, 2003.

[218] M.P.A. Fisher, P.B. Weichman, G. Grinstein, and D.S. Fisher. Boson localization and the superfluid-insulator transition. Physical Review B, 40:546, 1989.

[219] D. Jaksch, C. Bruder, J.I. Cirac, C.W. Gardiner, and P. Zoller. Cold Bosonic Atoms in Optical Lattices. Physical Review Letters, 81:3108, 1998.

[220] T. Stöferle, H. Moritz, C. Schori, M. Köhl, and T. Esslinger. Transition from a Strongly Interacting 1D Superfluid to a Mott Insulator. Physical Review Letters, 92:130403, 2004.

[221] M. Köhl, H. Moritz, T. Stöferle, C. Schori, and T. Esslinger. Superfluid to Mott insulator transition in one, two, and three dimensions. Journal of Low Temperature Physics, 138:635, 2005. 


\section{List of included Publications}

[I] Exact localized eigenstates for an extended Bose-Hubbard model with pair-correlated hopping

Jason P, Johansson M

Physical Review A 85, 011603(R) (2012)

[II] Quantum signatures of an oscillatory instability in the BoseHubbard trimer

Jason P, Johansson M, Kirr K

Physical Review E 86, 016214 (2012)

[III Quantum dynamics of lattice states with compact support in an extended Bose-Hubbard model

Jason P, Johansson M

Physical Review A 88, 033605 (2013)

[IV] Charge flipping vortices in the discrete nonlinear Schrödinger trimer and hexamer

Jason P, Johansson M

Physical Review E 91, 022910 (2015)

[V] Discrete breathers for a discrete nonlinear Schrödinger ring coupled to a central site

Jason P, Johansson M

Physical Review E 93, 012219 (2016)

[VI] Quantum signatures of charge flipping vortices in the BoseHubbard trimer

Jason P, Johansson M

Submitted to Physical Review E 


\section{My contribution to the papers}

[1] Exact localized eigenstates for an extended Bose-Hubbard model with pair-correlated hopping

P. Jason M. Johansson Physical Review A 85, 011603(R) (2012)

This paper is based on the work I did in my Master thesis. I took part in planning the project and performed all calculations. I wrote parts of the draft to the paper.

[2] Quantum signatures of an oscillatory instability in the Bose-Hubbard trimer

P. Jason M. Johansson K. KIRR Physical Review E 86, 016214 (2012)

I took part in planning and executing the project, and all calculations presented in the paper were performed by me. This work was initiated by Johansson and Kirr prior to my involvement, and some calculations were originally performed by Kirr in the initial stage of the project, which I later reproduced. I wrote parts of the draft to the paper.

[3] Quantum dynamics of lattice states with compact support in an extended Bose-Hubbard model

P. Jason M. Johansson Physical Review A 88, 033605 (2013)

I took part in planning and executing the project, and all calculations presented in the paper were performed by me. I wrote the majority of the manuscript.

[4] Charge flipping vortices in the discrete nonlinear Schrödinger trimer and hexamer

P. Jason M. Johansson Physical Review E 91, 022910 (2015)

This work is based on the Bachelor thesis by Nicklas Lagerquist. I took part in planning and executing the project, and all calculations presented in the paper were performed by me. I wrote the majority of the manuscript.

[5] Discrete breathers for a discrete nonlinear Schrödinger ring coupled to a central site

P. Jason M. Johansson Physical Review E 93, 012219 (2016)

This work was initiated by me, and I took part in planning and executing the project. All calculations presented in the paper were performed by me. I wrote the majority of the manuscript.

[6] Quantum signatures of charge flipping vortices in the Bose-Hubbard trimer P. Jason M. Johansson Submitted to Physical Review E

I took part in planning and executing the project, and all calculations presented in the paper were performed by me. I wrote the majority of the manuscript. 


\section{Related, not included Publications}

[I] Quantum Compactons in an extended Bose-Hubbard model P. Jason

Master Thesis, Linköping University (2011)

[II] Comparisons between classical and quantum mechanical nonlinear lattice models

P. Jason

Licentiate Thesis, Linköping University (2014)

[III] Breather Mobility and the Peierls-Nabarro Potential: Brief Review and Recent Progress

M. Johansson and P. Jason

J.F.R. Archilla, N. Jiménez, V.J. Sánchez-Morcillo, L.M. García-Raffi (eds.) Quodons in Mica: Nonlinear Localized Travelling Excitations in Crystals. Chapter 6. Springer, Cham (2015) 


\section{Included Publications}

The articles associated with this thesis have been removed for copyright reasons. For more details about these see:

http://urn.kb.se/resolve?urn=urn:nbn:se:liu:diva-129564 